\title{
Statistical analysis of synaptic transmission at the calyx of Held synapse
}

\author{
Dissertation \\ zur Erlangung des Doktorgrades \\ der Mathematisch-Naturwissenschaftlichen Fakultäten \\ der Georg-August-Universität Göttingen
}

vorgelegt von

Volker Scheuß

aus Hamburg

Göttingen, den 18. September 2000 
D7

Referent: Prof. Dr. E. Neher

Korreferent: Prof. Dr. T. Geisel

Tag der mündlichen Prüfung: 2. November 2000 


\section{Contents}

1. Introduction 3

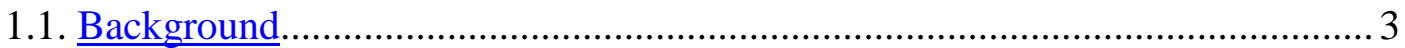

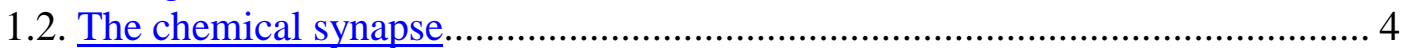

1.3. Mechanisms underlying synaptic short-term plasticity................................... 6

1.4. Quantal analysis and statistical models of synaptic transmission.......................6

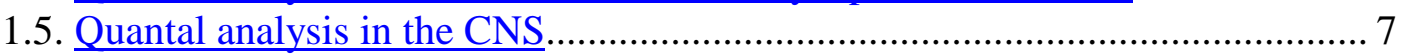

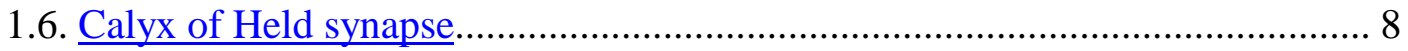

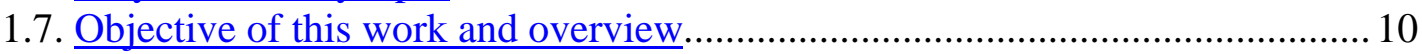

2. Theory and Methods 11

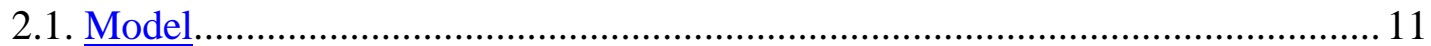

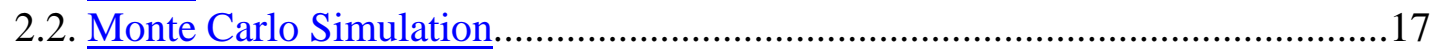

2.2.1 Pool kinetics $\quad 18$

2.2.2 Evoked release simulation and probability distributions $\quad 23$

2.2.3 Quantal size assignement $\quad 25$

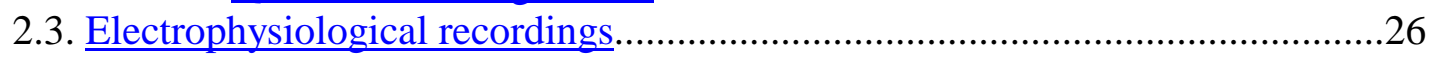

2.3.1 Solutions $\quad 27$

2.3.2 Dissection and slice preparation $\quad 28$

$\begin{array}{ll}2.3 .3 \text { Electrophysiology } & 29\end{array}$

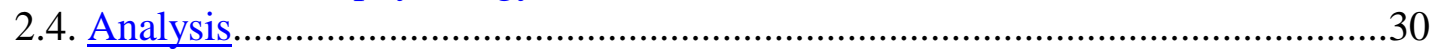

3. Results 33

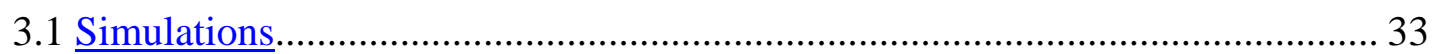

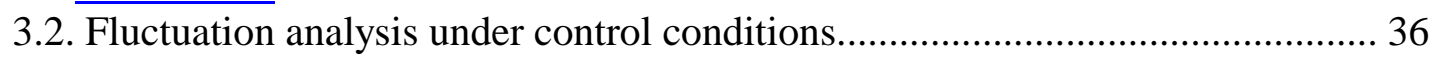

3.2.1. Variance-mean analysis under control conditions 36

$\begin{array}{ll}\text { 3.2.2. Covariance analysis under control conditions } & 38\end{array}$

3.3. Fluctuation analysis in the presence of CTZ............................................... 40

3.3.1. Variance-mean analysis in the presence of CTZ 40

3.3.2. Covariance analysis in the presence of CTZ 42

3.4. Fluctuation analysis in the presence of CTZ and Kyn................................... 44

3.4.1. Variance-mean analysis in the presence of CTZ and Kyn 44

3.4.2. Covariance analysis in the presence of CTZ and Kyn 47

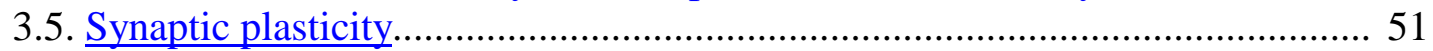

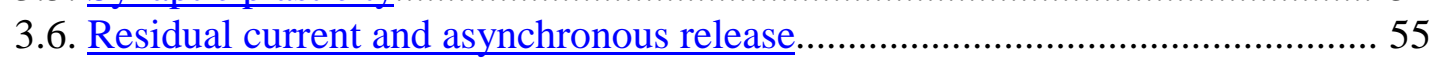

3.7. Cumulative EPSC amplitudes.................................................................. 56

3.8. Stationary and non-stationary noise analysis combined................................... 58 
4.1. The method.

4.2. Application of the method and summary of the results....................................63

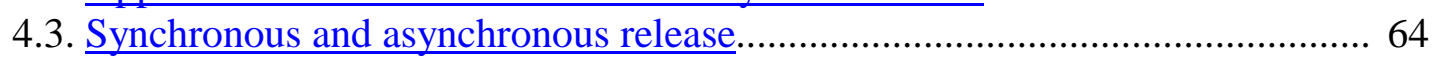

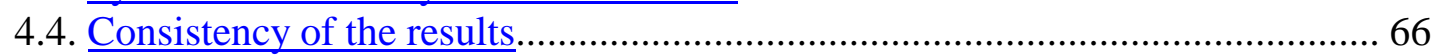

4.5. Comparison of the reduction in quantal size to other studies............................69 69

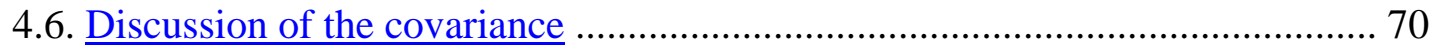

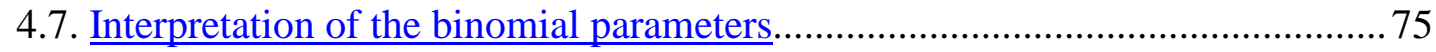

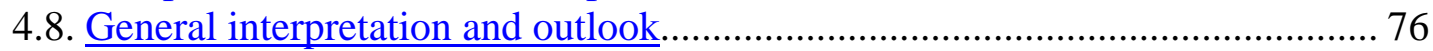

$\begin{array}{lr}\text { Appendix } & 79\end{array}$

A. Non-stationary noise analysis in the presence of intra- and intersite quantal variability

B. Parameters applied in the simulations

C. Calculation of the uncertainty in the segmented estimation of the variance

D. Calculation of the uncertainty in the segmented estimation of the covariance

E. Noise-analysis in the presence of saturation and desensitisation of postsynaptic receptors

$\underline{\text { References }}$

Acknowledgements

$\underline{\text { Publications }}$

$\underline{\text { Curriculum Vitae }}$ 


\section{Introduction}

\subsection{Background}

Signal transmission between neurons takes place at regions of mutual contact, which are specialised for this purpose. They were termed "synapses" by Sir Charles Sherrington derived from the Greek "to fasten together" (Foster and Sherrington 1897). Two types of synapses are known. According to the mechanism of signal transmission they are termed chemical and electrical synapses. The majority of synapses in any mammalian nervous system are of the chemical synapse type. Transmission via chemical synapses is not static in the respect that equal inputs to the presynaptic terminal cause invariantly equal outputs at the postsynapse. Instead the size of the postsynaptic response depends on the previous history of presynaptic activity. It can be only a fraction or many times the size of an isolated postsynaptic response. This phenomenon is referred to as synaptic plasticity. Different modes of synaptic plasticity are distinguished according to the time scales of establishment and persistence of the modulation, and whether it is manifested as an increase or decrease of the response size (for reviews see Zucker 1989, Zucker 1999). Short-term synaptic plasticity comprises modulations occurring within a few milliseconds up to tens of seconds. Modulations on these time scales represent the dynamic properties of the synaptic machinery and influences the information processing function of neuronal circuits. Studies regarding the molecular constituents of the synaptic machinery often introduce specific artificial or physiological modifications and assay the functional consequences by their effect on the synaptic transmission properties. The classical way to quantify such effects is to carry out a quantal analysis, originally introduced by Sir Bernhard Katz (Del Castillo and Katz 1954) for synaptic transmission at the neuromuscular junction (nmj). Since then this method has been improved and also applied to synaptic transmission in the central nervous system (CNS). However, an alternative approach referred to as ensemble noise analysis (Clamann et al. 1989), multiple - probability - fluctuation analysis (Silver et al. 1998) or variance - mean analysis (Reid and Clements 1999), has some important advantages. It has emerged from ion channel analysis (Sigworth 1980) and was adopted for the analysis of 
synaptic transmission over the past decade. In this project this method was further modified and extended for analysing the mechanisms underlying synaptic plasticity in more detail, and was applied to transmission at the calyx of Held synapse, a chemical synapse in the mammalian CNS, which displays strong use-dependent depression.

\subsection{The chemical synapse}

The synapse is principally subdivided into two parts separated by the synaptic cleft. The presynaptic terminal acts like a transmitter and the postsynaptic neuron as a receiver of chemical signals.

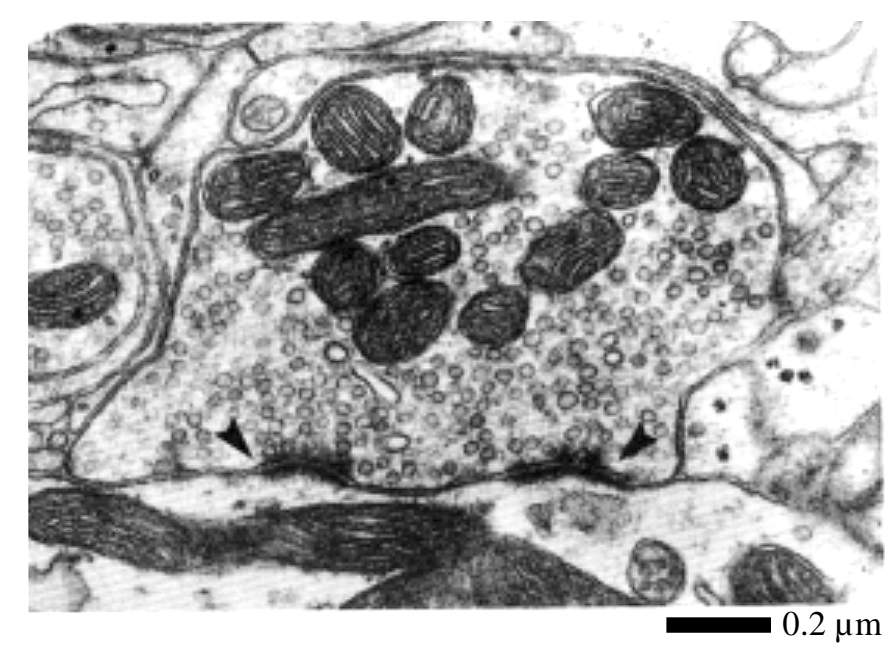

Fig. 1: Morphology of the synapse. Electron micrographs of synaptic connections show specialisations in form of coextensive electron dense bands in the opposing membranes of two neurons (arrows). In the presynapse the latter is called active zone and in the postsynapse postsynaptic density (PSD). Small synaptic vesicles are found docked to and clustered around the active zone.

Information about the morphology and composition of the synapse comes from electron microscopy (see Fig. 1) and biochemical studies (for reviews see Walmsley et al. 1998, Garner et al. 2000). Synapses contain one or more distinct specialisations, which consist of coextensive electron dense bands in the opposing presynaptic and postsynaptic plasma membranes. Membrane bound organelles, the neurotransmitter containing synaptic vesicles, are found to be docked to and clustered around the presynaptic density, called active zone. The active zone contains $\mathrm{Ca}^{2+}$-channels and the molecular machinery controlling fusion of vesicles with the plasma membrane. The postsynaptic density on the other hand contains neurotransmitter receptors and ligand-gated ion channels. 
Functionally the basic scheme of synaptic transmission is as follows (e.g. Alberts et al. 1994, and see Fig. 2A): Across the membrane of neurons there is a potential difference called membrane potential, which is in the range of 50 to $100 \mathrm{mV}$. Along the processes and within neurons, neuronal signals are carried by propagating depolarisations from the resting potential. Upon arrival of such an action potential (AP) at the presynaptic terminal, voltage-gated $\mathrm{Ca}^{2+}$-channels open. $\mathrm{Ca}^{2+}$ ions enter the terminal and bind to some sensor. This activates some molecular machinery,
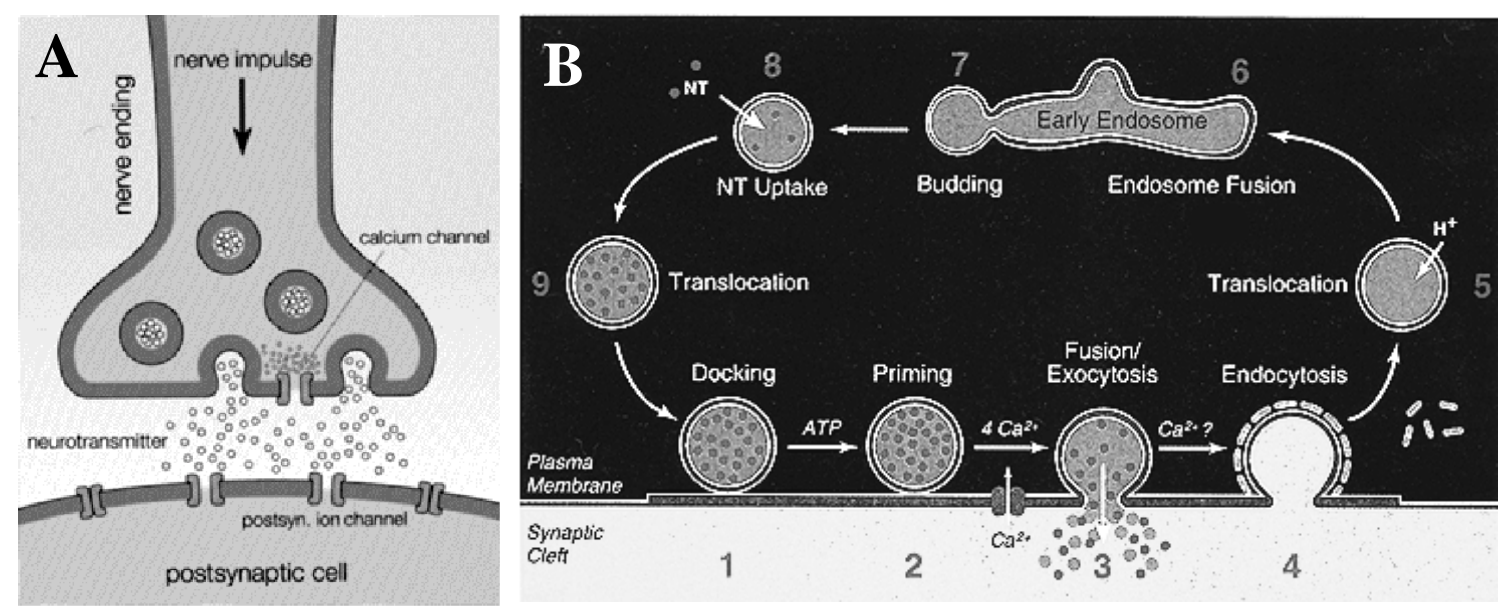

Fig. 2: Function of the synapse. (A) Scheme visualising the functional aspects of synaptic transmission. For details refer to the text. (B) Vesicle cycle as suggested by Südhof (1995).

which causes synaptic vesicles to fuse with the plasma membrane. By fusing with the plasma membrane synaptic vesicles release their neurotransmitter content into the synaptic cleft. After diffusion through the synaptic cleft, neurotransmitter molecules reach the postsynaptic membrane. Binding of neurotransmitter activates ionotropic receptors on the postsynaptic neuron, which causes ligand-gated ion channels to open. This results in a change of the postsynaptic membrane potential and thus finally converts the chemical signal again into an electrical signal. The probability of vesicle fusion is strongly dependent on the amount of $\mathrm{Ca}^{2+}$-influx into the terminal (for reviews see Neher 1998, Zucker 1999) and can be regulated by the applied extracellular calcium concentration $\left[\mathrm{Ca}^{2+}\right]_{\mathrm{ext}}$ in experiments. In the absence of stimulation, spontaneous synaptic activity is frequently observed, which is associated with the fusion of single vesicles, and gives rise to so called miniature or quantal responses. After fusion, vesicles are retrieved by endocytosis and recycled. The 
complete vesicle cycle consists of fusion (exocytosis), retrieval (endocytosis), translocation, refilling, storage, docking to the plasma membrane and priming to gain fusion competence again (see Fig. 2B). It is controlled by a complex molecular machinery. In agreement with this, pools of vesicles in different states were distinguished morphologically, functionally and biochemically (Garner et al. 2000, Voets et al. 1999, Südhof 1995).

\subsection{Mechanisms underlying synaptic short-term plasticity}

As introduced already above, synaptic short-term plasticity refers to modulations of the postsynaptic response size on a time scale from a few milliseconds to tens of seconds during ongoing presynaptic activity. Multiple cellular mechanisms, most likely acting simultaneously in parallel, could be responsible for short-term plasticity (for reviews see Zucker 1989, Zucker 1999). Established or proposed mechanisms, which cause facilitation or an increase in response size, are build-up of residual $\mathrm{Ca}^{2+}$ in the synaptic terminal (for reviews see Kasai 1993, Neher 1998) and transient facilitation of the presynaptic $\mathrm{Ca}^{2+}$-conductance (Cuttle et al. 1998, Borst and Sakmann 1998). For depression or a reduction of the response size the established and proposed mechanisms are depletion of the available store of release competent vesicles (Hurlbut et al. 1990, Stevens and Tsujimoto 1995), presynaptic $\mathrm{Ca}^{2+}$-current inactivation (Wu and Saggau 1997, Forsythe et al. 1998), adaption of the $\mathrm{Ca}^{2+}$-sensor for exocytosis (Hsu et al. 1996), autoreceptor activation (von Gersdorff et al. 1997), incomplete refilling with neurotransmitter of fast recycling vesicles (Behrends and Rumpel 2000) and postsynaptic receptor desensitisation (Trussel at al. 1993, Otis et al. 1996a). From the relative dominance of several mechanisms, the whole range of properties found in different synaptic connections should arise (Dittman et al. 2000).

\subsection{Quantal analysis and statistical models of synaptic transmission}

The quantal theory was originally introduced by Del Castillo and Katz (1954) based on the observation that postsynaptic responses in a muscle fibre vary randomly between integer multiples of the spontaneous miniature response. Quantal analysis is based on binomial statistics, including Poisson statistics as a limiting case, with three parameters determining the size of a stimulus-evoked response: The average size of the quantal unit $\mathrm{q}$, the number of sites $\mathrm{N}$ that can release a quantum, and the 
probability of release $\mathrm{p}$ in response to stimulation at each release site (for a review see McLachlan 1978). This set of parameters is readily evident from the morphology and function of synapses as discussed above, and the aim of quantal analysis is to correlate any modification of transmission with a change in these parameters. The original analytical approach of quantal analysis is to record as many evoked postsynaptic currents (EPSCs) or potentials (EPSPs) as possible under constant conditions. The EPSC or EPSP data is summarised in an amplitude histogram and this is treated as a multimodal distribution in which each mode represents a different number of quanta released. In practice, this requires the identification of peaks in the histogram spaced a quantal unit apart, which as a control can be compared to the amplitude of spontaneously occurring miniature currents (mEPSCs) or potentials (mEPSPs) (for reviews see Redman 1990, Walmsley 1993). This approach was very successfully applied to transmission at the nmj. In the CNS however, it proofed to be complicated and therefore controversial, because of structural and functional factors not encountered at the nmj to such an extend (Redman 1990, Walmsley 1993).

\subsection{Quantal analysis in the CNS}

Since central neurons receive large numbers of synaptic inputs, there is usually uncertainty regarding the number of inputs activated and the origin of spontaneous unitary events is not known. Synaptic currents suffer variable amounts of electrotonic attenuation due to different somato-dendritic locations of the synaptic contacts and are often small compared to the background noise, especially miniature responses. If spontaneous synaptic events (minis) can be recorded, broad amplitude distributions are obtained with coefficients of variation $(\mathrm{CV})$ in the range of $44 \%$ to $93 \%$ in different preparations (Frerking and Wilson 1996). Quantal variability can result from electrotonic attenuation, variable neurotransmitter content or size of vesicles, variability in the size of the PSD or in the postsynaptic receptor density, postsynaptic channel noise and the presence of different conductance states (reviewed in von Kitzing et al. 1994). Furthermore, considerable heterogeneity in the release probability at different release sites is present at certain synaptic connections (Rosenmund et al. 1992, Murthy et al. 1997). These findings can be accounted for by applying compound binomial, multinomial and compound multinomial models (Redman 1990, Walmsley 1993, Silver et al. 1998). However, in case of histograms simply lacking peaks, quantisation is contentious. The alternative approach called 
ensemble noise analysis, multiple-probability-fluctuation analysis or simply variancemean analysis, emerged from the fluctuation analysis of ion-channels (Sigworth 1980), and was adopted to synaptic transmission (Clamann et al. 1989, Frerking and Wilson 1996, Silver et al. 1998, Reid and Clements 1999, Oleskevich et al. 2000, Clements and Silver 2000). Mean and variance of stimulus-evoked responses are computed directly and model independent under different condition (e.g. different $\left.\left[\mathrm{Ca}^{2+}\right]_{\mathrm{ext}}\right)$ thereby varying the release probability and consequently the mean response amplitude. The relationship between variance and mean is compared to the prediction by one of the above mentioned models and the model parameters are determined. This approach has the advantage that the properties of transmission under different conditions are simultaneously analysed, which provides more, and more reliable information. Furthermore the extension of the simple binomial model to account for the above mentioned complexities are integrated in a more intuitive fashion than in the classical histogram method.

\subsection{Calyx of Held synapse}

The calyx of Held synapse is one of two giant synapses in the auditory pathway, both named after Held (1893). It is located in the medial nucleus of the trapezoid body (MNTB), which is part of a binaural pathway concerned with localisation of a sound source by interaural intensity differences (Gummer and Zenner 1996). This synapse arises from a single presynaptic axon and forms a single large calyceal terminal onto the soma of a principal neurone in the MNTB. Each of these neurons receives only a single giant synapse (Forsythe and Barnes-Davies 1993). The neurotransmitter released by the terminal is glutamate and generates dual component postsynaptic currents. The fast component results from activation of AMPA-type glutamate receptors, while the slow component is generated by activation of NMDA-type glutamate receptors, which are distinguished by their specific artificial agonists (activators) amino-3-hydroxy-5-methyl-4-isoxazole-proprionic acid (AMPA) and Nmethyl-D-aspartate (NMDA) (Forsythe et al. 1995).

The calyx of Held synapse preparation provides critical prerequisites as demanded for a proper quantal or noise analysis (Korn and Faber 1991, Walmsley 1993, Forsythe et al. 1995). Each postsynaptic cell receives only a single defined input. The synapses form on the somata of the neurons in the MNTB, which have a sparse dendritic tree (Banks and Smith 1992). This configuration is ideal for voltage clamping. 
Furthermore it allows the detection and recording of spontaneous synaptic activity in form of mEPSCs (Borst and Sakmann 1996, Chuma and Ohmori 1998).

Regarding short-term synaptic plasticity, the calyx of Held synapse displays pronounced synaptic depression upon repetitive stimulation at physiological $\left[\mathrm{Ca}^{2+}\right]_{\mathrm{ext}}$ or above, while transient facilitation is observed at reduced $\left[\mathrm{Ca}^{2+}\right]_{\mathrm{ext}}$ (Borst et al 1995, von Gersdorff et al. 1997, Schneggenburger et al. 1999). A major contribution to depression at the calyx of Held synapse is assumed to arise from postsynaptic receptor desensitisation (Borst et al. 1995, Geiger et al. 1995) and vesicle pool depletion (von Gersdorff et al 1997). Minor mechanisms contributing to depression are the activation of metabotropic autoreceptors for glutamate (mGluR) in the presynaptic terminal (von Gersdorff et al 1997). Their activation was shown to suppress a $\mathrm{Ca}^{2+}$ conductance of the presynaptic terminal (Takahashi et al. 1996). The presynaptic calcium current was shown to inactivate with slow recovery (Forsythe et al. 1998) after being transiently facilitated (Cuttle et al. 1998, Borst and Sakmann 1998) through a $\mathrm{Ca}^{2+}$-dependent mechanism. Furthermore activity dependent $\mathrm{Ca}^{2+}$ depletion from the synaptic cleft was reported (Borst and Sakmann 1999).

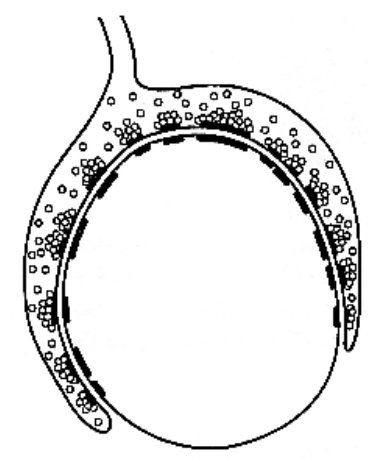

Fig. 3: Schematic drawing of a calyx-type somatic terminal. The circles depict synaptic vesicles and the strong lines synaptic specialisations (from Walmley et al. (1998)).

In comparison to other synapses in the CNS the calyx synapse is atypical. Typically the majority of synaptic contacts appear as small specialisations, called boutons, along the axon, and contain only a few active zones (Schikorski and Stevens 1997, Silver et al. 1998). However the calyx synapse might be regarded as a giant bouton with a vast 
number of active zones, which release into a laterally extensive synaptic cleft (Casey and Feldman 1988, Walmsley et al. 1998), see Fig. 3.

\subsection{Objective of this work and overview}

The main goal of this work was to elucidate the mechanism underlying the strong usedependent depression of synaptic transmission at the Calyx of Held synapse (von Gersdorff et al. 1997, Schneggenburger et al. 1999). For this purpose non-stationary noise analysis of the EPSC amplitude fluctuation was applied and modified to account for possible correlation between the amplitudes of successive EPSCs in response to a train of stimuli. The theory and experimental methods applied are described in chapter 2. The modified analysis was tested on data generated by Monte Carlo simulations of synaptic transmission. The analysis was then applied to recorded data, which revealed a reduction in quantal size along the stimulus train, contributing significantly to the observed synaptic depression. The apparent quantal size reduction could be attributed to desensitisation and saturation of postsynaptic receptors by application of drugs abolishing these phenomena. This is documented in the first part of the chapter 3 . The second part considers experiments performed to test the implications of the observed phenomena and to obtain independent controls. In chapter 4 the work is summarised, the consistency between different experiments and with the literature is provided, and extensions of the analytical approach are introduced and discussed regarding the solution of remaining complications. 


\section{Theory and Methods}

\subsection{Model}

This model of neurotransmitter release is based on the binomial model developed by Quastel (1997). It was extended to account for quantal variation (Frerking and Wilson 1996) and changes in the average quantal size with repetitive activity. A general description of the statistical properties of stimulus-evoked release is derived by considering each release site individually before expanding to the whole population of sites simultaneously (Frerking and Wilson 1996). A release site is by definition not necessarily equivalent to a morphologically identified active zone. An individual release site responds to a stimulus in an all-or-none fashion. In case that release occurs, this generates a quantal postsynaptic response of size q. The probability that a release event occurs at a given site upon stimulation is the release probability p. After a release event, the release site is empty and reoccupied with a certain recovery time gaining release competence again (see Fig. 4A). In contrast to other similar studies (Clamann et al. 1989, Frerking and Wilson 1996, Silver et al. 1998, Reid and Clements 1999, Oleskevich 2000, Clements and Silver 2000), the release probability $p$ is considered here to be composed as the product of two probabilities (Zucker 1989, Quastel 1997): The probability $\mathrm{p}_{\mathrm{A}}(\mathrm{A}=$ availability), that a release site is occupied with a release-competent vesicle, and, in case it is occupied, the probability for release upon stimulation $\mathrm{p}_{0}(\mathrm{O}=$ output $)$, following the terminology by Quastel (1997). This allows to account for depletion of release competent sites during repetitive stimulation. The relations are here derived neglecting quantal variance for the sake of simplicity in the demonstration of the approach. In appendix A, the relations are derived considering intra- and inter-site quantal variance (Frerking and Wilson 1996). First, release events at a single release site are considered. The postsynaptic response to the $\mathrm{i}^{\text {th }}$ stimulus in a train of stimuli at a single release site is given by

$$
\mathrm{y}_{\mathrm{i}}=\mathrm{q}_{\mathrm{i}} \mathrm{x}_{\mathrm{i}} \quad \text { where } \quad \mathrm{x}_{\mathrm{i}}=\left\{\begin{array}{lc}
1 & \text { if vesicle fuses at stimulus } \mathrm{i} \\
0 & \text { otherwise }
\end{array}\right.
$$

with the compound probability for a release event $\mathrm{p}\left(\mathrm{x}_{\mathrm{i}}=1\right)=\mathrm{p}_{\mathrm{Oi}} \mathrm{p}_{\mathrm{Ai}}$ 
Thus in case a stimulus train is repeated under constant conditions the average amplitude of the postynaptic response to the $\mathrm{i}^{\text {th }}$ stimulus at a single release site is

$$
\bar{y}_{\mathrm{i}}=\mathrm{q}_{\mathrm{i}} \mathrm{p}_{\mathrm{Oi}} \mathrm{p}_{\mathrm{Ai}}
$$

The variance or autocovariance of the postynaptic response to the $\mathrm{i}^{\text {th }}$ stimulus at a single release site is

$$
\begin{aligned}
\operatorname{var}_{\mathrm{i}}= & \operatorname{cov}_{\mathrm{i}, \mathrm{i}}=<\mathrm{y}_{\mathrm{i}}^{2}>-<\mathrm{y}_{\mathrm{i}}>^{2}=<\mathrm{q}_{\mathrm{i}}^{2} \mathrm{x}_{\mathrm{i}}^{2}>-<\mathrm{q}_{\mathrm{i}} \mathrm{x}_{\mathrm{i}}>^{2} \\
& =\mathrm{q}_{\mathrm{i}}^{2} \mathrm{p}\left(\mathrm{x}_{\mathrm{i}}=1\right)-\mathrm{q}_{\mathrm{i}}^{2} \mathrm{p}\left(\mathrm{x}_{\mathrm{i}}=1\right)^{2} \\
& =\mathrm{q}_{\mathrm{i}}^{2} \mathrm{p}_{\mathrm{O} i} \mathrm{p}_{\mathrm{Ai}}\left(1-\mathrm{p}_{\mathrm{Oi}} \mathrm{p}_{\mathrm{Ai}}\right)
\end{aligned}
$$

The covariance between the amplitude of consecutive responses $i$ and $j$ in the train at a single site is

$$
\operatorname{cov}_{\mathrm{i}, \mathrm{j}}=<\mathrm{y}_{\mathrm{i}} \mathrm{y}_{\mathrm{j}}>-\overline{\mathrm{y}}_{\mathrm{i}} \overline{\mathrm{y}}_{\mathrm{j}}
$$

with eq. M3

$$
\begin{aligned}
& =<q_{i} x_{i} q_{j} x_{j}>-q_{i} q_{j} p_{O i} p_{A i} p_{O j} p_{A j} \\
& =<q_{i} q_{j}><x_{i} x_{j}>-q_{i} q_{j} p_{O i} p_{A i} p_{O j} p_{A j} \\
& \text { with } \quad<x_{i} x_{j}>=p\left(x_{j}=1, x_{i}=1\right) \\
& =p\left(x_{j}=1 \mid x_{i}=1\right) p\left(x_{i}=1\right) \\
& =p_{O j} p\left(z_{j}=1 \mid x_{i}=1\right) p_{O i} p_{A i}
\end{aligned}
$$


where

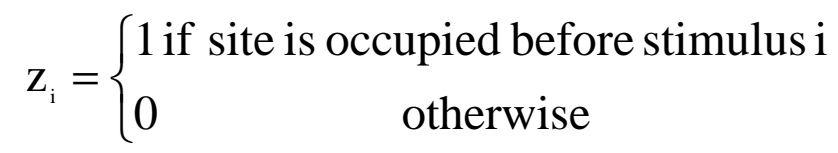

with

$$
\begin{aligned}
& \mathrm{p}\left(\mathrm{z}_{\mathrm{i}}=1\right)=\mathrm{p}_{\mathrm{Ai}} \\
& \mathrm{p}\left(\mathrm{z}_{\mathrm{j}}=1 \mid \mathrm{x}_{\mathrm{i}}=1\right)=\mathrm{p}_{\mathrm{Aj \textrm {j }}}
\end{aligned}
$$

where $\mathrm{p}_{\mathrm{Aj} \mathrm{j}}$ is the conditional probability that site is occupied at stimulus $\mathrm{j}$ given it was released at stimulus i. Therefore

$$
\begin{aligned}
\operatorname{cov}_{\mathrm{i}, \mathrm{j}} & =<\mathrm{q}_{\mathrm{i}} \mathrm{q}_{\mathrm{j}}>\mathrm{p}_{\mathrm{Oi}} \mathrm{p}_{\mathrm{Ai}} \mathrm{p}_{\mathrm{O} \mathrm{j}} \mathrm{p}_{\mathrm{Aj \textrm {j }}}-\mathrm{q}_{\mathrm{i}} \mathrm{q}_{\mathrm{j}} \mathrm{p}_{\mathrm{Oi}} \mathrm{p}_{\mathrm{Ai}} \mathrm{p}_{\mathrm{Oj}} \mathrm{p}_{\mathrm{Aj}} \\
& =\mathrm{p}_{\mathrm{Oi}} \mathrm{p}_{\mathrm{Ai}} \mathrm{p}_{\mathrm{O} j}\left(<\mathrm{q}_{\mathrm{i}} \mathrm{q}_{\mathrm{j}}>\mathrm{p}_{\mathrm{Aj \textrm {j }}}-\mathrm{q}_{\mathrm{i}} \mathrm{q}_{\mathrm{j}} \mathrm{p}_{\mathrm{Aj}}\right)
\end{aligned}
$$

It is very unlikely that there is a correlation between the size among neurotransmitter quanta released from the same site, i.e. $\left\langle\mathrm{q}_{\mathrm{i}} \mathrm{q}_{\mathrm{j}}\right\rangle=\mathrm{q}_{\mathrm{i}} \mathrm{q}_{\mathrm{j}}$. However, there could be a correlation in the postsynaptic quantal responses for example in case of postsynaptic receptor desensitisation. The implications of such a case are considered in the discussion, but in the following calculations this is neglected. The covariance between the amplitude of consecutive responses $\mathrm{i}$ and $\mathrm{j}$ in the train at a single site is therefore

$$
\operatorname{cov}_{\mathrm{i}, \mathrm{j}}=\mathrm{p}_{\mathrm{Oi}} \mathrm{p}_{\mathrm{Ai}} \mathrm{p}_{\mathrm{O} \mathrm{j}}\left(\mathrm{p}_{\mathrm{A} j \mathrm{i}}-\mathrm{p}_{\mathrm{Aj}}\right) \mathrm{q}_{\mathrm{i}} \mathrm{q}_{\mathrm{j}}
$$

For the general description of the statistical properties of stimulus-evoked release, the properties of a single release site as derived above are now expanded to a population of $\mathrm{N}$ release sites. Under the assumptions that release sites are statistically independent and released quanta add linearly, the statistics of the population is given as the sum of the statistics of the individual sites. In addition, the absence of any heterogeneity is assumed, i.e. all sites have the same release probability $p_{o}$, the same 
average occupancy probability $\mathrm{p}_{\mathrm{A}}$, and the same uniform quantal size $\mathrm{q}$. In this case the terms contributed by each release site are equal, and the statistical parameters of the population of $\mathrm{N}$ sites are $\mathrm{N}$ times the parameter for an individual site:

The average overall response amplitude at the $\mathrm{i}^{\text {th }}$ stimulus is then the sum of eq. M3 over all sites

$$
\bar{Y}_{i}=N \bar{y}_{i}=N q_{i} p_{o i} p_{A i}
$$

The overall variance or autocovariance of the postynaptic response to the $\mathrm{i}^{\text {th }}$ stimulus is from eq. M4

$$
\operatorname{Var}_{\mathrm{i}}=\mathrm{N} \operatorname{var}_{\mathrm{i}}=\mathrm{Nq}_{\mathrm{i}}^{2} \mathrm{p}_{\mathrm{oi}} \mathrm{p}_{\mathrm{Ai}}\left(1-\mathrm{p}_{\mathrm{Oi}} \mathrm{p}_{\mathrm{Ai}}\right)
$$

The overall covariance between the amplitudes at stimulus $i$ and $j$ is from eq. M8

$$
\operatorname{Cov}_{\mathrm{i}, \mathrm{j}}=\mathrm{N} \operatorname{cov}_{\mathrm{i}}=\mathrm{Nq}_{\mathrm{i}} \mathrm{q}_{\mathrm{j}} \mathrm{p}_{\mathrm{O} i \mathrm{i}} \mathrm{p}_{\mathrm{Ai}} \mathrm{p}_{\mathrm{oj}}\left(\mathrm{p}_{\mathrm{Aj \textrm {i }}}-\mathrm{p}_{\mathrm{Aj}}\right)
$$

It should be pointed out, that apart from $\mathrm{p}_{\mathrm{Aij}}$ and $\mathrm{p}_{\mathrm{A}}$ all probabilities appear only in pairs as products of $\mathrm{p}_{\mathrm{i}}=\mathrm{p}_{0 \mathrm{i}} \mathrm{p}_{\mathrm{Ai}}$ (see equ. M9, M10 and M11). Therefore the noise properties depend mainly on this product.

Based on the derived equations, the parameters of interest, which are $\mathrm{q}$ and $\mathrm{N}$, can be determined from mean, variance and covariance:

a) Combining mean (see eq. M9) and variance (see eq. M10) yields the classical parabolic variance versus mean relationship as originally introduced for nonstationary channel noise analysis (Sigworth 1980) in case that the quantal size $\mathrm{q}_{\mathrm{i}}$ is constant and does not depend on the stimulus number:

$$
\operatorname{Var}_{i}=q_{i} \bar{Y}_{i}-\frac{1}{N} \bar{Y}_{i}^{2}
$$

Fitting this to a parabolic plot, the initial slope yields the quantal size $\mathrm{q}$ and the width of the parabola gives the number of release sites $\mathrm{N}$. 
Dividing eq. M12 by the mean yields a linear relationship between variance over mean ratio versus mean (Heinemann and Conti 1992):

$$
\frac{\operatorname{Var}_{i}}{\bar{Y}_{i}}=q_{i}-\frac{1}{N} \bar{Y}_{i}
$$

This linearization of the classical parabolic variance versus mean relationship allows to apply fitting procedures, which consider $y$ - and $\mathrm{x}$-axis errors for weighted fitting (Orear 1982) in order to determine the parameters $\mathrm{q}_{\mathrm{i}}$ and $\mathrm{N}$ from the $\mathrm{y}$-axis intercept and the slope, respectively.

The presence of quantal size variation introduces additional factors to the terms in the variance versus mean relationship, as shown in appendix A. Accordingly the parameters q' and N' obtained from fits to the variance versus mean plot or to the ratio of variance over mean versus mean plots, replace the true quantal size $q$ and number of release sites $\mathrm{N}$ (eq. A24 and eq. A25):

$$
\begin{gathered}
\mathrm{q}_{\mathrm{i}}^{\prime}=<\mathrm{q}_{\mathrm{i}}>_{\text {Inter }}\left(1+\mathrm{CV}_{\text {Intra }}^{2}\right)\left(1+\mathrm{CV}_{\text {Inter }}^{2}\right) \\
\mathrm{N}^{\prime}=\mathrm{N}\left(1+\mathrm{CV}_{\text {Inter }}^{2}\right)^{-1}
\end{gathered}
$$

where $\langle\mathrm{q}\rangle_{\text {Inter }}$ is the mean quantal size, $\mathrm{CV}_{\text {Intra }}$ and $\mathrm{CV}_{\text {Inter }}$ are the coefficients of variation of intra- and intersite quantal variabiliy, respectively.

In the presentation of the results the EPSC amplitude variance versus mean plot will be referred to as variance-mean plot, and the plot of the ratio of the EPSC amplitude variance over mean versus mean plot as variance/mean-mean plot.

b) $\mathrm{N}$ can be calculated from the mean and covariance of consecutive EPSCs. In case of effectively no refilling, i.e. $b_{\mathrm{i} j \mathrm{j}}=0$, which is expected for two stimuli in very rapid succession, it follows from eq. M11

$$
\operatorname{Cov}_{\mathrm{i}, \mathrm{j}}=-\mathrm{Nq}_{\mathrm{i}} \mathrm{p}_{\mathrm{Oi}} \mathrm{p}_{\mathrm{Ai}} \mathrm{q}_{\mathrm{j}} \mathrm{p}_{\mathrm{Oj}} \mathrm{p}_{\mathrm{Aj}}
$$


Thus dividing the negative product of the mean amplitudes of consecutive EPSCs (see eq. M9) by the covariance between them (see eq. M16) yields the number of release sites

$$
\mathrm{N}=-\frac{\overline{\mathrm{Y}} \overline{\mathrm{Y}}_{\mathrm{i}+1}}{\operatorname{Cov}_{\mathrm{i}, \mathrm{i}+1}}
$$

In the presence of quantal size variation, the number N' obtained by this approach is related to the true number of release sites $\mathrm{N}$ by (see appendix A, eq. A27)

$$
\mathrm{N}^{\prime}=\mathrm{N}\left(1+\mathrm{CV}_{\text {Inter }}^{2}\right)^{-1}
$$

c) The quantal size q can be calculated for each response in the train of stimuli from the mean, variance and covariance of consecutive EPSCs. In the case of effectively no refilling an expression for the actual quantal size for each response in the train can be derived from the covariance (see eq. M16), mean and variance (see eqs. M9,M10). Division of eq. M16 for consecutive responses $i$ and $i+1$ by eq. M9 for response $i$ or i+1 yields

$$
\begin{aligned}
\frac{\operatorname{Cov}_{\mathrm{i}, \mathrm{i}+1}}{\overline{\mathrm{Y}}_{\mathrm{i}}}=-\mathrm{q}_{\mathrm{i}+1} \mathrm{p}_{\mathrm{Ai+1}} \mathrm{p}_{\mathrm{oi}+1} \\
\text { and } \frac{\operatorname{Cov}_{\mathrm{i}, \mathrm{i}+1}}{\overline{\mathrm{Y}}_{\mathrm{i}+1}}=-\mathrm{q}_{\mathrm{i}} \mathrm{p}_{\mathrm{oi}} \mathrm{p}_{\mathrm{Ai}}
\end{aligned}
$$

Substitution of eq. M9 into eq. M13 and writing it for response i and i+1 gives

$$
\begin{gathered}
\frac{\operatorname{Var}_{i}}{\bar{Y}_{i}}=q_{i}-q_{i} p_{o i} p_{A i} \\
\text { and } \quad \frac{\operatorname{Var}_{i+1}}{\bar{Y}_{i+1}}=q_{i+1}-q_{i+1} p_{\text {oit1 }} p_{\text {Ai }+1}
\end{gathered}
$$


Solving eq. M20a and M20b for $\mathrm{q}_{\mathrm{i}}$ and $\mathrm{q}_{\mathrm{i}+1}$, and substituting eq. M19b and M19a, respectively, yields

$$
\begin{aligned}
\mathrm{q}_{\mathrm{i}} & =\frac{\operatorname{Var}_{\mathrm{i}}}{\overline{\mathrm{Y}}_{\mathrm{i}}}-\frac{\operatorname{Cov}_{\mathrm{i}, \mathrm{i}+1}}{\overline{\mathrm{Y}}_{\mathrm{i}+1}} \\
\text { and } \quad \mathrm{q}_{\mathrm{i}+1} & =\frac{\operatorname{Var}_{\mathrm{i}+1}}{\overline{\mathrm{Y}}_{\mathrm{i}+1}}-\frac{\operatorname{Cov}_{\mathrm{i}, \mathrm{i}+1}}{\overline{\mathrm{Y}}_{\mathrm{i}}}
\end{aligned}
$$

Now setting $\mathrm{i}+1$ in eq. $21 \mathrm{~b}$ back to $\mathrm{i}$ yields two independent equations for $\mathrm{q}_{\mathrm{i}}$

$$
\mathrm{q}_{\mathrm{i}}=\frac{\operatorname{Var}_{\mathrm{i}}}{\overline{\mathrm{Y}}_{\mathrm{i}}}-\frac{\operatorname{Cov}_{\mathrm{i}-1, \mathrm{i}}}{\overline{\mathrm{Y}}_{\mathrm{i}-1}}
$$

Applying these equations to estimate the quantal size for each respose in the train eq. M21a was used for response 1, the mean of eq. M21a and M21c for responses 2 to 4 and eq. M21c for response 5.

Again, in the presence of quantal size variation, this approach yields the parameter q', which is related to the true quantal size average by (see appendix A, eq. A30)

$$
\mathrm{q}^{\prime}=<\mathrm{q}>_{\text {Inter }}\left(1+\mathrm{CV}_{\text {Intra }}^{2}\right)\left(1+\mathrm{CV}_{\text {Inter }}^{2}\right)
$$

In order do distinguish the values determined for q' and N' by applying the different approaches, in the documentation and discussion of the results $\mathrm{q}^{*}$ and $\mathrm{N}^{*}$ refer to values obtained applying the variance-mean method and $\mathrm{q}^{* *}$ and $\mathrm{N}^{* *}$ refer to values obtained with the approach including the covariance.

\subsection{Monte Carlo Simulation}

This simulation of synaptic transmission is based on the binomial model as described above. The simulation procedure is performed on an array of independent release sites and has three components: Release sites can be in different states, which are releasecompetent or not. The first component computes the transitions of release sites between the possible states (pool kinetics) in-between evoked release events and 
neglects any spontaneous release. The second component simulates evoked release. For sites in a release competent state, it computes whether or not a release event occurs based on a probability distribution generated beforehand. If a release event occurs the site is set to the empty state. The third component assigns a quantal size to each release event based on a quantal size distribution. The three components are presented separately in the following.

\subsubsection{Pool kinetics}

The pool size is the number of sites being in a certain state or having a certain property. Here two different models were considered. The first is a two-state model (see Fig. 4A), where every site is either in the empty or a release competent occupied state, and each state can have a certain assigned property regarding release probability and quantal size (see Fig. 4B). The second is a three-state model, where transitions occur between the empty and two occupied states with different assigned properties:

A

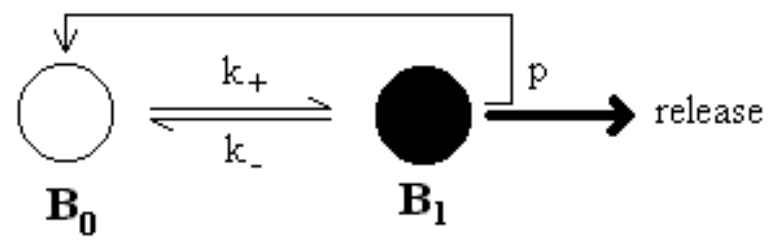

B

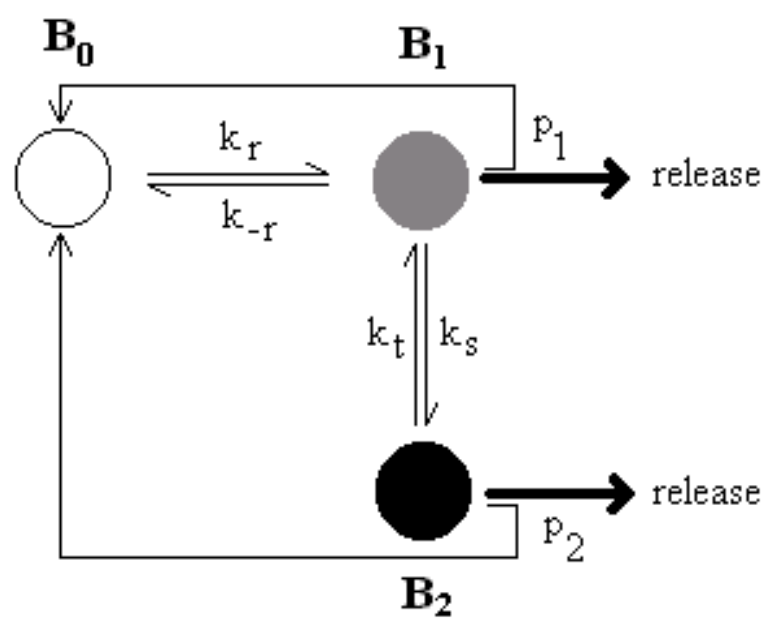

Fig. 4: Schemes describing the pool models used in the simulations. (A) Two-state pool model, where each release site is either in the occupied state or in the empty state. (B) Three-state pool model, where each release site is either in the empty state or one of two occupied states, of which the second state is reached via the first. 
a) Two-state model

Assume $\mathrm{N}$ release sites, to which vesicles dock or undock. Docking occurs to empty release sites with rate constant $k_{+}$and undocking occurs from occupied sites with rate constant k. This is summarised in the kinetic scheme in Fig. 4A. The kinetic, continuous time, differential equation for the number of release sites in the occupied state $\mathrm{B}_{1}$ and the number of release sites in the empty state $\mathrm{B}_{0}$ is

$$
\frac{\mathrm{dB}_{1}}{\mathrm{dt}}=\mathrm{k}_{+} \mathrm{B}_{0}-\mathrm{k}_{-} \mathrm{B}_{1}
$$

with the constraint $\quad \mathrm{N}=\mathrm{B}_{1}+\mathrm{B}_{0}$

where $B_{1}$ is the number of occupied sites and $B_{0}$ is the number of empty sites. This equation holds during the time intervals between two stimuli in the absence of spontaneous release events. The solution of the equations is

$$
B_{1}(t)=B_{1}(\infty)\left(1-e^{-\frac{t}{\tau}}\right)+B_{1}(0) e^{-\frac{t}{\tau}}
$$

$$
\begin{array}{ll}
\text { with steady state occupancy } & \mathrm{B}_{1}(\infty)=\frac{\mathrm{k}_{+}}{\mathrm{k}_{+}+\mathrm{k}_{-}} \mathrm{N} \\
\text { and time constant } & \tau=\frac{1}{\mathrm{k}_{+}+\mathrm{k}_{-}}
\end{array}
$$

Expressing the rate constants in terms of steady state occupancy $B_{1}(\infty)$ and the recovery time constant $\tau$ gives

$$
\begin{aligned}
& \mathrm{k}_{+}=\frac{\mathrm{B}_{1}(\infty)}{\mathrm{N}} \tau \\
& \mathrm{k}_{-}=\frac{1}{\tau}-\mathrm{k}_{+}
\end{aligned}
$$


The approximate probabilities for the transition of a release site from the empty to the occupied state and vice versa during a very small time step $\Delta \mathrm{t}$ are:

$$
\begin{aligned}
& \mathrm{p}_{\text {in }}=\mathrm{k}_{+} \Delta \mathrm{t} \\
& \mathrm{p}_{\text {out }}=\mathrm{k}_{-} \Delta \mathrm{t}
\end{aligned}
$$

For the simulation discrete time has to be introduced. The time step size must be chosen such that the probability for a combined site transition, i.e. from empty via occupied back to empty or vice versa, is low:

$$
\begin{gathered}
\mathrm{p}_{\text {in }} \cdot \mathrm{p}_{\text {out }}<<1 \\
\text { or } \quad \mathrm{p}_{\text {in }} \cdot \mathrm{p}_{\text {out }}=\text { limit }
\end{gathered}
$$

Substitution of eq. 8 and eq. 9 gives

$$
\text { limit }=\mathrm{k}_{+} \mathrm{k}_{-} \Delta \mathrm{t}^{2}
$$

Finally solving eq. 12 for the time step size yields

$$
\Delta \mathrm{t}=\sqrt{\frac{\text { limit }}{\mathrm{k}_{+} \mathrm{k}_{-}}}
$$

b) Three-state model

This is taken from the most recent model for synaptic transmission in the calyx of Held synapse presented by Trommershäuser (2000). It was applied as the most realistic simulation scenario available. The documentation of the three-state model is equivalent to the one given in (Trommershäuser, 2000). Here release sites undergo transitions between the empty and two occupied states (see Fig. 4B). The kinetic, continuous time differential equations for the number of sites in the occupied state 
one, $\mathrm{B}_{1}$, the number in occupied state two, $\mathrm{B}_{2}$, and the number in the empty state $\mathrm{B}_{0}$ in-between stimuli are as follows

$$
\begin{aligned}
& \frac{\mathrm{dB}_{1}}{\mathrm{dt}}=-\left(\mathrm{k}_{\mathrm{s}}+\mathrm{k}_{-\mathrm{r}}\right) \mathrm{B}_{1}+\mathrm{k}_{\mathrm{r}} \mathrm{B}_{0}+\mathrm{k}_{\mathrm{t}} \mathrm{B}_{2} \\
& \frac{\mathrm{dB}}{\mathrm{dt}}=-\mathrm{k}_{\mathrm{t}} \mathrm{B}_{2}+\mathrm{k}_{\mathrm{s}} \mathrm{B}_{1}
\end{aligned}
$$

$\mathrm{B}_{0}$ and the rate constants are defined by the two time constants of recovery, which are observed in experiments, and the fraction of occupied sites

$$
\mathrm{F} \equiv \frac{\mathrm{B}_{1}+\mathrm{B}_{2}}{\mathrm{~B}_{0}+\mathrm{B}_{1}+\mathrm{B}_{2}}
$$

and the steady-state ratio between the size of the two different occupied pools using a parameter $\mathrm{R}$ with $0<\mathrm{R}<1$, such that

$$
\frac{B_{1}}{B_{2}}=\frac{R}{1-R}
$$

The solution of the kinetic equations is given in detail in (Trommershäuser 2000). The rate constants expressed in terms of the time constants and constraints are

$$
\begin{gathered}
\mathrm{k}_{-\mathrm{r}}=\frac{1-\mathrm{F}}{2(1-\mathrm{F}+\mathrm{FR})}\left[\frac{1}{\tau_{1}}+\frac{1}{\tau_{2}}+\sqrt{\left(\frac{1}{\tau_{1}}+\frac{1}{\tau_{2}}\right)^{2}+\frac{4}{\mathrm{R} \tau_{1} \tau_{2}}(1-\mathrm{F}+\mathrm{FR})}\right] \\
\mathrm{k}_{\mathrm{s}}=(\mathrm{R}-1)\left[\mathrm{k}_{-\mathrm{r}} \frac{1+\mathrm{F}(\mathrm{R}-1)}{1-\mathrm{F}}-\frac{1}{\tau_{1}}-\frac{1}{\tau_{2}}\right] \\
\mathrm{k}_{\mathrm{r}}=\mathrm{k}_{-\mathrm{r}} \frac{\mathrm{FR}}{1-\mathrm{F}}
\end{gathered}
$$




$$
\mathrm{k}_{\mathrm{t}}=\mathrm{k}_{\mathrm{s}} \frac{\mathrm{R}}{1-\mathrm{R}}
$$

The transition with the fastest rate constants determines the size of the discrete time step, see above. For the given data this was

$$
\Delta \mathrm{t}=\sqrt{\frac{\operatorname{limit}}{\mathrm{k}_{\mathrm{r}} \mathrm{k}_{-\mathrm{r}}}}
$$

The approximate transition probabilities during a small discrete simulation time step are then

$$
\begin{aligned}
& \mathrm{p}_{-\mathrm{r}}=\mathrm{k}_{-\mathrm{r}} \Delta \mathrm{t} \\
& \mathrm{p}_{\mathrm{s}}=\mathrm{k}_{\mathrm{s}} \Delta \mathrm{t} \\
& \mathrm{p}_{\mathrm{r}}=\mathrm{k}_{\mathrm{r}} \Delta \mathrm{t} \\
& \mathrm{p}_{\mathrm{t}}=\mathrm{k}_{\mathrm{t}} \Delta \mathrm{t}
\end{aligned}
$$

The simulation procedure was then as follows: The number of pool kinetic simulation steps between the computations of release events was calculated on basis of eq. S13, or eq. S19, depending on the desired inter-stimulus time and the recovery time between trains of stimuli. After each evoked release computation, the pool dynamics simulation was run the assigned number of steps. For each site in each step a number between 0 and 1 was drawn with a random number generator (Igor Pro, Wave Metrics) and compared to the calculated transition probabilities $\mathrm{p}_{\text {in }}$ or $\mathrm{p}_{\text {out }}$ according to the state of the actual release site. If the drawn number was smaller than the probability to be applied a transition of the state of the release site occurred. 


\subsubsection{Evoked release simulation and release probability distributions}

Release upon a stimulus was computed on sites in the release competent state. For such sites a random number between 0 and 1 was generated. If that number was lower than the release probability $\mathrm{p}$ assigned to that site or the state of that site, the site was set to the empty state and a quantal unit was added to a parameter for integration of the overall evoked release outcome. In the simplest case the release probability was set to the same value for all release sites and for all stimuli in the train. Heterogeneous release probability was introduced by assigning different probabilities to different sites or to different release site states. An increase in the release probability with successive stimuli, i.e. facilitation, was introduced by assigning different probabilities to the respective evoked release computations in the stimulation. As a realistic scenario for heterogeneity and facilitation the results by Trommershäuser (2000) were taken again: Residual bulk $\mathrm{Ca}^{2+}$ build-up during stimulation is at stimulus $\mathrm{i}$ (Helmchen et al. 1997)

$$
\begin{gathered}
\Delta_{\text {res }} \mathrm{Ca}^{2+}(1)=0 \\
\text { and } \quad \Delta_{\text {res }} \mathrm{Ca}^{2+}(\mathrm{i})=\mathrm{x}_{0} \sum_{\mathrm{n}=1}^{\mathrm{i}} \exp \left(-\frac{\Delta \mathrm{t}(\mathrm{n}-1)}{\tau_{\mathrm{x}}}\right) ; \quad \text { for } \mathrm{i}>1
\end{gathered}
$$

The $\left[\mathrm{Ca}^{2+}\right]$ at the location of a release site is given by an empirical equation according to (Trommershäuser 2000)

$$
\left[\mathrm{Ca}^{2+}\right]_{\mathrm{RS}, \mathrm{j}}=\mathbf{J}_{\mathrm{Ca}}^{\max } \frac{\left[\mathrm{Ca}^{2+}\right]_{\text {out }}}{\left[\mathrm{Ca}^{2+}\right]_{\text {out }}+\mathrm{EC}_{50}}\left[\delta_{\mathrm{j} 2}+\eta\left(1-\frac{\gamma}{1+\frac{\Delta_{\mathrm{res}} \mathrm{Ca}^{2+}}{\mathrm{K}_{\mathrm{D}}}}\right)\right]
$$

The term outside the brackets is a Michaelis-Menten-type relationship between extracellular calcium concentration $\left[\mathrm{Ca}^{2+}\right]_{\text {ext }}$ and calcium influx upon stimulation (Schneggenburger et al. 1999) and allows to simulate situations with different $\left[\mathrm{Ca}^{2+}\right]_{\text {ext. }}$ The Kronecker delta in the brackets distinguishes the case of a release site being co-localised with the source of $\mathrm{Ca}^{2+}$-influx, i.e. a $\mathrm{Ca}^{2+}$-channel, $\left(\delta_{22}=1\right)$, and 


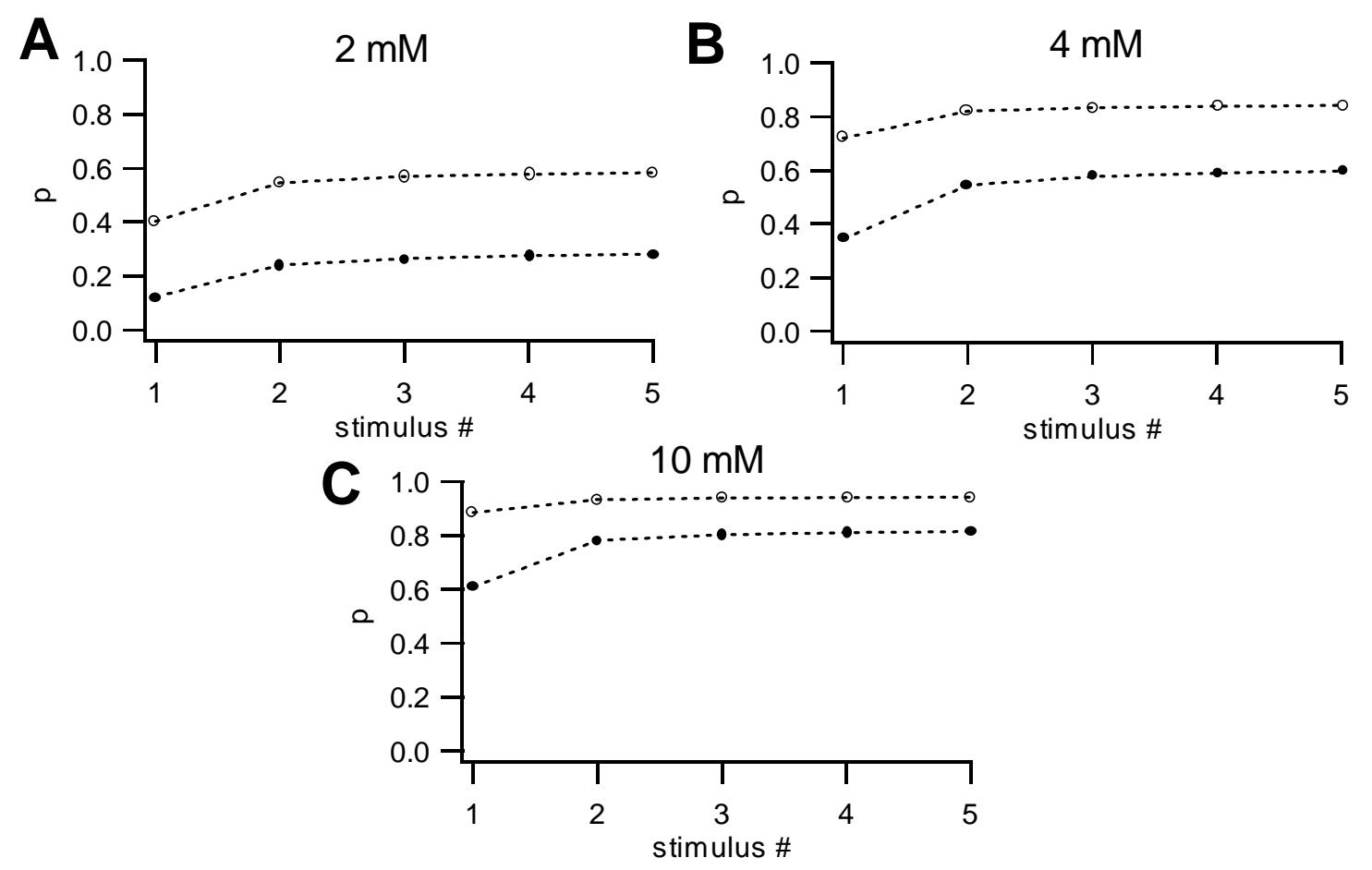

Fig. 5: Modelling of facilitation. Release probabilities as calculated from eq. S21, S22 and S23 for a train of five stimuli at $100 \mathrm{~Hz}$. Two cases were distinguished: Colocalization of the release site with a $\mathrm{Ca}^{2+}$-channel (filled circles) and no colocalization (open circles). (A) Situation in $2 \mathrm{mM}\left[\mathrm{Ca}^{2+}\right]_{\mathrm{ext}}$. (B) Situation in $4 \mathrm{mM}$ $\left[\mathrm{Ca}^{2+}\right]_{\mathrm{ext}}$. (C) Situation at $10 \mathrm{mM}\left[\mathrm{Ca}^{2+}\right]_{\mathrm{ext}}$.

the case of a release site sensing only ambient $\left[\mathrm{Ca}^{2+}\right]\left(\delta_{12}=0\right)$. This is one possibility to introduce heterogeneity in $\mathrm{p}$. The second term in the brackets accounts for the accumulation of ambient $\left[\mathrm{Ca}^{2+}\right]$ (see eq. S21), which causes a facilitation of the release probability (see eq. S23) with repetitive stimulation.

The actual release probability is then according to a classical Hill equation

$$
\mathrm{p}_{\mathrm{i}, \mathrm{j}}=\frac{\left[\mathrm{Ca}^{2+}\right]_{\mathrm{RS}}^{4}}{\left[\mathrm{Ca}^{2+}\right]_{\mathrm{RS}}^{4}+\mathrm{K}_{1 / 2}^{4}}
$$

Values for $\mathrm{J}_{\mathrm{Ca} \text {,max }}$ and $\mathrm{EC}_{50}$ have been measured by Schneggenburger et al. (1999), while $K_{1 / 2}, \eta, \gamma$ and $K_{D}$ are free parameters of the model. The applied parameters are summarised in appendix B. For more details refer to (Trommershäuser 2000). In Fig. 5 the release probabilities in a five stimulus train at $100 \mathrm{~Hz}$ in 2,4 and $10 \mathrm{mM}$ $\left[\mathrm{Ca}^{2+}\right]_{\mathrm{ext}}$ are displayed (open symbols: co-localised sites, i.e. $\delta_{22}=1$; closed symbols: sites sensing only ambient $\left[\mathrm{Ca}^{2+}\right]$, i.e. $\delta_{12}=0$ ), which were applied in some of the simulations. 


\subsubsection{Quantal size assignment}

Quantal variation was taken into account based on mEPSC amplitude data kindly provided by A. Meyer (see Fig. 6). Both intra- and intersite quantal variability were considered. The mEPSC amplitude values were sorted according to increasing size and normalised such that the mean amplitude was equal to one. The ranking numbers of the sorted data were used for assigning a quantal size to release events. The simulations required ranking number sets of a certain size, which still represent or sample the complete mEPSC amplitude range. For this the sorted amplitude data set versus original ranking number was extended or compressed by interpolation, such that an amplitude data set with the desired number of ranking numbers was obtained, as shown in Fig. 3C.

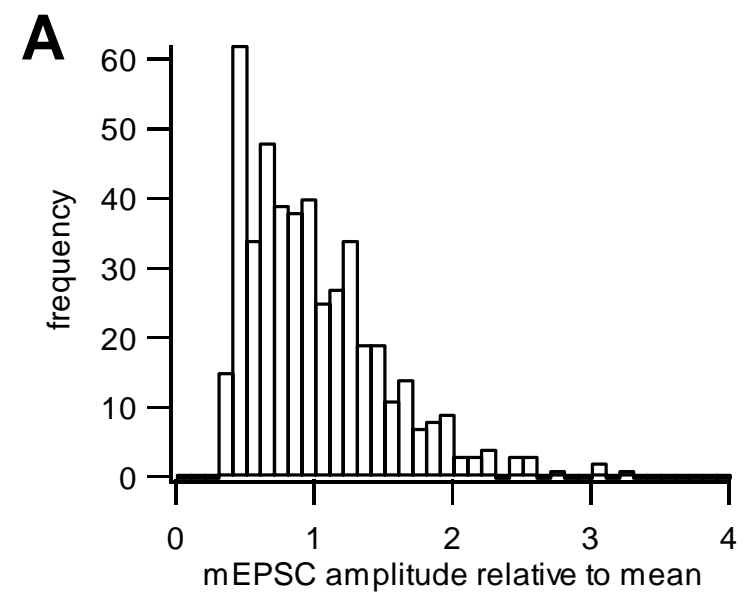

B

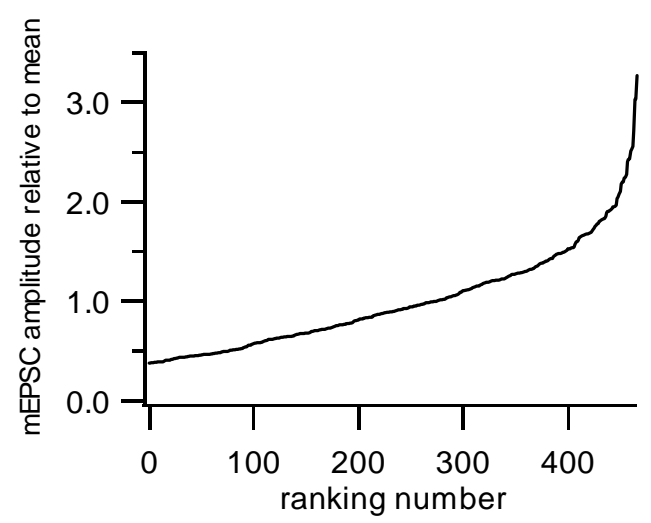

\section{C}

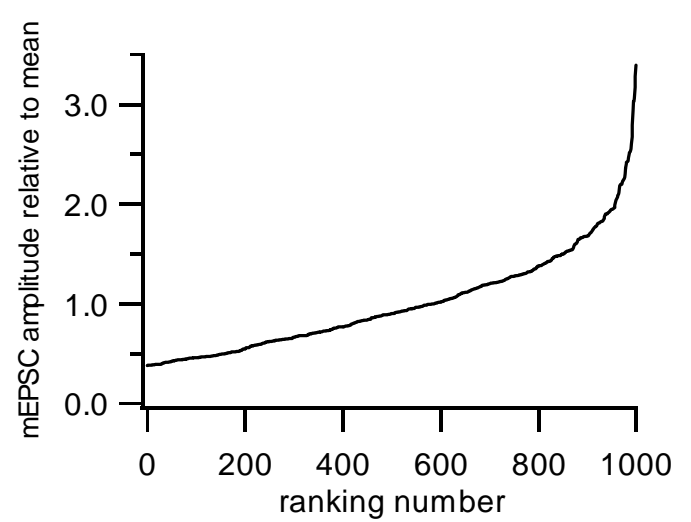

Fig. 6: The quantal size data used in the simulations. (A) mEPSC histogram normalized to give a mean of one. (B) Original mEPSC data set of 468 amplitude values, sorted and ranked according to increasing amplitude (C) mEPSC amplitude data set extended to 1000 ranking numbers for the simulations. 
For intrasite quantal variability the sorted mEPSC amplitude data was extended to 1000 ranking numbers. If a release event had been established, its amplitude was determined by generating a random number between 0 and 999 and choosing the amplitude sample assigned to that ranking number.

For the case of intersite quantal variability the sorted mEPSC amplitude data was extended to as many ranking numbers as release sites were present. Each release site was assigned a single quantal size sample. In some simulations heterogeneity in the release probability was introduced, by dividing the release sites into two groups of equal size, assigning each group a different release probability. In the case of intersite quantal variability, there could be a problem regarding the introduction of bias in sampling the quantal size distribution, if there would be a correlation between quantal sitze and release probability. In order to maintain the independence of quantal size and release probability, the sorted mEPSC data was extended for each group separately for as many ranking numbers as release sites were present in the respective group. This ensures that each group of release sites samples the complete range of quantal sizes. The simulation routines for analysis were programmed and executed with the program IgorPro (Wavemetrix, Oregon, USA).

\subsection{Electrophysiological Recordings}

To measure synaptic transmission at the calyx of Held synapse electrophysiological recordings were performed in rat brain stem slices. APs were elicited in the presynaptic terminal applying voltage pulses to the afferent fibers and the evoked postsynaptic current was measured applying the patch-clamp technique in the wholecell mode (Marty and Neher 1995). In this configuration ion currents across the entire cell are recorded. Synaptic transmission was quantified by the ionic currents, which arise from the opening of voltage gated ion channels upon binding of neurotransmitter. 


\subsubsection{Solutions}

If not otherwise stated chemicals and drugs were purchased from Sigma, Germany.

a) standard external solution (in $\mathrm{mM}$ ):

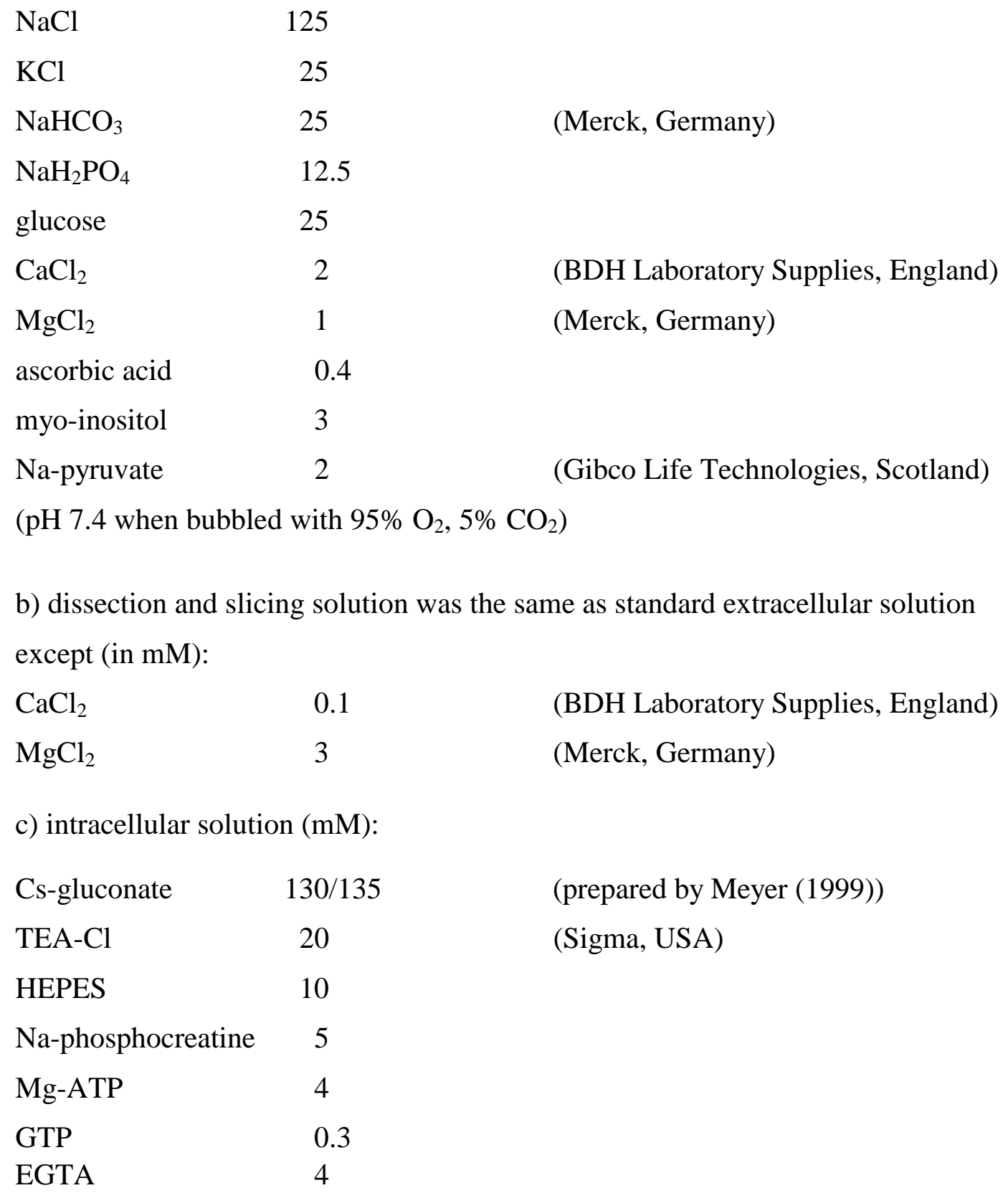

(pH 7.2, adjusted with CsOH 1M, 295-300 mosm)

drugs were added to the standard extracellular solution at the following concentrations:

$\begin{array}{lrr}\text { DAP-5 } & 50 \mu \mathrm{M} & \text { (Tocris, USA) } \\ \text { CTZ } & 100 \mu \mathrm{M} & \text { (Tocris, USA) } \\ \text { Kyn } & 1 \mathrm{mM} & \text { (Sigma, USA) }\end{array}$




\subsubsection{Dissection and slice preparation}

The preparation of transverse brainstem slices followed the procedure described in (Edwards et al. 1989) 8- to 10-day-old Wistar rats were decapitated. Dissection and slicing procedures were performed in low Ca dissection solution, which was cooled to freezing point. The whole brain was removed from the skull. The brainstem was separated from the cortex by a slightly inclined transverse cut (see Fig. 7A). The meninges and blood vessels were removed from the ventral surface of the brainstem. The brainstem, including the cerebellum, was glued by the anterior cut surface onto the Teflon block in the slicing chamber with super glue (Art.-Nr. 461305, UHU $\mathrm{GmbH}$, Baden, Germany). Before filling the slicing chamber with solution the Teflon block was positioned such that the fibres projecting from the aVCN onto the MNTB, which appeared as a white band on the ventral side of the brain stem, ran parallel to

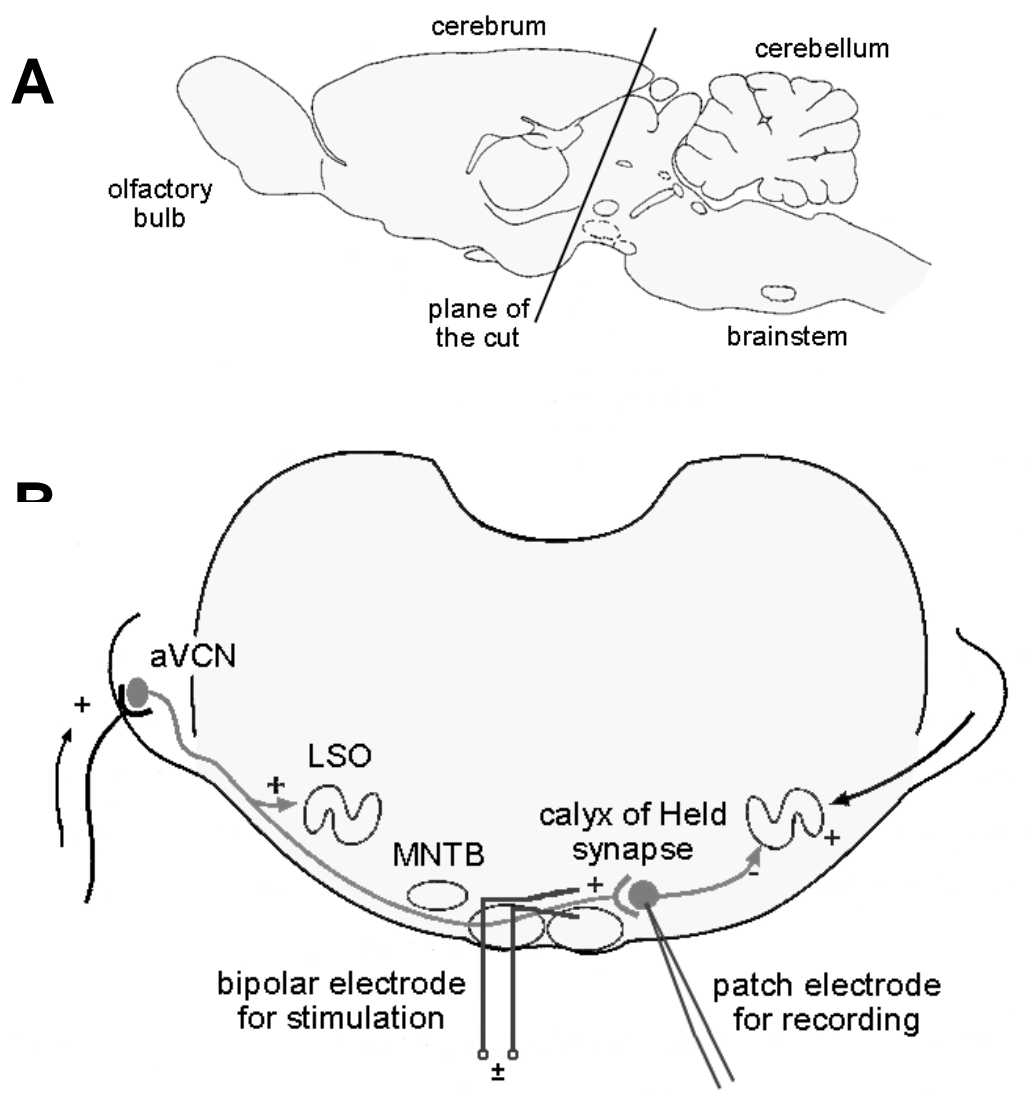

Fig. 7: Documentation of the dissection procedure and the recording configuration. (A) Position of the inclined transverse cut separating the brainstem from the cortex. (B) View on the plane of a brainstem slice with the positions of stimulation and recording electrodes. Axon collaterals from globular cells of the aVCN (anterior ventral cochlear nucleus) terminate on the principal neurons of the MNTB (medial nucleus of the trapezoid body) in the calyx of Held synapse. (LSO: lateral superior olive). 
the slicing plane. Transverse slices were cut with a vibratome (Campden Instruments, Great Britain) proceeding in a ventral to dorsal direction. In this configuration, the $7^{\text {th }}$ nerve tract (Nervus facialis) marked the start of the superior olivary complex, with the

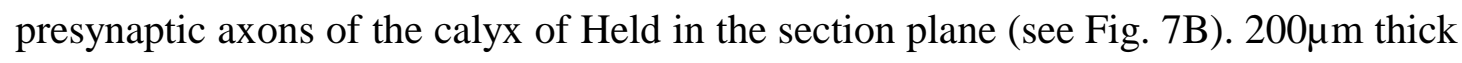
slices containing the MNTB were cut over a total extent of up to $1 \mathrm{~mm}$. Immediately after cutting, each slice was transferred to an incubation chamber containing standard external solution continuously bubbled with carbogen and maintained at $37^{\circ} \mathrm{C}$ for four hours.

\subsubsection{Electrophysiology}

Slices were placed into the recording chamber and covered with a platinum grid to prevent any dislocation. The recording chamber was perfused with solutions from a gravity-fed system of syringes and Teflon tubing at different speeds allowing solution exchange or continuous perfusion. The solution level in the chamber was controlled with an overflow tubing by gravity-driven suction. Slices were visualised with an upright microscope (Axioskop, Zeiss, Oberkochem, Germany) with a 5x objective $(\mathrm{NA}=0.15$, Plan-Neofluar, Zeiss, Oberkochem, Germany) for pre-assembly and with a 60X water immersion objective (NA = 0.9, LUMPlanFl, Olympus, Japan) applying the IR-DIC mode for recordings. For afferent fibre stimulation bipolar stimulation electrodes were either purchased (Concentric bipolar stimulation electrodes; outer contact diameter, $150 \mu \mathrm{m}$; inner contact diameter, $25 \mu \mathrm{m}$; length: $50 \mathrm{~mm}$; Rhodes Medical Instruments, USA) or made from Teflon-coated platinum-iridium wires (Science Products, Hofheim, Germany) glued into a $\Theta$-capillary. The purchased concentric electrodes were slightly inserted into the afferent fibre bundle, while the self-made electrodes were positioned on the slice such that the fibre bundle was located in between the tips of the two wires. Stimulation pulses were generated with an isolated pulse stimulator (A-M Systems, Model 2100, Kirkland, Washington, USA). Voltage pulses were $200 \mu \mathrm{s}$ long with amplitudes between 4 and $30 \mathrm{~V}$. For recordings, the MNTB cells had to be identified, of which the input was activated by the fibre stimulation. Cells were screened using a patch pipette filled with standard extracellular solution for extracellular recording of action potentials. Only those cells were chosen for whole-cell recordings, which showed extracellularly recorded preand postsynaptic action potentials elicited by afferent fibre stimulation. 
Patch pipettes were pulled from borosilicate glass (diameter $2 \mathrm{~mm}$, wall thickness 0.3 mm, Hilgenberg, Malsfeld, Germany) and coated with Sylgard. Heat polishing was applied in some cases, but there was no significant advantage observed for Giga-seal formation or stability of recordings. The open tip resistance was 2 to $4 \mathrm{M} \Omega$. Cells were voltage-clamped at $-80 \mathrm{mV}$ holding potential and EPSCs were measured with a EPC-9 patch clamp amplifier (HEKA, Lambrecht, Pfalz, Germany). 50 to $85 \%$ online access resistance compensation was applied, such that the uncompensated access resistance never exceeded $3 \mathrm{M} \Omega$. Access resistance tended to increase during recordings and the $\mathrm{R}_{\mathrm{s}}$ compensation was readjusted either manually or automatically with the auto $\mathrm{C}$-slow mode. The range of $R_{\mathrm{s}}$ accepted for analysis was 4 to $12 \mathrm{M} \Omega$ before compensation (in exceptions $20 \mathrm{M} \Omega$ ). The stimulation protocols were single stimuli at $0.25 \mathrm{~Hz}$ for stationary statistical data, trains of five stimuli at $100 \mathrm{~Hz}$ repeated at $0.1 \mathrm{~Hz}$ for non-stationary statistical data, and trains of 20 stimuli at $100 \mathrm{~Hz}$ repeated at $0.025 \mathrm{~Hz}$ for pool estimation. Currents were sampled at $50 \mathrm{kHz}$ and filtered at $6 \mathrm{kHz}$.

\subsection{Analysis}

Routines for analysis were written and executed with the program IgorPro (Wavemetrics, Oregon, USA). As first step of the analysis, raw data traces were compensated offline for clamp errors due to access resistance and membrane capacity (Traynelis, 1998), assuming a reversal potential of $+10 \mathrm{mV}$ for the EPSCs. Release can be quantified as peak EPSC amplitude or charge of the EPSC current transient (Walmsley 1993). Here the EPSC peak amplitude was used (see discussion in results chapter). The EPSC peak current location was determined after the corresponding stimulus within a defined interval. For this a peak detector routine based on the first derivative and second derivative of the current trace was applied. Before peak detection traces were four times boxcar smoothed with a window size equivalent to $100-410 \mu \mathrm{s}$. In contrast traces were only smoothed once for the actual current readings. The EPSC amplitude was calculated as the difference between peak and baseline current. The baseline current was determined as the average current over an interval of $0.2-0.5 \mathrm{~ms}$ directly preceding the EPSC in case of recordings under control conditions. In case of CTZ application, which prolongs the EPSC decay (see Results) such that they overlap in trains at $100 \mathrm{~Hz}$, double exponentials were fitted to the decay phase of the preceding EPSC, and the baseline current was taken from the 
extrapolation to the time of the actual EPSC peak. The stability of the obtained data was assessed by fitting a line to the amplitude of the first stimulus in the train plotted versus repetition number. For analysis only data sets were chosen which displayed less than $20 \%$ change in the regression line (see Oleskevich et al. 2000). To minimise contamination of the variance and covariance estimates by long term trends or drifts in the recording, the EPSC amplitude data sets were analysed for mean, variance and covariance by calculating these parameters over short segments or groups of sequential records and averaging the obtained values to give the overall or grand value of the parameter (see Clamann et al. 1989, Quastel 1997). There were several possibilities for dividing the data into segments, e.g. non-overlapping, independent segments, half-overlapping segments and maximally overlapping windows. The accuracy of the variance estimates using these three approaches was calculated depending on segment and data set size in order to determine an optimal segment size (see appendix C) and is here expressed as signal to noise ratio (SNR):

$$
\operatorname{SNR}\left(v_{\text {tot }}\right)=\frac{\left\langle v_{\text {tot }}>\right.}{\sqrt{\operatorname{Var}\left(v_{\text {tot }}\right)}}=\frac{1}{\sqrt{\text { factor }_{\mathrm{x}}(\mathrm{N}, \mathrm{R})}}
$$

The complete expression for parameter $\operatorname{factor}_{\mathrm{x}}(\mathrm{N}, \mathrm{R})$ is complex and can be found for each approach $\mathrm{x}$ in appendix A. For $\mathrm{N}=100$ and segment sizes between 2 and 10 the $\mathrm{SNR}\left(\mathrm{v}_{\text {tot }}\right)$ is shown in Fig. 8. Small segments can be expected to remove any contamination by long-term trends more effectively than large segments. But there is a trade-off between small segment size and signal to noise ratio in any of the three approaches apparent. However we decided to use maximally overlapping segments of size two (Clamann et al. 1989 used independent segments of size 5 and 6), which yields the maximal possible degree of exclusion of contamination by long term trends and drifts, but only relatively little higher SNR compared to larger segments. An estimate for the accuracy of covariance estimates is calculated in appendix D for the condition of maximally overlapping segments of size 2 . 


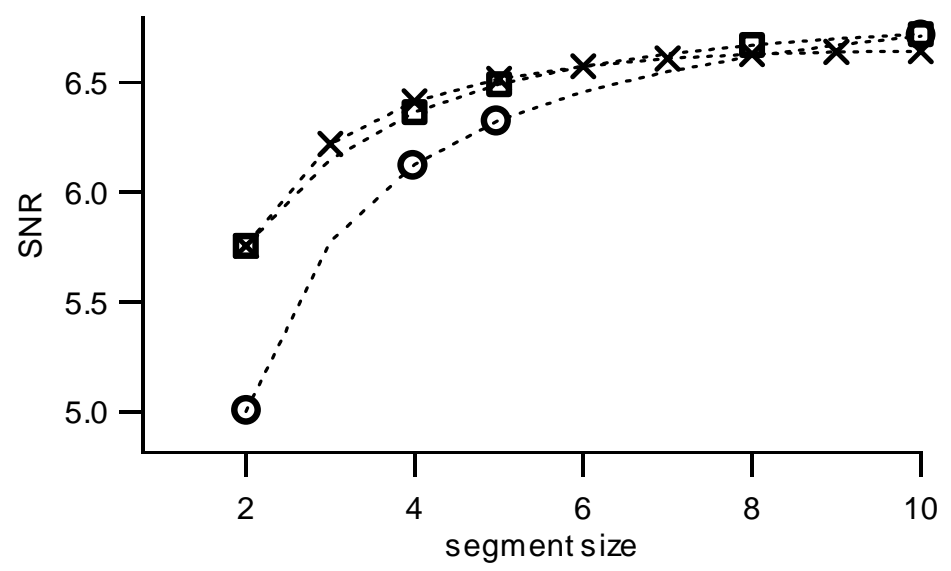

Fig. 8: SNR (signal to noise ratio) for the variance estimation based on the approach described in the analysis paragraph for a data set containing 100 samples: Independent, non-overlapping segments (circles); half-overlapping segments (squares); and completely overlapping segments (crosses).

For linear fits to the variance/mean-mean plots the procedure suggested by (Orear 1982) was used, which considers $y$ - and $x$ - axis data errors. Other fits were performed with the build-in procedure of Igor Pro. Fits to variance-mean plots were always constrained to pass the origin, because the observed baseline variance was two to four orders of magnitude smaller than the variance data of EPSCs and was therefore neglected (Clements and Silver 2000).

mEPSC analysis was performed with the event detection routine based on templatematching provided by the AxoGraph 4 software (Axon Instruments, Inc., Foster City, USA). If not otherwise stated results are reported as mean \pm SEM. 


\section{Results}

\subsection{Simulations}

Simulations of synaptic transmission were performed as described in the methods chapter in order to test the analytical approach and its performance in general and under conditions where basic assumptions are violated. The results of the simulations are summarised in Table 1 and Table 2. The simulations, which applied the two-state pool model, (see Table 1 and Table 2A), were performed with a recovery time constant of $4 \mathrm{~s}$, a train of 5 stimuli with $10 \mathrm{~ms}$ inter-stimulus interval, and an intertrain interval of $10 \mathrm{~s}$. The first set of conditions compared increasing homogenous release probabilities, $0.25,0.5$ and 0.75 , in the absence of quantal variability, in the presence of intrasite quantal variability and in the presence of intersite quantal variability for 500 release sites (see Table 1A). The second set of conditions tested was with homogenous release probability of 0.5 and increasing numbers of release sites, 250, 500, 750 and 1000, again for the three situations regarding quanta (see Table 1B). The third set of conditions compared was with 500 release sites and increasing heterogeneity in the release probability (see Table 1C). Half the sites were assigned a low and the other half of sites a high release probability, such that the average release probability was in all cases 0.5 . Here only intrasite and intersite quantal variability were tested. In Table $2 \mathrm{~A}$, results for simulations are summarised with $\mathrm{N}=500$ sites, in the absence of quantal variability, and in the presence of intraor intersite quantal variability, and applying the release probabilities at 2, 4, and 10 $\mathrm{mM}\left[\mathrm{Ca}^{2+}\right]_{\text {ext }}$ obtained from the facilitation model (see eq. S22 and S23; and Fig. 5). Half the sites were assigned the low and the other half the higher release probability. In Table 2B, the results of the three-state pool model are shown, where the probabilities of the facilitation model for $2 \mathrm{mM}$ and $4 \mathrm{mM}\left[\mathrm{Ca}^{2+}\right]_{\text {ext }}$ (see Fig. 5) were applied, and one release site state was assigned the lower probability and the other the higher one. Inter- and intrasite quantal variability were considered. The parameters applied for these simulations were taken from Trommershäuser (2000) as summarised in appendix B.

The determination of the quantal size $\mathrm{q}^{*}$ from parabolic fits to the variance-mean plots (see eq. M12) and from linear fits to variance/mean-mean plots (see eq. M13) appear to be extremely robust against any complication of the situation. The determination of 


\begin{tabular}{|c|c|c|c|c|c|c|c|c|c|}
\hline $\mathrm{N}$ & q' & $\mathrm{p}$ & \multicolumn{2}{|c|}{ var vs mean } & \multicolumn{2}{|c|}{ ratio vs mean } & \multicolumn{3}{c|}{ cov } \\
& & & $\mathrm{q}^{*}$ & $\mathrm{~N}^{*}$ & $\mathrm{q}^{*}$ & $\mathrm{~N}^{*}$ & $\mathrm{q}_{1}{ }^{* *}$ & $\mathrm{~N}_{1}{ }^{* *}$ & $\mathrm{~N}_{2}{ }^{* *}$ \\
\hline 500 & 1 & 0.25 & 1.03 & 446 & $1.03 \pm 0.09$ & $439 \pm 315$ & 1.01 & 486 & 540 \\
\hline 500 & 1 & 0.50 & 1.01 & 488 & $1.01 \pm 0.04$ & $490 \pm 158$ & 0.97 & 537 & 561 \\
\hline 500 & 1 & 0.75 & 1.00 & 506 & $1.01 \pm 0.03$ & $491 \pm 102$ & 0.95 & 562 & 882 \\
\hline 500 & 1.25 & 0.25 & 1.26 & 524 & $1.27 \pm 0.13$ & $506 \pm 581$ & 1.27 & 519 & 633 \\
\hline 500 & 1.25 & 0.50 & 1.26 & 541 & $1.26 \pm 0.06$ & $517 \pm 260$ & 1.30 & 491 & 618 \\
\hline 500 & 1.25 & 0.75 & 1.24 & 508 & $1.24 \pm 0.04$ & $520 \pm 174$ & 1.19 & 562 & 746 \\
\hline 500 & 1.25 & 0.25 & 1.24 & 432 & $1.25 \pm 0.12$ & $411 \pm 365$ & 1.28 & 388 & 431 \\
\hline 500 & 1.25 & 0.50 & 1.26 & 390 & $1.27 \pm 0.06$ & $383 \pm 135$ & 1.24 & 414 & 453 \\
\hline 500 & 1.25 & 0.75 & 1.25 & 399 & $1.25 \pm 0.04$ & $404 \pm 96$ & 1.21 & 428 & 666 \\
\hline
\end{tabular}

B

\begin{tabular}{|c|c|c|c|c|c|c|c|c|c|}
\hline $\mathrm{N}$ & q' & $\mathrm{p}$ & \multicolumn{2}{|c|}{ var vs mean } & \multicolumn{2}{|c|}{ ratio vs mean } & \multicolumn{3}{c|}{ cov } \\
& & & $\mathrm{q}^{*}$ & $\mathrm{~N}^{*}$ & $\mathrm{q}^{*}$ & $\mathrm{~N}^{*}$ & $\mathrm{q}_{1}{ }^{* *}$ & $\mathrm{~N}_{1}{ }^{* *}$ & $\mathrm{~N}_{2}{ }^{* *}$ \\
\hline 250 & 1 & 0.5 & 1.00 & 255 & $1.00 \pm 0.03$ & $252 \pm 60$ & 0.98 & 261 & 280 \\
\hline 500 & 1 & 0.5 & 1.01 & 488 & $1.01 \pm 0.04$ & $490 \pm 158$ & 0.97 & 537 & 561 \\
\hline 750 & 1 & 0.5 & 0.99 & 781 & $1.00 \pm 0.05$ & $745 \pm 294$ & 0.97 & 797 & 853 \\
\hline 1000 & 1 & 0.5 & 0.99 & 997 & $0.98 \pm 0.06$ & $1029 \pm 472$ & 0.97 & 1080 & 1033 \\
\hline 250 & 1.25 & 0.5 & 1.26 & 245 & $1.26 \pm 0.04$ & $247 \pm 83$ & 1.22 & 277 & 272 \\
\hline 500 & 1.25 & 0.5 & 1.26 & 541 & $1.26 \pm 0.06$ & $517 \pm 260$ & 1.30 & 491 & 618 \\
\hline 750 & 1.25 & 0.5 & 1.27 & 720 & $1.26 \pm 0.07$ & $766 \pm 457$ & 1.21 & 807 & 790 \\
\hline 1000 & 1.25 & 0.5 & 1.25 & 993 & $1.25 \pm 0.08$ & $1016 \pm 683$ & 1.20 & 1131 & 1010 \\
\hline 250 & 1.25 & 0.5 & 1.27 & 202 & $1.27 \pm 0.04$ & $198 \pm 52$ & 1.26 & 207 & 244 \\
\hline 500 & 1.25 & 0.5 & 1.26 & 390 & $1.27 \pm 0.06$ & $383 \pm 135$ & 1.24 & 414 & 453 \\
\hline 750 & 1.25 & 0.5 & 1.24 & 617 & $1.26 \pm 0.07$ & $573 \pm 241$ & 1.22 & 653 & 655 \\
\hline 1000 & 1.25 & 0.5 & 1.30 & 721 & $1.31 \pm 0.09$ & $695 \pm 322$ & 1.24 & 831 & 909 \\
\hline
\end{tabular}

C

\begin{tabular}{|c|c|c|c|c|c|c|c|c|c|c|}
\hline \multirow[t]{2}{*}{$\mathrm{N}$} & \multirow[t]{2}{*}{ q' } & \multirow[t]{2}{*}{$\langle\mathrm{p}\rangle$} & \multirow{2}{*}{$C V_{p}$} & \multicolumn{2}{|c|}{ var vs mean } & \multicolumn{2}{|c|}{ ratio vs mean } & \multicolumn{3}{|c|}{$\operatorname{cov}$} \\
\hline & & & & $\mathrm{q}^{*}$ & $\mathrm{~N}^{*}$ & $q^{*}$ & $\mathrm{~N}^{*}$ & $\mathrm{q}_{1}{ }^{* *}$ & $\mathrm{~N}_{1}^{* *}$ & $\mathrm{~N}_{2}{ }^{* *}$ \\
\hline 500 & 1.25 & 0.5 & 0 & 1.26 & 541 & $1.26 \pm 0.06$ & $517 \pm 260$ & 1.30 & 491 & 618 \\
\hline 500 & 1.25 & 0.5 & 0.1 & 1.26 & 507 & $1.26 \pm 0.07$ & $512 \pm 214$ & 1.26 & 507 & 519 \\
\hline 500 & 1.25 & 0.5 & 0.3 & 1.25 & 464 & $1.26 \pm 0.07$ & $460 \pm 173$ & 1.19 & 537 & 537 \\
\hline 500 & 1.25 & 0.5 & 0.5 & 1.27 & 387 & $1.26 \pm 0.06$ & $403 \pm 131$ & 1.11 & 530 & 563 \\
\hline 500 & 1.25 & 0.5 & 0.7 & 1.28 & 332 & $1.27 \pm 0.06$ & $349 \pm 93$ & 1.02 & 527 & 576 \\
\hline 500 & 1.28 & 0.5 & 0 & 1.26 & 390 & $1.27 \pm 0.06$ & $383 \pm 135$ & 1.24 & 414 & 453 \\
\hline 500 & 1.28 & 0.5 & 0.1 & 1.28 & 382 & $1.28 \pm 0.06$ & $380 \pm 135$ & 1.25 & 406 & 467 \\
\hline 500 & 1.28 & 0.5 & 0.3 & 1.27 & 357 & $1.27 \pm 0.06$ & $360 \pm 120$ & 1.19 & 433 & 433 \\
\hline 500 & 1.28 & 0.5 & 0.5 & 1.31 & 305 & $1.30 \pm 0.06$ & $326 \pm 102$ & 1.15 & 415 & 504 \\
\hline 500 & 1.28 & 0.5 & 0.7 & 1.34 & 240 & $1.32 \pm 0.05$ & $252 \pm 56$ & 0.97 & 460 & 486 \\
\hline
\end{tabular}

Table 1: Summary of simulation results I: $N$ and $q$ estimates obtained from simulated data by fitting a parabola to the variance-mean plot, by fitting a straight line to the variance/mean-mean plot, and from analysis of covariance, variance and mean. In all the simulations the resting occupancy was $\mathrm{p}_{\mathrm{A}}=0.8$. q' $=1$ refers to a situation without quantal size variability, while q' $>1$ refers to the presence of quantal size variability. To distinguish intra- and intersite quanta variability, data from simulations with intrasite quantal variability are underlayed with grey colour in the table. (A) $\mathrm{N}=500$ release sites; different but constant and uniform $\mathrm{p}_{0}$. (B) Different numbers of release sites $\mathrm{N}$; constant and uniform $\mathrm{p}_{0}$ of 0.5 . (C) $\mathrm{N}=500$ release sites, divided into two pools of equal size with different constant release probability. The average $\mathrm{p}_{0}$ is always 0.5 and increasing heterogeneity is introduced by setting the pool probabilities to 0.45 and $0.55,0.35$ and $0.65,0.25$ and $0.75,0.15$ and 0.85 . The corresponding coefficients of variation $\mathrm{CV}_{\mathrm{p}}$ are given in the table. 
parameter $\mathrm{N}^{*}$ from parabolic fits to the variance/mean-mean plots is reasonable in absence of quantal variability and in the presence of intrasite quantal variation in case of homogenous release probabilities. For intersite quantal variation, $\mathrm{N}^{*}$ always underestimates the given $\mathrm{N}$ of the simulations (see Table 1, Table 2), as expected from eq. M15. Progressive increase in heterogeneity of the release probability progressively reduces the determined $\mathrm{N}^{*}$ compared to the given $\mathrm{N}$ of the simulations, as proposed by Silver et al. (1998). The quantal size estimates $\mathrm{q}^{* *}$ from the covariance (see eq. M21) between the first response pair in the train progressively decline with increasing heterogeneity in $\mathrm{p}$, in all other situations they provide a good estimate for q'. The estimates $\mathrm{N}^{* *}$ from the covariance (see eq. M17) yield lower results than the $\mathrm{N}$ given in the simulations in any situation with intersite quantal variation as expected from eq. M18. On the other hand in the absence of quantal variability or in the presence of intrasite quantal variability the estimates display reasonable agreement even in the presence of heterogeneity in the release probability.

A

\begin{tabular}{|c|c|c|c|c|c|c|c|c|c|}
\hline \multirow[t]{2}{*}{$\mathrm{N}$} & \multirow[t]{2}{*}{$q^{\prime}$} & \multirow{2}{*}{$\begin{array}{c}\mathrm{Ca} \\
{[\mathrm{mM}]}\end{array}$} & \multicolumn{2}{|c|}{ var vs mean } & \multicolumn{2}{|c|}{ ratio vs mean } & \multicolumn{3}{|c|}{$\operatorname{cov}$} \\
\hline & & & $\mathrm{q}^{*}$ & $\mathrm{~N}^{*}$ & $\mathrm{q}^{*}$ & $\mathrm{~N}^{*}$ & $\mathrm{q}_{1}{ }^{* *}$ & $\mathrm{~N}_{1}^{* *}$ & $\mathrm{~N}_{2}{ }^{* *}$ \\
\hline 500 & 1 & 2 & $0.99 \pm 0.02$ & $468 \pm 51$ & $0.99 \pm 0.07$ & $470 \pm 255$ & 0.97 & 462 & 498 \\
\hline 500 & 1.25 & 2 & $1.28 \pm 0.02$ & $395 \pm 47$ & $1.28 \pm 0.11$ & $397 \pm 268$ & 1.19 & 493 & 517 \\
\hline 500 & 1.28 & 2 & $1.30 \pm 0.02$ & $302 \pm 26$ & $1.29 \pm 0.10$ & $308 \pm 159$ & 1.18 & 386 & 448 \\
\hline 500 & 1 & 4 & $1.00 \pm 0.01$ & $450 \pm 16$ & $1.01 \pm 0.04$ & $443 \pm 110$ & 0.89 & 593 & 549 \\
\hline 500 & 1.25 & 4 & $1.26 \pm 0.01$ & $472 \pm 24$ & $1.26 \pm 0.05$ & $476 \pm 188$ & 1.18 & 560 & 635 \\
\hline 500 & 1.28 & 4 & $1.28 \pm 0.01$ & $360 \pm 13$ & $1.27 \pm 0.05$ & $370 \pm 109$ & 1.17 & 451 & 400 \\
\hline 500 & 1 & 10 & $0.97 \pm 0.01$ & $501 \pm 11$ & $0.98 \pm 0.02$ & $482 \pm 92$ & 0.85 & 643 & 619 \\
\hline 500 & 1.25 & 10 & $1.26 \pm 0.01$ & $489 \pm 16$ & $1.26 \pm 0.04$ & $489 \pm 154$ & 1.15 & 605 & 590 \\
\hline 500 & 1.28 & 10 & $1.27 \pm 0.01$ & $381 \pm 8$ & $1.28 \pm 0.03$ & $372 \pm 81$ & 1.14 & 464 & 437 \\
\hline
\end{tabular}

B

\begin{tabular}{|c|c|c|c|c|c|c|c|c|c|}
\hline \multirow[t]{2}{*}{$\mathrm{N}$} & \multirow[t]{2}{*}{ q' } & \multirow{2}{*}{$\begin{array}{c}\mathrm{Ca} \\
{[\mathrm{mM}]}\end{array}$} & \multicolumn{2}{|c|}{ var vs mean } & \multicolumn{2}{|c|}{ ratio vs mean } & \multicolumn{3}{|c|}{$\operatorname{cov}$} \\
\hline & & & $\mathrm{q}^{*}$ & $\mathrm{~N}^{*}$ & $\mathrm{q}^{*}$ & $\mathrm{~N}^{*}$ & $\mathrm{q}_{1}{ }^{* *}$ & $\mathrm{~N}_{1}^{* *}$ & $\mathrm{~N}_{2}{ }^{*}$ \\
\hline 500 & 1.25 & 2 & $1.27 \pm 0.02$ & $499 \pm 44$ & $1.28 \pm 0.08$ & $475 \pm 253$ & 1.24 & 550 & 652 \\
\hline 500 & 1.25 & 2 & $1.25 \pm 0.02$ & $447 \pm 33$ & $1.24 \pm 0.07$ & $449 \pm 215$ & 1.25 & 436 & 477 \\
\hline 500 & 1.25 & 4 & $1.28 \pm 0.01$ & $485 \pm 17$ & $1.28 \pm 0.05$ & $496 \pm 176$ & 1.22 & 541 & 711 \\
\hline 500 & 1.25 & 4 & $1.29 \pm 0.01$ & $379 \pm 9$ & $1.29 \pm 0.05$ & $377 \pm 93$ & 1.15 & 471 & 1237 \\
\hline
\end{tabular}

Table 2: Summary of simulation results II: (A) $\mathrm{N}=500$ release sites, divided into two pools of equal size with different probabilities of release $\mathrm{p}_{0}$; facilitating $\mathrm{p}_{0}$ as shown in Fig. 5, at 2, 4, and $10 \mathrm{mM}\left[\mathrm{Ca}^{2+}\right]_{\mathrm{ext}}$. (B) Simulation with $\mathrm{N}=500$ release sites and the three-state release site model as suggested by Trommershäuser (2000) for $2 \mathrm{mM}$ and $4 \mathrm{mM}\left[\mathrm{Ca}^{2+}\right]_{\mathrm{ext}}$, and in presence of intra- or intersite quantal variation. 
The results of some preliminary simulations regarding the process of quantal size reduction during a train of stimuli (see Fig. 25) is discussed below in the discussion of the covariance and correlation data (see p. 69).

\subsection{Fluctuation analysis under control conditions}

\subsubsection{Variance - mean analysis under control conditions}

In order to analyse the strong synaptic depression observed at the calyx of Held synapse, a standard protocol consisting of trains of 5 stimuli at $100 \mathrm{~Hz}$ repeated every $10 \mathrm{~s}$ was applied under control conditions, i.e. at $4 \mathrm{mM}\left[\mathrm{Ca}^{2+}\right]_{\mathrm{ext}}$. In Fig. 9 the results of such an experiment are summarised: Fig. 9A shows example traces indicating the depression of the EPSC amplitudes. The fluctuation of the EPSC amplitudes observed, when the stimulus train was applied repetitively, is displayed in Fig. 9B. The EPSC amplitudes for each response in the train are plotted against record number, along with the leak current and the access resistance $R_{s}$, as parameters of the recording quality. The fluctuation analysis is documented with the variance-mean plot in Fig. 9C, and the variance/mean-mean plot in Fig. 9D. Two categories of EPSC amplitude variance-mean plots were observed. The majority (8 of 10 cases) showed a deviation from a hypothetical parabola for the late, depressed responses to the stimulus train. In two out of 10 experiments a linear relationship between variance and mean was obtained (see Fig. 17). Fig. 9C shows a typical variance-mean plot under control conditions. For demonstrating purposes a parabola passing through the origin can be drawn through the data of first and second response $\left(\mathrm{q}^{*}=24.3 \mathrm{pA} ; \mathrm{N}=\right.$ 695). The data from $3^{\text {rd }}$ to $5^{\text {th }}$ response lay below this parabola. Because the initial slope of the variance versus mean parabola depends on the quantal size (see eq. M12), the observed deviation suggests a quantal size reduction. A linear fit passing through the origin to the data of $3^{\text {rd }}$ to $5^{\text {th }}$ response yielded a quantal size of $\mathrm{q}^{*}=11.1 \mathrm{pA}$. Note that the parabola through the origin and the data of $1^{\text {st }}$ and $2^{\text {nd }}$ response was arbitrary in the sense that the data from the second response could deviate from the true parabola, too. However, its deviation can be expected to be smaller compared to the data from $3^{\text {rd }}$ to $5^{\text {th }}$ response. A similar deviation from the parabola in the variancemean plot was observed by Oleskevich et al. (2000) at the endbulb of Held synapse in rats. For the comparison of the results refer to the discussion, p. 68. 

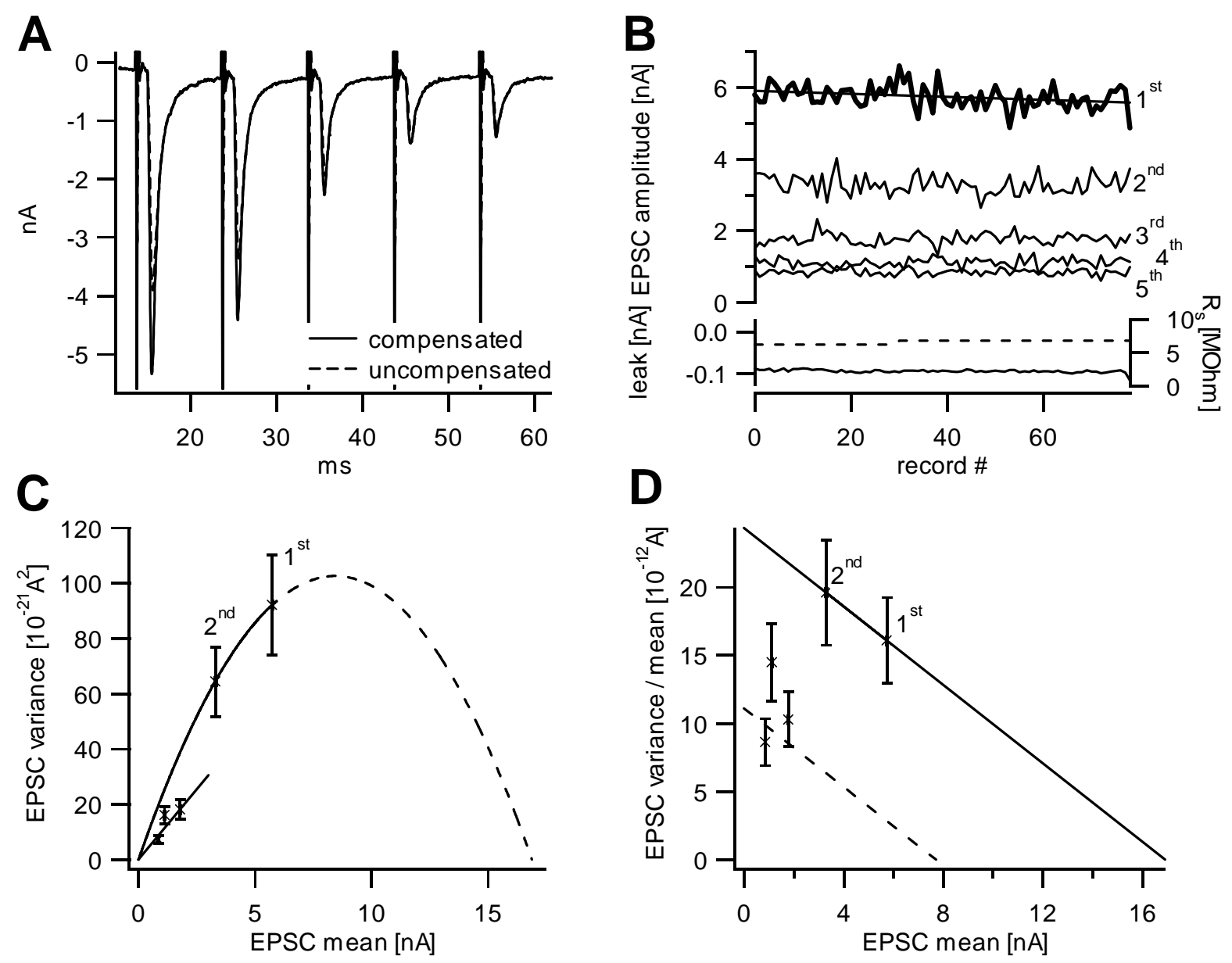

Fig. 9: Non-stationary noise analysis of a recording under control conditions. (A) Example trace before and after offline Rs correction. (B) The plot of the five EPSC amplitudes in the train versus train repetition number $(n=79)$ shows the amplitude fluctuations (long term trend of the first EPSC amplitude: -5.6 \%). Leak current (continuous line) and access resistance $\mathrm{R}_{\mathrm{S}}$ (broken line) are shown in the inset. (C) EPSC amplitude variance-mean plot: Through the data from first and second EPSC a parabola passing through the origin can be drawn $\left(\mathrm{q}^{*}=24.3 \pm 10.1 \mathrm{pA} ; \mathrm{N}^{*}=695 \pm\right.$ 982). The variance for 3 rd to $5^{\text {th }}$ EPSC is below this parabola. A linear fit to the data from 3 rd to $5^{\text {th }}$ EPSC passing through the origin yields $\mathrm{q}^{*}=10.2 \pm 1.2 \mathrm{pA}$. (D) EPSC amplitude variance/mean-mean plot: Trough the data from first and second EPSC a straight line can be placed $\left(\mathrm{q}^{*}=24.3\right.$ and $\left.\mathrm{N}^{*}=695\right)$. The dotted line was determined by offset $\mathrm{q}^{*}=11.1$ (from $\mathrm{C}$ ) and slope $1 / \mathrm{N}^{*}$.

Under control conditions the variance/mean-mean plot (see Fig. 9D) yielded always a scatter of points to which a linear fit yielded a positive slope, whereas the theory predicts a negative slope (see eq. M13). However, as above for the variance versus mean plot, it is shown in Fig. 9D how this could be interpreted. Based on the theory (see eq. M16) the variance/mean-mean plot was expected to give a straight line. Its yaxis intercept is determined by the quantal size $\mathrm{q}^{*}$, and its slope by the negative inverse of the parameter $\mathrm{N}$. Thus, suspecting a reduction in quantal size for the late 
responses, the data points should lie on a family of straight lines of equal slope but with decreasing y-axis intercept. This is shown in Results Fig. 9D. The continuous line passes through the data points of first and second response $\left(\mathrm{q}^{*}=24.3 \mathrm{pA} ; \mathrm{N}=\right.$ 695). The dotted line is a straight line with the same slope as the continuous line, but with $\mathrm{y}$-axis intercept of $\mathrm{q}^{*}=11.1$ taken from the line fit to the data of $3^{\text {rd }}$ to $5^{\text {th }}$ response passing through the origin in Fig. 9C.

Considering the covariance between the amplitudes of consecutive responses in the train, it was now possible to quantify the apparent quantal size reduction, by calculating the quantal size for each compound EPSC during the train.

\subsubsection{Covariance analysis under control conditions}

The amplitudes of consecutive EPSCs in a train displayed negative correlation as expected from the theory (see eq. M16). In Fig. 10 this finding is exemplified for the same experiment as shown in Fig. 9. Fig. 10A demonstrates the correlation between the amplitudes of consecutive EPSCs in a train for selected traces. It is seen that a large EPSC response to the first stimulus in the train is associated with a small EPSC on the second response, and vice versa. The covariance determined for consecutive EPSCs along the train and the calculated quantal size based on this (see eq. M21) is displayed as function of response number Fig. 10B and 10C, respectively. The calculated initial and final quantal sizes are in reasonable agreement with the values from variance analysis in Fig. 9C (24.3 pA and $11.1 \mathrm{pA}$, respectively). The results from the whole data set, as summarized in Fig. 18, demonstrate three important findings. Fig. 18A shows that covariance and correlation between consecutive EPSCs in the train are negative and their absolute values declines from an initial average of $(42.5 \pm 6.4) \times 10^{-20} \mathrm{~A}^{2}(\mathrm{n}=10)$ and $-0.44 \pm 0.06(\mathrm{n}=10)$, respectively, down to about zero with increasing response number. The implication of this is discussed later on. The average numbers for the quantal size $\mathrm{q}^{* *}$, calculated on basis of the covariance measurements on 10 cells, are shown in Fig. 18C. For the first response q** $^{*}$ is $46.7 \pm$ $6.7 \mathrm{pA}(\mathrm{n}=10)$, which is in agreement with the $\mathrm{q}^{\prime}=40.4 \pm 8.6 \mathrm{pA}(\mathrm{n}=4)$ determined from the recording of mEPSCs in other experiments under the same conditions. The apparent decrease in quantal size $\mathrm{q}^{* *}$ with increasing response number is further quantified relative to the initial value in Fig. $18 \mathrm{D}$, showing that the final $\mathrm{q}_{\mathrm{i}}{ }^{* *}$ is reduced to $31.0 \pm 10.0 \%$. Although values for N' could not be obtained, because no 


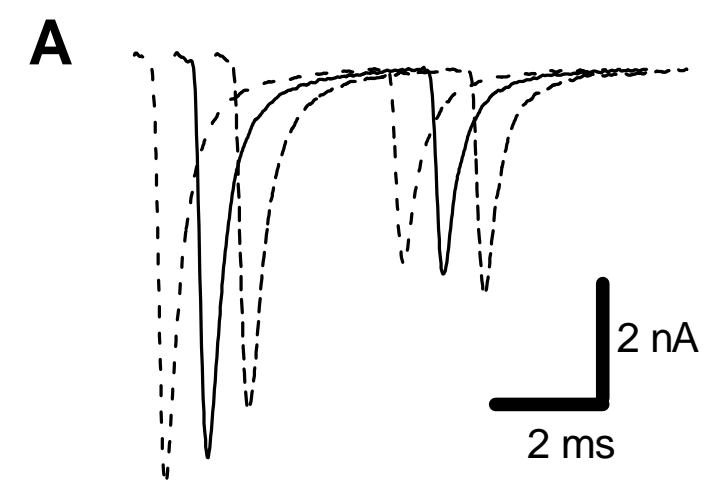

B
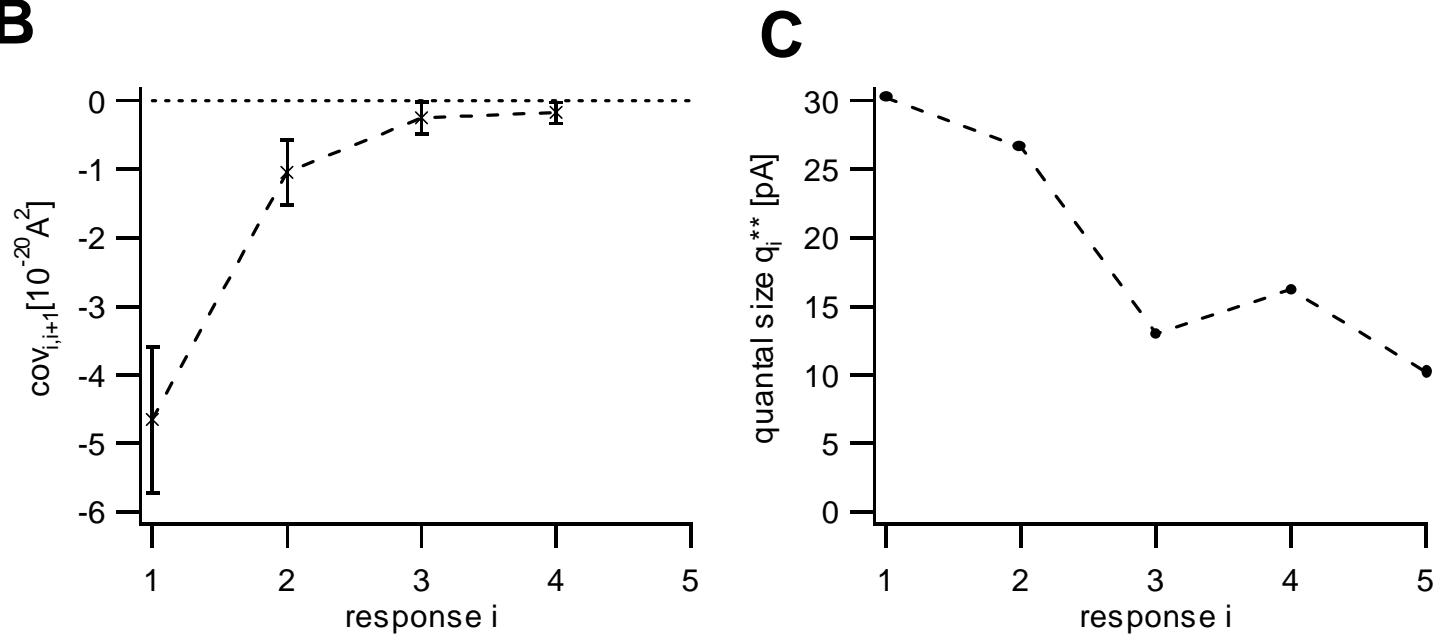

Fig. 10: Covariance analysis example under control conditions (same experiments as in Fig. 9): (A) Example traces showing negative correlation between first and second response in the train. For better visibility, the stimulation artifacts were removed, and the traces shifted along the time axis to different degrees. (B) Plot of covariance between EPSC amplitudes of consecutive responses $i$ and $i+1$ in the train. (C) Calculated $\mathrm{q}_{\mathrm{i}}{ }^{* *}$ (see eq. M21) for each response in the train.

parabolas were available for fitting (note, the parabola shown in Fig. 9C was not fitted, as discussed), $\mathrm{N}^{* *}$ could be determined from the covariance. The data is summarised in Table 4A.

There are several possible reasons for a quantal size reduction operating on the time scale of several ms encountered here. Desensitisation of the postsynaptic AMPA receptors was suggested by Trussel et al. (1993) and Oleskevich et al. (2000) to lead to a quantal size reduction at calyx-type synapses. Desensitisation is defined as a decrease in the response amplitude despite of the ongoing presence of the agonist. In AMPA receptors it proceeds unusually fast with a time constant of $1.82 \pm 0.58 \mathrm{~ms}$ in out-side out patches from neurons of the avian nucleus magnocellularis (Ramann and 
Trussel 1992) and recovers with a time constant of $19 \mathrm{~ms}$ after removal of glutamate (Trussel et al. 1993). To test whether desensitisation was the reason for the observed quantal size reduction, the experiments were repeated in the presence of $100 \mu \mathrm{M}$ cyclothiazide (CTZ), which prevents AMPA receptor desensitisation (Yamada and Rothman 1992, Yamada and Tang 1993, Trussel et al. 1993).

\subsection{Fluctuation analysis in the presence of CTZ}

\subsubsection{Variance - mean analysis in presence of CTZ}

The data discussed in this paragraph was obtained with the standard 5 stimuli at 100 $\mathrm{Hz}$ train protocol repeated every $10 \mathrm{~s}$ in presence of $100 \mu \mathrm{M} \mathrm{CTZ}$ at $4 \mathrm{mM}\left[\mathrm{Ca}^{2+}\right]_{\mathrm{ext}}$. In the presence of CTZ three categories of EPSC amplitude variance-mean plots were observed. Fig. 11 shows the data and analysis of a recording, for which a parabolic variance-mean relationship was obtained. The fit of a parabola passing through the origin to the variance-mean plot (Fig. 11C) gave $\mathrm{q}^{*}=44.9 \mathrm{pA}$ and $\mathrm{N}=391$. A case displaying a linear variance-mean plot is shown in Fig 12. A linear fit passing through the origin gave $\mathrm{q}^{*}=41.9 \mathrm{pA}$ (see Fig. $12 \mathrm{C}$ ). The majority ( 7 of 11 cases) showed a linear variance-mean relationship. In three cases a parabolic relationship according to the theory was observed and in one case a quantal size reduction as discussed above (see Fig. 17). The cases displaying a linear variance-mean plot were typically associated with recordings displaying less depression, transient facilitation or persistent potentiation. Therefore they are believed to be analogous to a subpopulation of cells with a lower initial release probability, as described by Schneggenburger et al. 1999. In these cases only a the initial, linear part of the hypothetical parabola is sampled (Reid and Clements 1999, Clements and Silver 2000).

In the presence of CTZ, in case that the EPSC amplitude variance-mean plot could be fitted with a parabola according to the theory, the variance/mean-mean plot could be fitted with a straight line according to the theory. In the example in Fig. 11D the fit yielded $\mathrm{q}^{*}=(44.3 \pm 7.0) \mathrm{pA}$ and $\mathrm{N}=(399 \pm 96)$ (mean $\left.\pm \mathrm{sdev}\right)$. If the variance-mean plot was fitted by a straight line, the variance/mean-mean plot could be fitted with a straight line according to the theory in some cases. In others the fit gave positive slopes as documented in Fig. 12D or no reasonable straight line fit was feasible at all. 

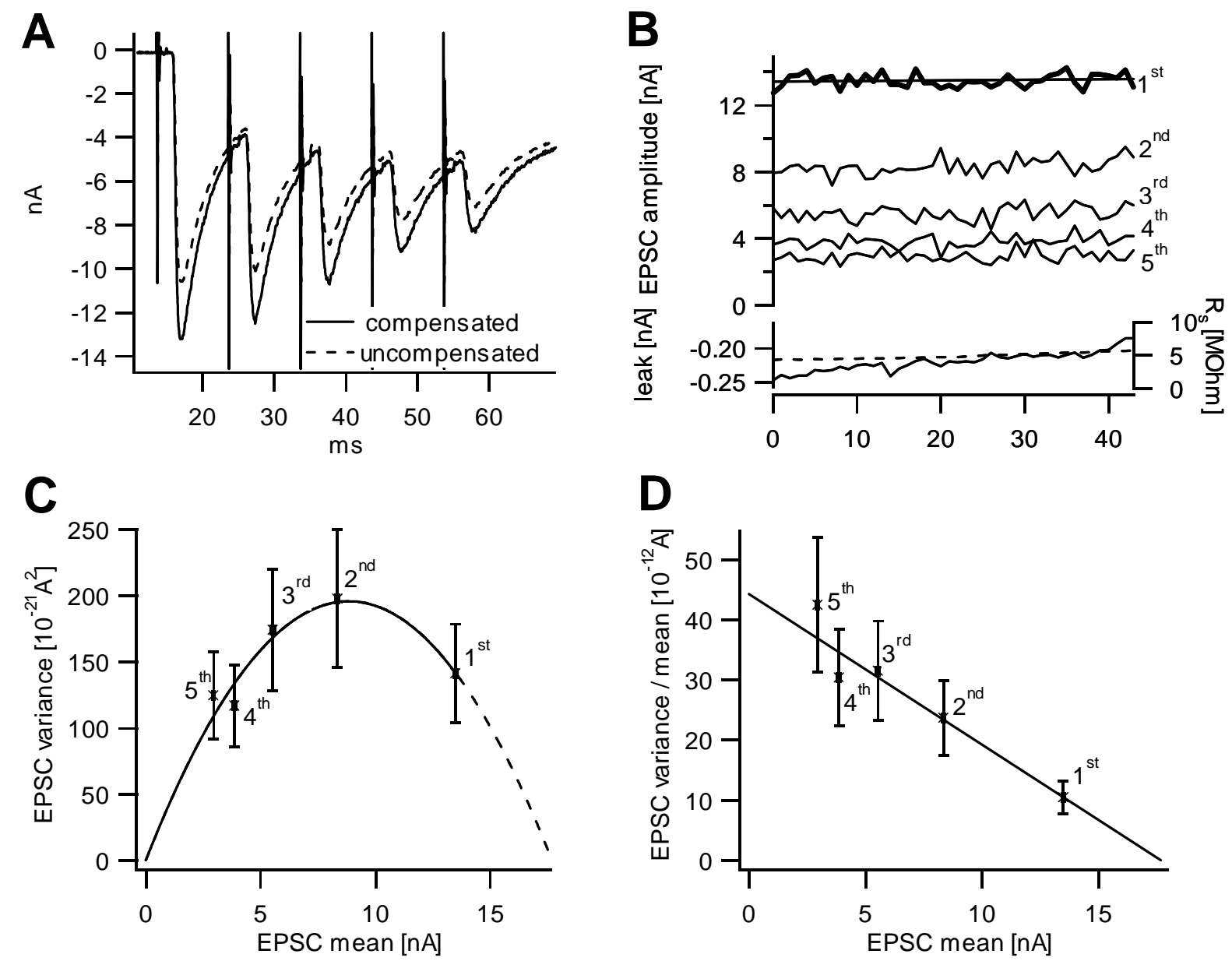

Fig. 11: Non-stationary noise analysis of a recording in the presence of $100 \mu \mathrm{M}$ CTZ; a case of a parabolic EPSC amplitude-mean plot. (A) Example trace before and after offline Rs correction. (B) Plot of the five EPSC amplitudes in the train versus train repetition number $(n=44)$ shows the amplitude fluctuations (long term trend of the first EPSC amplitude: $-1.2 \%$ ). Leak current (continuous line) and access resistance $\mathrm{R}_{\mathrm{s}}$ (broken line) are shown in inset. (C) EPSC amplitude variance-mean plot: The complete data set for all five EPSC can be fitted with a parabola passing through the origin $\left(\mathrm{q}^{*}=44.3 \pm 7.0 \mathrm{pA} ; \mathrm{N}^{*}=399 \pm 96\right)$. (D) EPSC amplitude variance/mean-mean plot: The complete data set for all five EPSC can be fitted with a straight line $\left(\mathrm{q}^{*}=(44.3 \pm 7.0) \mathrm{pA}, \mathrm{N}^{*}=(399 \pm 96)\right.$; mean $\left.\pm \mathrm{sdev}\right)$.

The averages for $\mathrm{q}^{*}$ and $\mathrm{N}$ for the complete CTZ data set obtained from fitting parabolas or straight lines to variance-mean plots were $\mathrm{q}^{*}=31.6 \pm 2.8(\mathrm{n}=11)$ and $\mathrm{N}$ $=896 \pm 241(n=4$; see Table 4B). The results of line fits to the variance/mean-mean plots were always the same as the results from parabola fits to the corresponding variance-mean plots. 
Generally there was no deviation from the parabola or straight line observed in presence of $100 \mu \mathrm{M} \mathrm{CTZ}$, suggesting that AMPA receptor desensitisation is probably a reason for the quantal size reduction observed under control conditions.

A
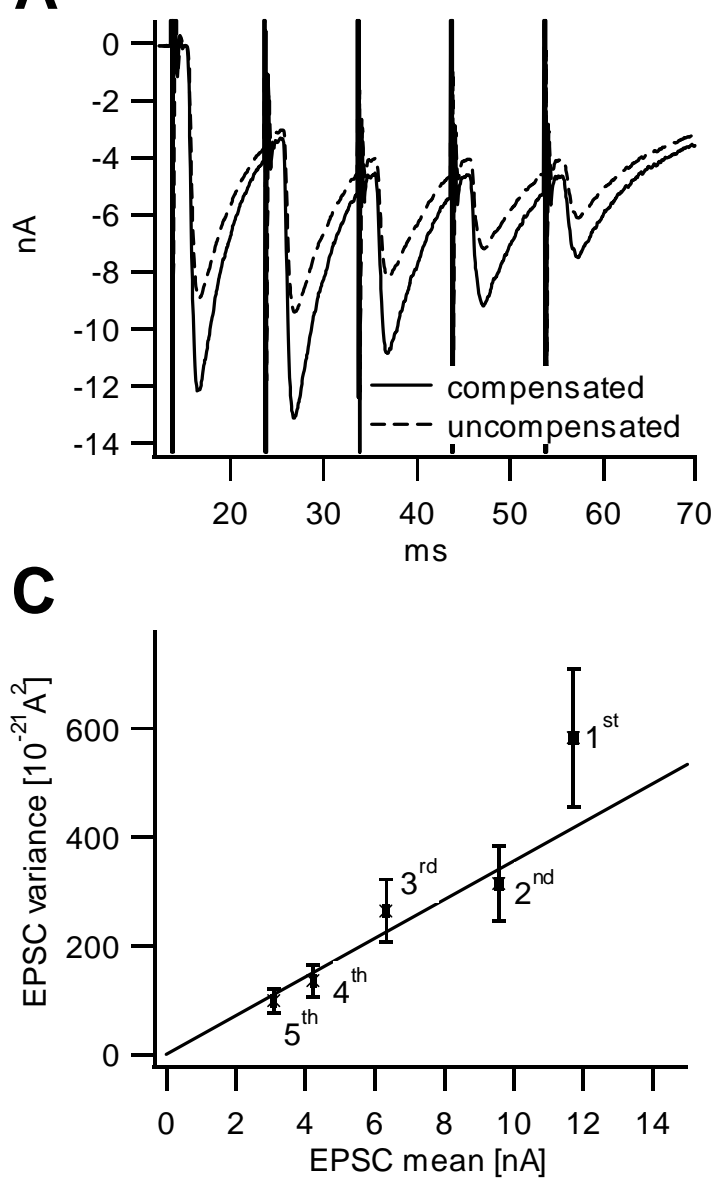

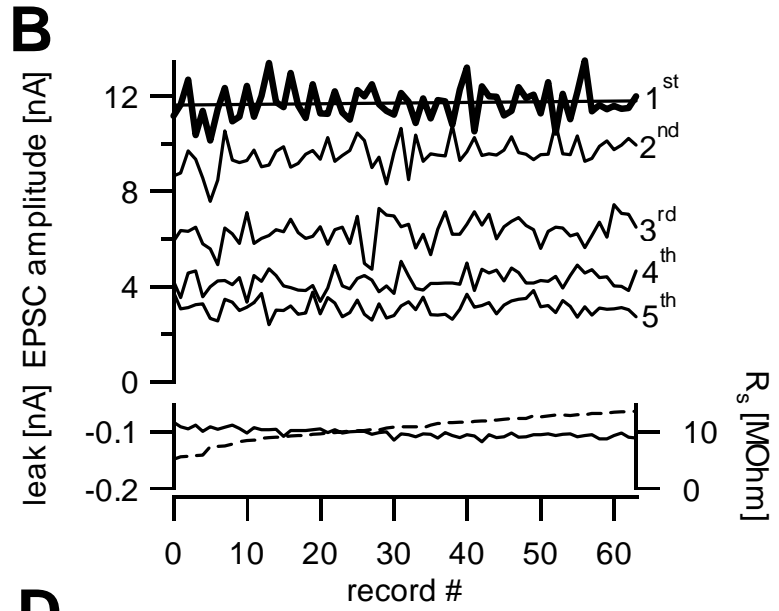

D

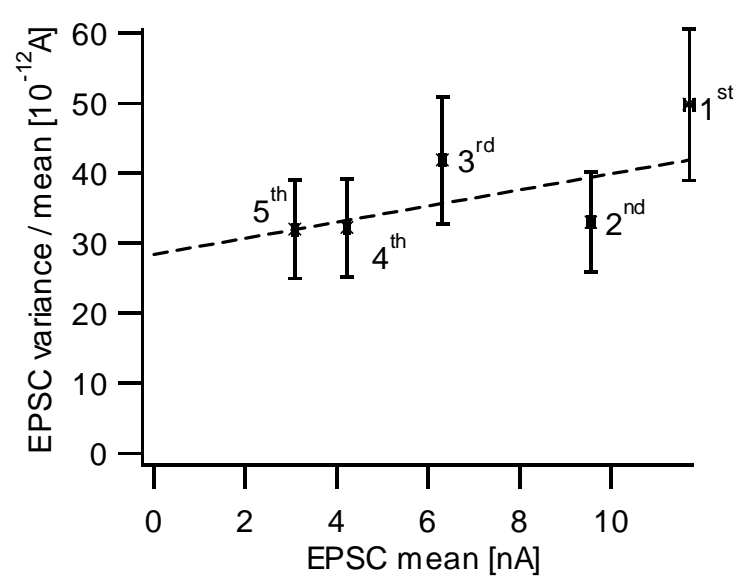

Fig. 12: Non-stationary noise analysis of a recording in the presence of $100 \mu \mathrm{M}$ CTZ; a case displaying a linear EPSC amplitude variance-mean plot. (A) Example trace before and after offline Rs correction. (B) Plot of the five EPSC amplitudes in the train versus train repetition number $(n=64)$ shows the amplitude fluctuations (long term trend of first EPSC amplitude: -1.4\%). Leak current (continuous line) and access resistance $\mathrm{R}_{\mathrm{S}}$ (broken line) are shown in inset. (C) EPSC amplitude variancemean plot: The complete data set for all five EPSC can be fitted with a straight line passing through the origin $\left(\mathrm{q}^{*}=35.6 \pm 3.5 \mathrm{pA}\right.$, mean $\left.\pm \mathrm{sdev}\right)$. (D) EPSC amplitude/mean-mean: The complete data set for all five EPSC can be fitted with a straight, but rising line.

\subsubsection{Covariance analysis in the presence of CTZ}

The covariance analysis in the presence of CTZ, shown in Fig. 13, is from the same experiment as shown in Fig. 11. Again negative correlation is found between 

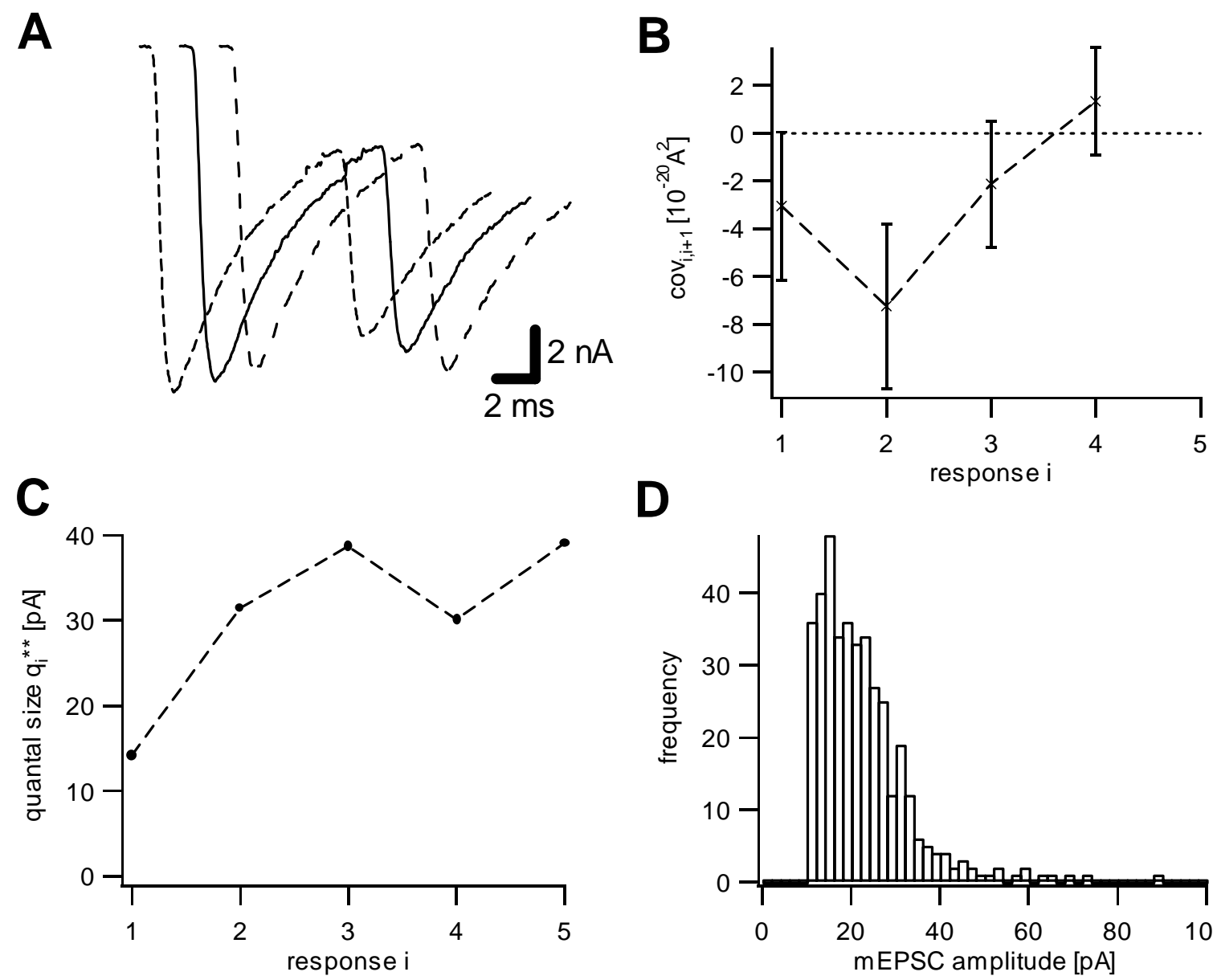

D

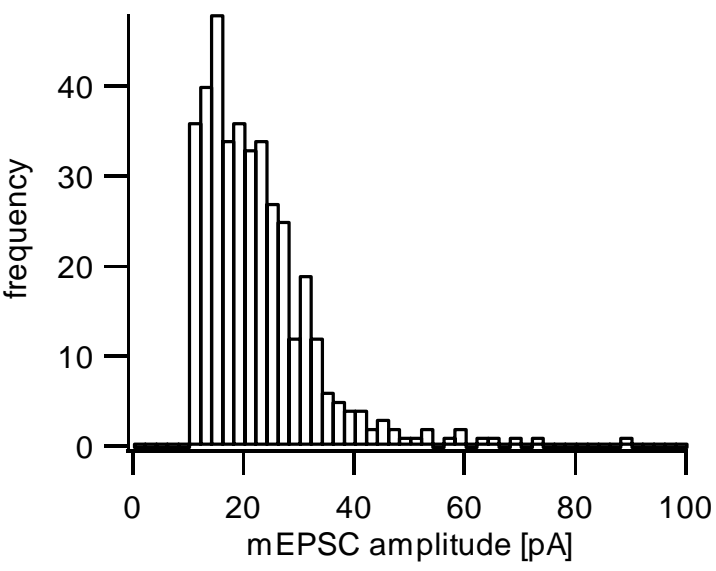

Fig. 13: Covariance analysis examples in CTZ (same experiments as in Fig. 11): (A) Example traces showing negative correlation between first and second response in the train. For better visibility, the stimulation artifacts were removed, and the traces shifted along the time axis to different degrees. (B) Plot of covariance between EPSC amplitudes of consecutive responses $\mathrm{i}$ and $\mathrm{i}+1$ in the train. (C) Calculated $\mathrm{q}_{\mathrm{i}}{ }^{* *}$ (see eq. ) for each response in the train (the low initial $\mathrm{q}^{* *}$ is not a general finding, see Fig. 8 B,C). (D) mEPSC amplitude histogram ( $n=400$ events) yields a quantal size $q$ $=24 \mathrm{pA}$ and the quantal size measure as q' $=38 \mathrm{pA}$, which is in good agreement with the calculated $\mathrm{q}^{* *}$ in $(\mathrm{B})$.

successive EPSCs in the train as demonstrated with selected traces of first and second responses in the train (Fig. 13A). The small covariance for the first response pair compared to the second in Fig. 13B is not a general finding, as shown in the average covariance plot for the whole CTZ data set in Fig. 18A. Consequently the low initial quantal size $\mathrm{q}^{* *}$ in Fig. $13 \mathrm{C}$ is probably an artifact. However the remainder of the calculated quantal size $\mathrm{q}^{* *}$ is almost constant and in agreement with the quantal size determined in other ways. The parabolic fit to the variance- mean plot gave $\mathrm{q}^{*}=44.9$ $\mathrm{pA}$ and from the mEPSC data available in this case (see Fig. 13D) q' $=38.0$ pA was obtained. The summary of the covariance analysis in the presence of CTZ is shown in 
Fig. 18. Initially the covariance in the presence of CTZ is $(47.7 \pm 21.3) \times 10^{-20} \mathrm{~A}^{2}(\mathrm{n}=$ 11) and comparable to the control conditions. Its absolute value decayed similarly as in the control conditions, but not as completely down to zero (Fig. 18A). In contrast the initial correlation coefficient was with $-0.17 \pm 0.05(\mathrm{n}=11)$ less than half times the correlation coefficient obtained under control conditions. The quantal size calculated from the covariance was initially $\mathrm{q}_{1} * *=44.5 \pm 6.7 \mathrm{pA}(\mathrm{n}=11)$ and thus almost the same as under control conditions (see Fig. 10B). This value is also in good agreement with mEPSC data from recordings in the presence of CTZ, which yielded an average of q' $=46.5 \pm 8.5 \mathrm{pA}(\mathrm{n}=4$, Table 4B). The average estimate of the number of release sites $\mathrm{N}^{* *}$ from the covariance is about two times the estimate $\mathrm{N}^{*}$ from the variance mean relationship (see Table 4B). The reason for this discrepancy could be that values of $\mathrm{N}^{* *}>3000$ are outliers. Furthermore the conditions applied here, where in the majority of cases only one half of the parabola is sampled, are not favourable for a determination of the number of release sites $\mathrm{N}$ (Clements and Silver 2000).

The most important finding here was, that even in presence of CTZ, which blocks AMPA receptor desensitisation, there was a reduction in quantal size $\mathrm{q}^{* *}$ to $74 \pm 9 \%$ ( $\mathrm{n}=11$, Fig. 18D) remaining. The most suggestive reasons for this remaining quantal size reduction are postsynaptic receptor saturation and remaining cumulative desensitisation, because CTZ might only slow down desensitisation (Partin et al. 1994), depending the pattern of splice variants expressed in the MNTB principal cells (Geiger et al. 1995). To test for saturation, the combined application of CTZ and 1 $\mathrm{mM}$ kynurenic acid (Kyn) was utilised. Kyn is a reversible AMPA receptor antagonist with fast binding/unbinding kinetics, such that it can be displaced by glutamate during a synaptic response to an extend depending on the amount of glutamate. Consequently this extends the range of glutamate concentrations, which can be sensed below saturation (Diamond and Jahr 1997).

\subsection{Fluctuation analysis in the presence of CTZ and Kyn}

\subsubsection{Variance - mean analysis in CTZ and Kyn}

The data discussed in this and the next paragraph was obtained with the standard 5 stimuli at $100 \mathrm{~Hz}$ train protocol repeated every $10 \mathrm{~s}$ in presence of $100 \mu \mathrm{M} \mathrm{CTZ}$ and 1 

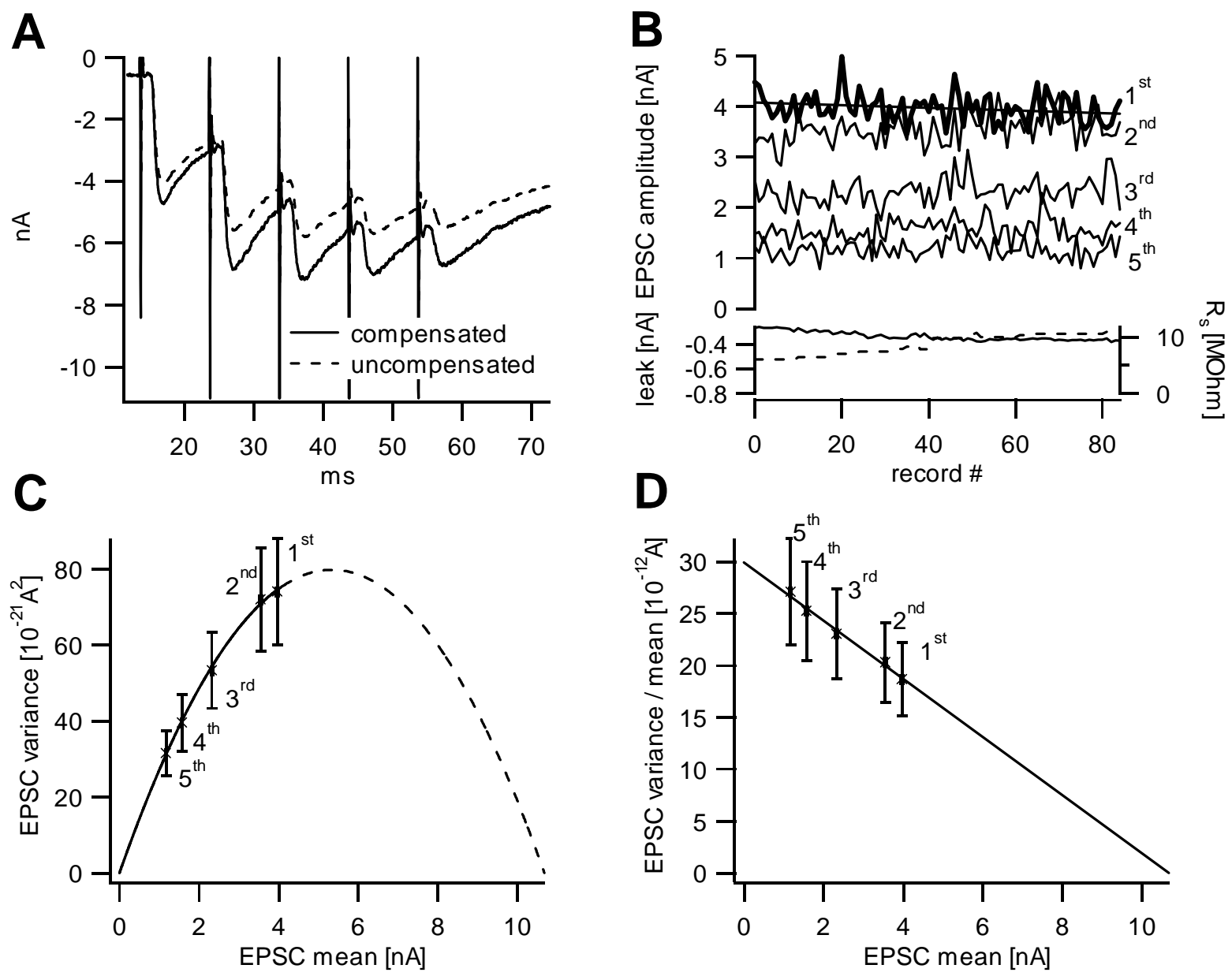

Fig. 14: Non-stationary noise analysis of a recording in the presence of $100 \mu \mathrm{M} \mathrm{CTZ}$ and $1 \mathrm{mM} \mathrm{Kyn;} \mathrm{a} \mathrm{case} \mathrm{which} \mathrm{can} \mathrm{be} \mathrm{fitted} \mathrm{with} \mathrm{a} \mathrm{parabolic} \mathrm{EPSC} \mathrm{amplitude}$ variance-mean relationship. (A) Example trace before and after offline Rs correction. (B) Plot of the five EPSC amplitudes in the train versus train repetition number $(\mathrm{n}=$ 85) shows the amplitude fluctuations (long term trend of the first EPSC amplitude: $5.6 \%)$. (C) EPSC amplitude variance-mean plot: The complete data set for all five EPSC can be fitted with a parabola passing through the origin $\left(\mathrm{q}^{*}=29.9 \pm 5.2 \mathrm{pA} ; \mathrm{N}\right.$ $=357 \pm 222$ ). (D) EPSC amplitude variance/mean-mean plot: The complete data set for all five EPSC can be fitted with a line $\left(\mathrm{q}^{*}=(29.9 \pm 5.3) \mathrm{pA}, \mathrm{N}=(357 \pm 222)\right.$, mean \pm sdev).

$\mathrm{mM}$ Kyn at $4 \mathrm{mM}\left[\mathrm{Ca}^{2+}\right]_{\mathrm{ext}}$. The results are very similar to those obtained for CTZ alone, except that due to the presence of the competitive antagonist Kyn, EPSC amplitudes and their fluctuations were scaled down. As in the presence of CTZ alone, there were three categories of EPSC amplitude variance-mean plots observed under combined application of CTZ and Kyn. An example of a recording yielding data, which could be fitted with a parabola according to the theory, is shown Fig. 14. The fit to the variance-mean plot gave $\mathrm{q}^{*}=29.9 \pm 5.2 \mathrm{pA}$ (mean \pm sdev) and $\mathrm{N}=357 \pm$ 
A
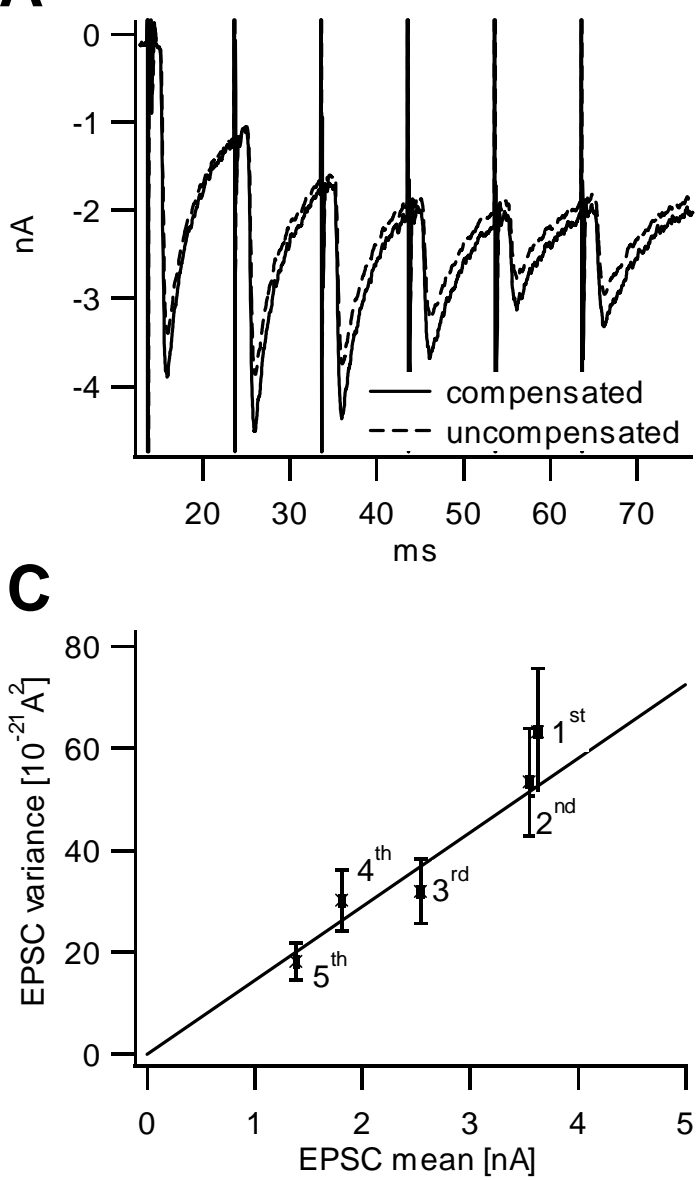

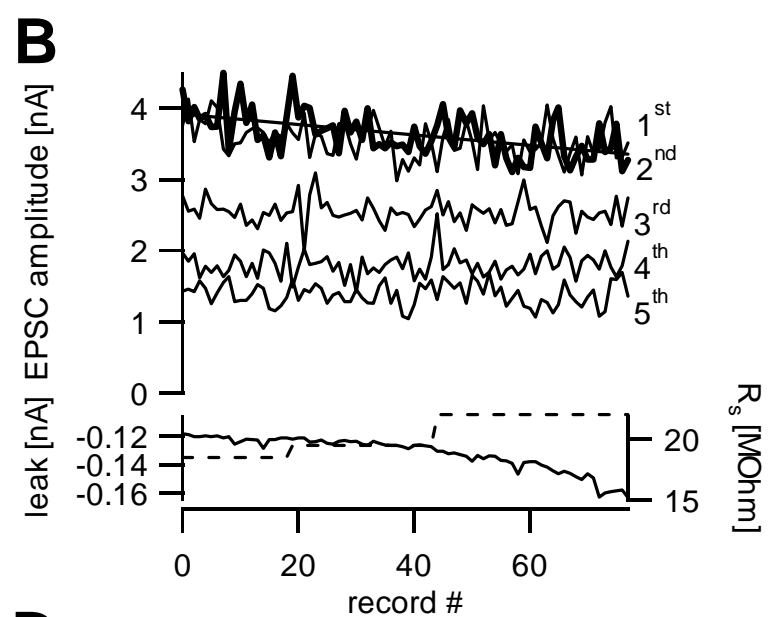

D

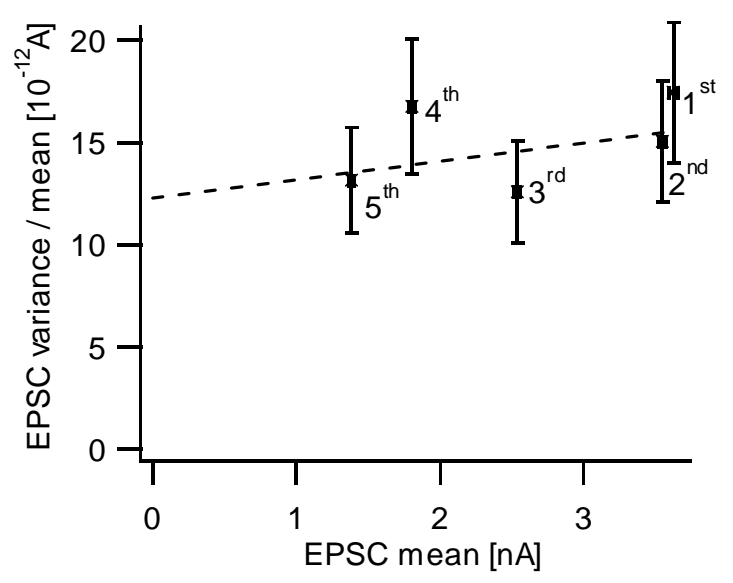

Fig. 15: Non-stationary noise analysis of a recording in the presence of $100 \mu \mathrm{M} \mathrm{CTZ}$ and $1 \mathrm{mM}$ Kyn, which shows a linear EPSC amplitude variance-mean plot. (A) Example trace before and after offline Rs correction. (B) Plot of the five EPSC amplitudes in the train versus train repetition number $(n=77)$ shows the amplitude fluctuations (long term trend of the first EPSC amplitude: -16\%). Leak current (continuous line) and access resistance $\mathrm{R}_{\mathrm{s}}$ (dotted line) are shown in inset. (C) EPSC amplitude variance-mean plot: The complete data set for all five EPSC can be fitted with a line passing through the origin $\left(\mathrm{q}^{*}=14.5 \pm 1.3 \mathrm{pA}\right.$, mean $\left.\pm \mathrm{sdev}\right)$. (D) EPSC amplitude variance/mean-mean plot: The complete data set for all five EPSC can be fitted with a straight, but rising line.

222 (mean \pm sdev, see Fig. 14C). Consequently the variance/mean-mean plot could be fitted with a straight line according to the theory and gave the same parameter values (Fig. 14D). A further example of data from combined application of CTZ and Kyn is shown in Fig. 15. Here the variance-mean plot was fitted with a straight line passing through the origin $\left(\mathrm{q}^{*}=14.5 \pm 1.3 \mathrm{pA}\right.$ (mean \pm sdev), see Fig. $\left.15 \mathrm{C}\right)$. In this case the variance/mean-mean plot can be fitted with a straight but rising line (Fig. 15D). The majority of experiments (6 out of 10) displayed a linear variance-mean plot. Three 
showed a parabolic relationship as expected from the theory and one gave a quantal size reduction as discussed above (see Fig. 17). The results from the line and parabola fits to variance-mean plots for the combined application of CTZ and Kyn are summarised in Table 4C. Again, the results of line fits to the variance/mean-mean plots were always the same as the results from parabolic fits to the corresponding variance-mean plots. The parameter averages were $\mathrm{q}^{*}=22.6 \pm 6.5 \mathrm{pA}(\mathrm{n}=9)$ and $\mathrm{N}=$ $728 \pm 191(n=9)$.

\subsubsection{Covariance analysis under CTZ and Kyn}

The covariance analysis in the presence of CTZ, shown in Fig. 16, is from the same experiment as shown in Fig. 15. Again negative correlation is found between successive EPSCs in the train as demonstrated with selected traces of first and second

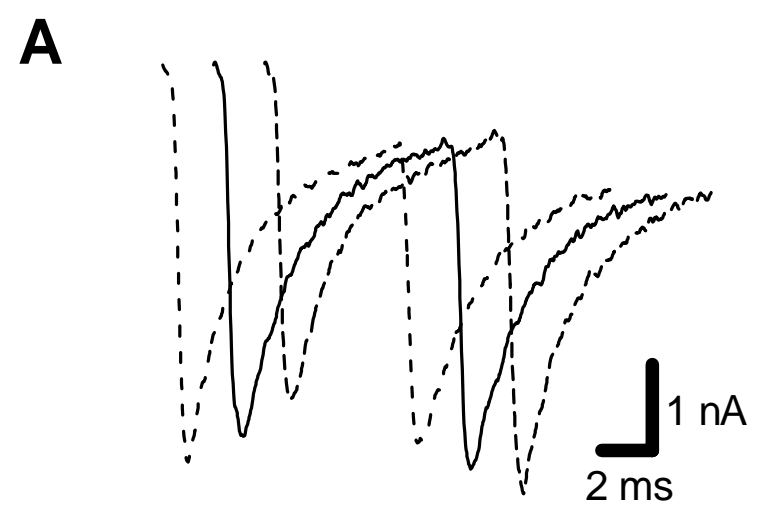

B

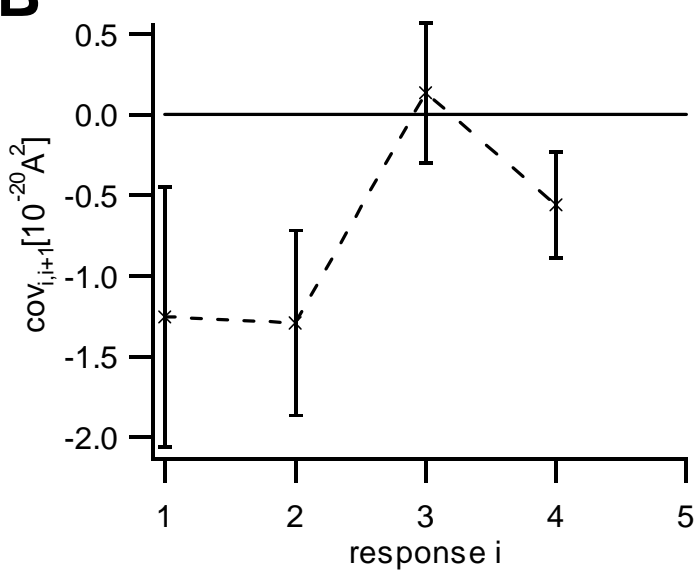

C

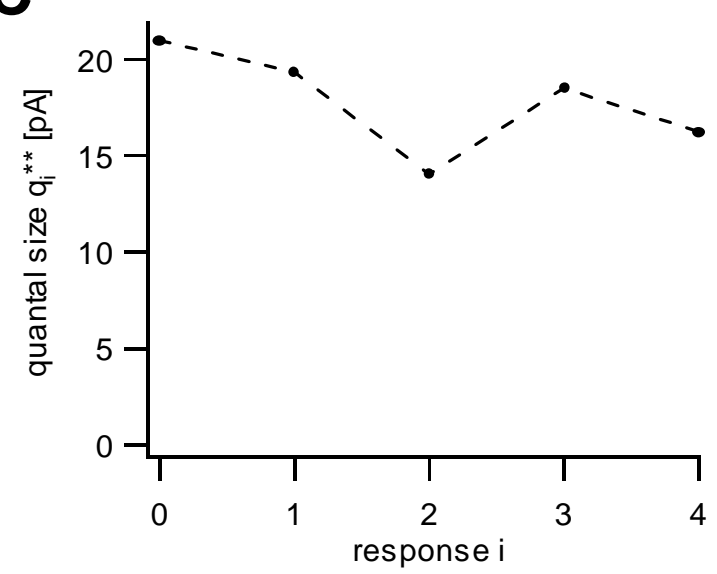

Fig. 16: Covariance analysis example the presence of CTZ combined with Kyn (same experiments as in Fig. 15): (A) Example traces showing negative correlation between first and second response in the train. For better visibility, the stimulation artifacts were removed, and the traces shifted along the time axis to different degrees. (B) Plot of covariance between EPSC amplitudes of consecutive responses i and $\mathrm{i}+1$ in the train. (C) Calculated $\mathrm{q}_{\mathrm{i}}{ }^{* *}$ (see eq. M21) for each response in the train. 
responses in the train (Fig. 16A). The quantal size $\mathrm{q}^{* *}$ calculated from the covariance (see Fig. 16B) is almost constant and in agreement with $\mathrm{q}^{*}=14.5 \pm 1.3 \mathrm{pA}$ (mean \pm sdev) as obtained from the variance-mean plot (see Fig. 15C). The summary of the covariance analysis in the presence of CTZ and Kyn is shown in Fig. 18. The covariance in the presence of CTZ and Kyn is with $(14.8 \pm 12.3) \times 10^{-20} \mathrm{~A}^{2}(\mathrm{n}=9)$ substantially smaller than under control conditions or in the presence of CTZ, as expected from a blocking factor of about $50 \%$ (Neher and Sakaba 2000). Its absolute value decayed similarly as in the control conditions or in CTZ. (see Fig. 18A). The correlation coefficient is further reduced compared to the application of CTZ alone and the control conditions, and not significantly different from zero. The quantal size calculated from the covariance was amazingly constant with $\mathrm{q}_{\mathrm{i}}^{* *}=22.7 \pm 0.3 \mathrm{pA}(\mathrm{n}=$ 9, see Fig. 18C and 18D). This value is also in good agreement with mEPSC data from recordings under control conditions and in the presence of CTZ considering the blocking factor of $50 \%$ (Neher and Sakaba 2000). In one experiment mEPSC data was available because of favourable noise conditions, which gave q' $=21.0$ pA (n = 266), which is in good agreement. The average estimate of the number of release sites $\mathrm{N}^{* *}$ from the covariance is more than two times the estimate $\mathrm{N}^{*}$ from the variance mean relationship (see Table 4C). The reasons for such a discrepancy as discussed in the presentation of the covariance results under CTZ might apply here, too.

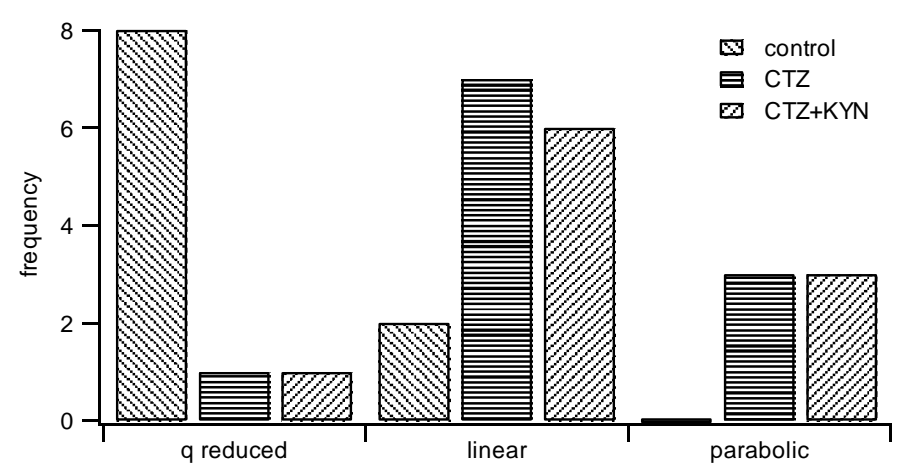

Fig. 17: Summary of variance-mean analysis. This bar diagram shows for how many experiments under the applied conditions the EPSC amplitude variance-mean plot displayed an apparent reduction in quantal size, or can be considered linear or parabolic (control: $\mathrm{n}=10 ; 100 \mu \mathrm{M} \mathrm{CTZ:} \mathrm{n}=11 ; 100 \mu \mathrm{M} \mathrm{CTZ}+1$ mM Kyn: $\mathrm{n}=10$ ). 
A
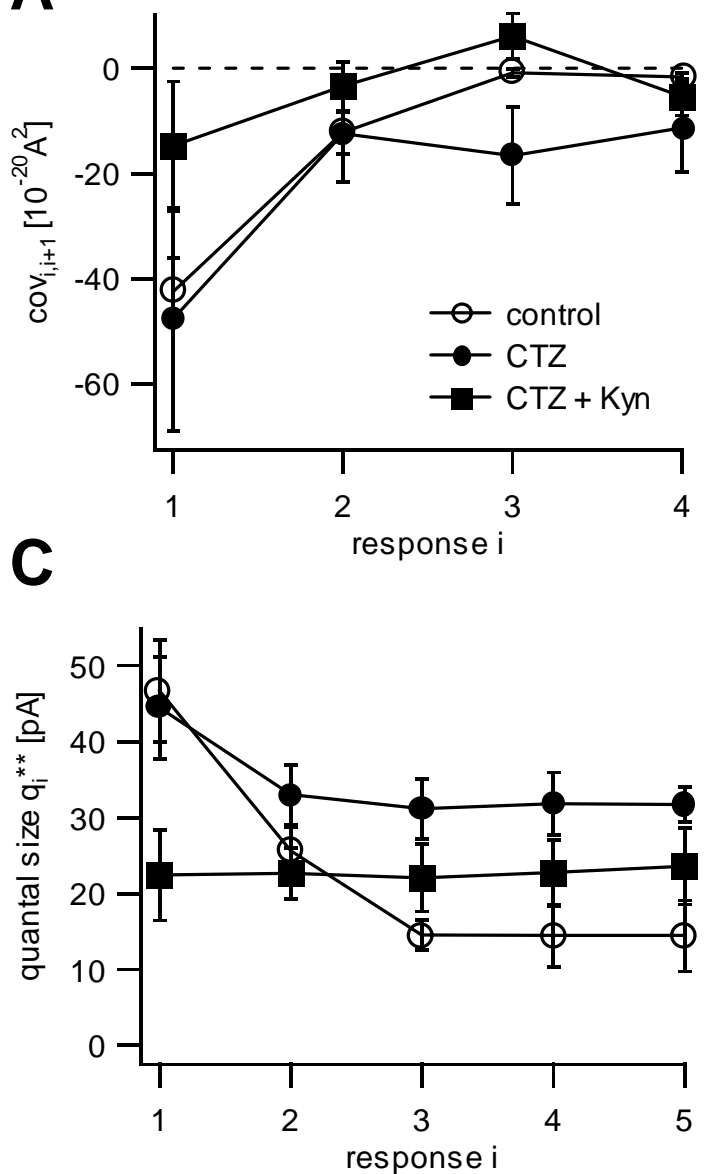

B
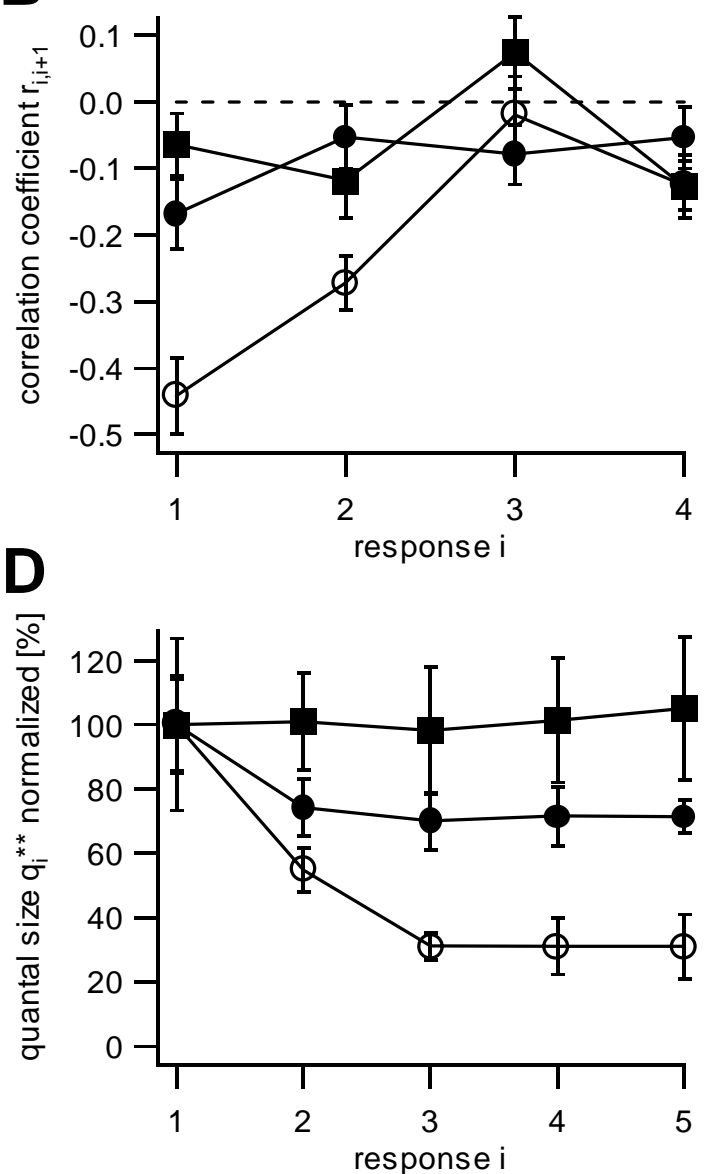

Fig. 18: Summary of the covariance analysis in each applied condition. (A) Plot of the average covariance for each pair of successive responses in the train. (B) Plot of the average correlation coefficient for each pair of successive responses in the train. The correlation coefficient is defined as the ratio of the covariance between two quantities over the product of their standard deviations. (C) Absolute average quantal size $\mathrm{q}_{\mathrm{i}}{ }^{* *}$ as calculated from mean, variance and covariance (see eq. M21). (D) Average quantal size relative to initial average quantal size in the train. This shows that CTZ partially blocks the quantal size reduction observed under control conditions, and additional application of kynurenic acid completely abolishes it (control: $\mathrm{n}=10$; CTZ: $\mathrm{n}=11$; CTZ + Kyn: $\mathrm{n}=10$ ).

\section{A Control}

\begin{tabular}{|c|c|c|c|c|c|}
\hline experiment & $\mathrm{n}$ & $\mathrm{q}[\mathrm{pA}]$ & $\mathrm{CV}_{\mathrm{q}}$ & $\left(1-\mathrm{CV}_{\mathrm{q}}{ }^{2}\right)$ & $\mathrm{q}[\mathrm{pA}]$ \\
\hline $\mathrm{c} 060600 \_01$ & 67 & 34.1 & 0.66 & 1.44 & 49.2 \\
\hline $\mathrm{c060700 \_ 02}$ & 151 & 26.5 & 0.36 & 1.13 & 30.0 \\
\hline c200600_01 & 223 & 41.3 & 0.67 & 1.45 & 60.0 \\
\hline c220600_01 & 238 & 22.4 & 0.30 & 1.09 & 22.3 \\
\hline mean \pm SEM & - & $31.1 \pm 4.2$ & $0.50 \pm 0.10$ & $1.28 \pm 0.10$ & $40.4 \pm 8.6$ \\
\hline
\end{tabular}

\section{B CTZ}

\begin{tabular}{|c|c|c|c|c|c|}
\hline experiment & $\mathrm{n}$ & $\mathrm{q}[\mathrm{pA}]$ & $\mathrm{CV}_{\mathrm{q}}$ & $\left(1-\mathrm{CV}_{\mathrm{q}}{ }^{2}\right)$ & $\mathrm{q}[\mathrm{pA}]$ \\
\hline $\mathrm{c} 010200 \_01$ & 226 & 26.5 & 0.36 & 1.13 & 30.0 \\
\hline c060600_01 & 131 & 54.4 & 0.52 & 1.27 & 69.2 \\
\hline c240200_01 & 399 & 24.0 & 0.75 & 1.56 & 38.0 \\
\hline c280100_01 & 340 & 36.7 & 0.57 & 1.33 & 48.8 \\
\hline mean \pm SEM & - & $35.4 \pm 6.9$ & $0.55 \pm 0.08$ & $1.32 \pm 0.09$ & $46.5 \pm 8.5$ \\
\hline
\end{tabular}

Table 3: Summary of mEPSC data in control conditions (A) and in the presence of CTZ (B). The number of detected events $n$, the average quantal size $q$, the coefficient of variation $(\mathrm{CV})$ of the mEPSC distribution, the factor $\left(1-\mathrm{CV}^{2}\right)$, and the quantal size measure q' are listed. 
A control

\begin{tabular}{|c|c|c|c|c|c|c|c|}
\hline & \multirow[t]{2}{*}{ experiment } & \multicolumn{2}{|c|}{ var or ratio vs mean } & \multicolumn{2}{|c|}{$\operatorname{cov}$} & \multirow{2}{*}{$\begin{array}{c}\text { corrected } \\
\text { cumulative } \\
{[\mathrm{nA}]}\end{array}$} & \multirow{2}{*}{ vesicles } \\
\hline & & $\mathrm{q}^{*}[\mathrm{pA}]$ & $\mathrm{N}^{*}$ & $\mathrm{q}_{1}^{* *}[\mathrm{pA}]$ & $\mathrm{N}_{1}{ }^{* *}$ & & \\
\hline 1) & C040500_01 & - & - & 58.5 & 113 & 20.5 & 438 \\
\hline 2) & C040500_02 & - & - & 22.6 & 348 & 17.8 & 985 \\
\hline 3) & c060700_01 & - & - & 41.8 & 1952 & 37.3 & 1115 \\
\hline 4) & c060700_02 & - & - & 39.3 & 1869 & 37.4 & 1190 \\
\hline 5) & C071099_02 & - & - & 30.2 & 405 & 18.2 & 753 \\
\hline 6) & C160500_02 & - & - & 95.0 & 299 & 24.0 & 316 \\
\hline 7) & C211299_01 & - & - & 53.2 & 385 & 22.6 & 531 \\
\hline 8) & c220600_01 & - & - & 24.8 & 896 & 14.5 & 731 \\
\hline 9) & C281299_01 & - & - & 43.1 & 1006 & 26.8 & 777 \\
\hline 10) & c280100_02 & - & - & 58.6 & 425 & 23.1 & 493 \\
\hline & mean \pm SEM & - & - & $46.7 \pm 6.7$ & $770 \pm 208$ & $24.2 \pm 1.6$ & $733 \pm 11$ \\
\hline
\end{tabular}

\section{B $\quad$ CTZ}

\begin{tabular}{|c|c|c|c|c|c|c|c|}
\hline & experiment & \multicolumn{2}{|c|}{ var or ratio vs mean } & \multicolumn{2}{c|}{ cov } & $\begin{array}{c}\text { corrected } \\
\text { cumulative } \\
{[\mathrm{nA}]}\end{array}$ & vesicles \\
\hline 1) & c010200_01 & $33.1 \pm 5.6$ & $771 \pm 364$ & 81.0 & 645 & 105.1 & 1687 \\
\hline 2) & c130400_02 & $21.0 \pm 5.7$ & $1556 \pm 2810$ & 17.5 & 4140 & 22.0 & 1634 \\
\hline 3$)$ & c151199_01 & $19.5 \pm 2.5$ & - & 43.3 & 1325 & 50.4 & 1513 \\
\hline 4$)$ & c200400_02 & $45.6 \pm 4.7$ & - & 68.1 & - & 66.7 & 1273 \\
\hline 5$)$ & c240200_01 & $44.3 \pm 7.0$ & $399 \pm 96$ & 14.1 & 3683 & 22.1 & 2038 \\
\hline 6$)$ & c261099_01 & $33.6 \pm 3.2$ & - & 60.6 & 656 & 53.9 & 1156 \\
\hline 7$)$ & c280100_01 & $35.6 \pm 3.5$ & - & 58.9 & 1289 & 48.2 & 1064 \\
\hline 8$)$ & c280100_02 & $38.5 \pm 3.5$ & - & 51.2 & - & 43.5 & 1104 \\
\hline 9$)$ & c291099_02 & $30.8 \pm 1.7$ & - & 45.7 & 1706 & 51.1 & 1454 \\
\hline 10$)$ & c291299_01 & $21.0 \pm 4.5$ & $858 \pm 653$ & 17.1 & 1341 & 18.9 & 1437 \\
\hline 11) & c291299_02 & $24.2 \pm 2.2$ & - & 32.0 & 2390 & 34.2 & 1389 \\
\hline & mean \pm SEM & $31.6 \pm 2.8$ & $896 \pm 241$ & $44.5 \pm 6.7$ & $1908 \pm 419$ & $46.9 \pm 3.6$ & $1432 \pm 8$ \\
\hline
\end{tabular}

\section{CTZ+Kyn}

\begin{tabular}{|c|c|c|c|c|c|c|c|}
\hline & \multirow[t]{2}{*}{ experiment } & \multicolumn{2}{|c|}{ var or ratio vs mean } & \multicolumn{2}{|c|}{$\operatorname{cov}$} & \multirow{2}{*}{$\begin{array}{c}\text { cumulative } \\
{[\mathrm{nA}]}\end{array}$} & \multirow{2}{*}{$\begin{array}{c}\text { \# of } \\
\text { vesicles }\end{array}$} \\
\hline & & $\mathrm{q}^{*}[\mathrm{pA}]$ & $\mathrm{N}^{*}$ & $\mathrm{q}_{1}^{* *}[\mathrm{pA}]$ & $\mathrm{N}^{* *}$ & & \\
\hline 1) & c121299_01 & $9.9 \pm 0.7$ & $(949 \pm 2936)$ & 6.1 & 2611 & 6.0 & 776 \\
\hline 2) & c171299_01 & $29.9 \pm 5.2$ & $357 \pm 222$ & 18.7 & - & 12.7 & 544 \\
\hline 3) & c230500_02 & $10.8 \pm 3.2$ & $1129 \pm 2609$ & 8.1 & - & 7.1 & 841 \\
\hline 4) & c250500_01 & $13.7 \pm 1.1$ & - & 19.0 & 4075 & 19.9 & 1859 \\
\hline 5) & c260500_01 & $27.8 \pm 2.1$ & - & 25.3 & 1022 & 19.5 & 898 \\
\hline 6) & c260500_02 & $70.6 \pm 12.9$ & $289 \pm 156$ & 71.9 & 593 & 31.4 & 569 \\
\hline 7) & c301199_03 & $14.5 \pm 1.3$ & - & 21.0 & 1026 & 12.9 & 1139 \\
\hline 8) & c301299_01 & $16.9 \pm 1.6$ & - & 16.4 & - & 16.5 & 1250 \\
\hline 9) & c310500_01 & $9.7 \pm 1.3$ & - & 8.7 & - & 17.0 & 2243 \\
\hline & mean \pm SEM & $22.6 \pm 6.5$ & $728 \pm 191$ & $22.4 \pm 6.0$ & $1865 \pm 651$ & $19.9 \pm 4.6$ & $1124 \pm 14$ \\
\hline
\end{tabular}

Table 4: Summary of noise analysis data in control conditions (A) in the presence of CTZ (B) and with combined application of CTZ and Kyn (C). The columns show q and $\mathrm{N}^{*}$ from variance mean analysis. Omitted $\mathrm{N}$ values indicate that a reasonable parabolic fit was not possible. In that case the given $\mathrm{q}^{*}$ value was obtained from a linear fit. $\mathrm{q}^{* *}$ and $\mathrm{N}^{* *}$ data from the additional analysis of the covariance. The cumulative EPSC amplitudes, in case of control and CTZ alone, were corrected for the apparent quantal size reduction. The number of vesicles associated with the cumulative release were calculated from the ratio of cumulative amplitude over $\mathrm{q}^{* *}$ (for the corrected cumulatives, i.e.control, CTZ) or over q* (CTZ+Kyn). 


\subsection{Synaptic plasticity}

The topic of this study is the analysis of the underlying mechanisms of the strong usedependent depression observed at the calyx of Held synapse. The data and parameters regarding the short-term synaptic plasticity observed under the three different conditions (control, CTZ alone, CTZ combined with Kyn) are summarised Fig. 19 and Fig. 20. Fig. 19A shows the averages of the absolute amplitudes of the EPSCs during the five stimuli train for all three conditions. Fig. 19B displays the averages of the responses normalised with respect to the first response in the train. Under control conditions the mean initial response is $8.35 \pm 1.69 \mathrm{nA}(\mathrm{n}=10)$ and throughout the train the responses are depressed. The second response is on average reduced to $52 \pm 2 \%(\mathrm{n}=10)$ and the $5^{\text {th }}$ response to $12 \pm 3 \%(\mathrm{n}=10)$. In the presence of CTZ transient facilitation is observed on average. The mean initial response is $8.53 \pm 0.74$ $\mathrm{nA}(\mathrm{n}=11)$, the second response is on average facilitated to $110 \pm 10 \%(\mathrm{n}=11)$ and the $5^{\text {th }}$ response is again depressed, but only to $51 \pm 8 \%(\mathrm{n}=11)$. With combined application of CTZ and Kyn the result is very similar to the observed transient facilitation in the presence of CTZ alone. The average absolute initial amplitude is $5.82 \pm 2.08 \mathrm{nA}(\mathrm{n}=9)$. On average the $2^{\text {nd }}$ response is facilitated to $115 \pm 11 \%(9)$ and the $5^{\text {th }}$ response is depressed to $55 \pm 12 \mathrm{nA}(\mathrm{n}=9)$.

The relationship of $2^{\text {nd }}$ and $5^{\text {th }}$ EPSC amplitude to the $1^{\text {st }}$ EPSC amplitude of the train in the individual cells is shown in Results Fig. 20A, B. Under control conditions (open circles) the $2^{\text {nd }}$ EPSC amplitude data lies below the unity line, only in the low $1^{\text {st }}$ amplitude range two out of 10 data points are on the line. In contrast with application of CTZ alone (filled circles) or in combination with Kyn (filled squares) the $2^{\text {nd }}$ EPS amplitude data scatters around the unity line for the complete range of $1^{\text {st }}$ EPSC amplitudes (Fig. 20A). The $5^{\text {th }}$ EPSC amplitude data lies far below the unity line under control conditions. In presence of CTZ alone and with combined application of CTZ and Kyn the majority of the amplitude data of the $5^{\text {th }}$ EPSC is below the unity line, while a considerable number is still on or near the unity line (Fig. 20B). There is a correlation between the cumulative EPSC amplitude of the five stimulus train and the mean EPSC amplitude of the $1^{\text {st }}$ response (see Fig. 20C). For control data a line fit yields an offset of $5.7 \mathrm{nA}$ and a slope of 1.11. The data for CTZ and the combination of CTZ with Kyn could be fitted together with a line and 
A
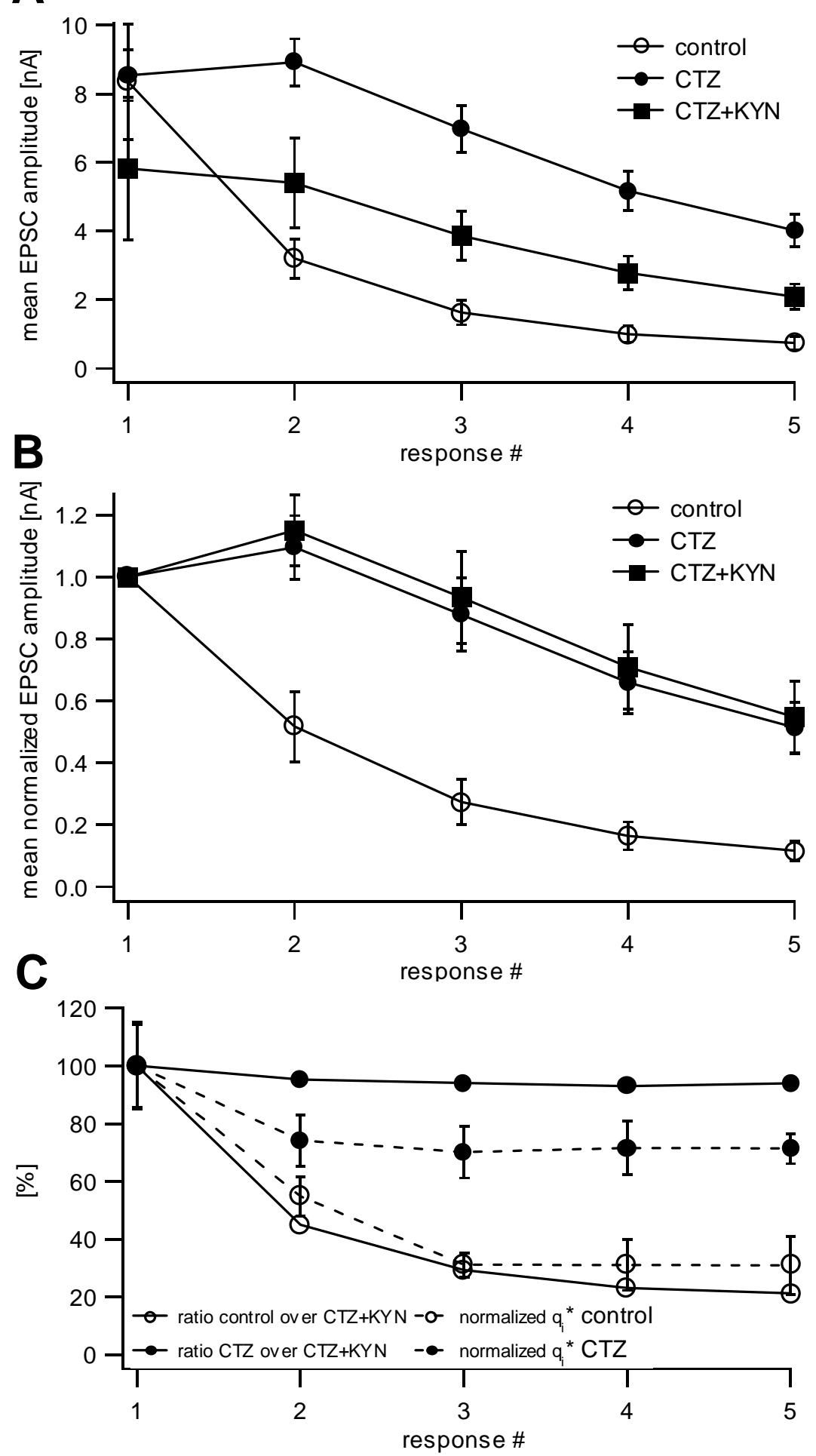

Fig. 19: Summary of synaptic short-term plasticity in 5 stimuli trains at $100 \mathrm{~Hz}$. (A) Average absolute EPSC amplitudes under each condition. (B) Average normalized EPSC amplitudes under each condition. Averaging was performed after normalization with respect to first EPSC amplitude in a train. (control: $\mathrm{n}=10$; CTZ: $\mathrm{n}=11$; CTZ + kyn: $\mathrm{n}=9$ ). (C) Ratios the of normalized EPSC amplitudes under control conditions and in the presence of CTZ over the normalized EPSC amplitudes in the presence of CTZ and Kyn (see (B)) and comparison with normalized quantal size $\mathrm{q}_{\mathrm{i}}{ }^{* *}$ as calculated from the covariance (see Fig. 18C). For interpretation refer to text. 

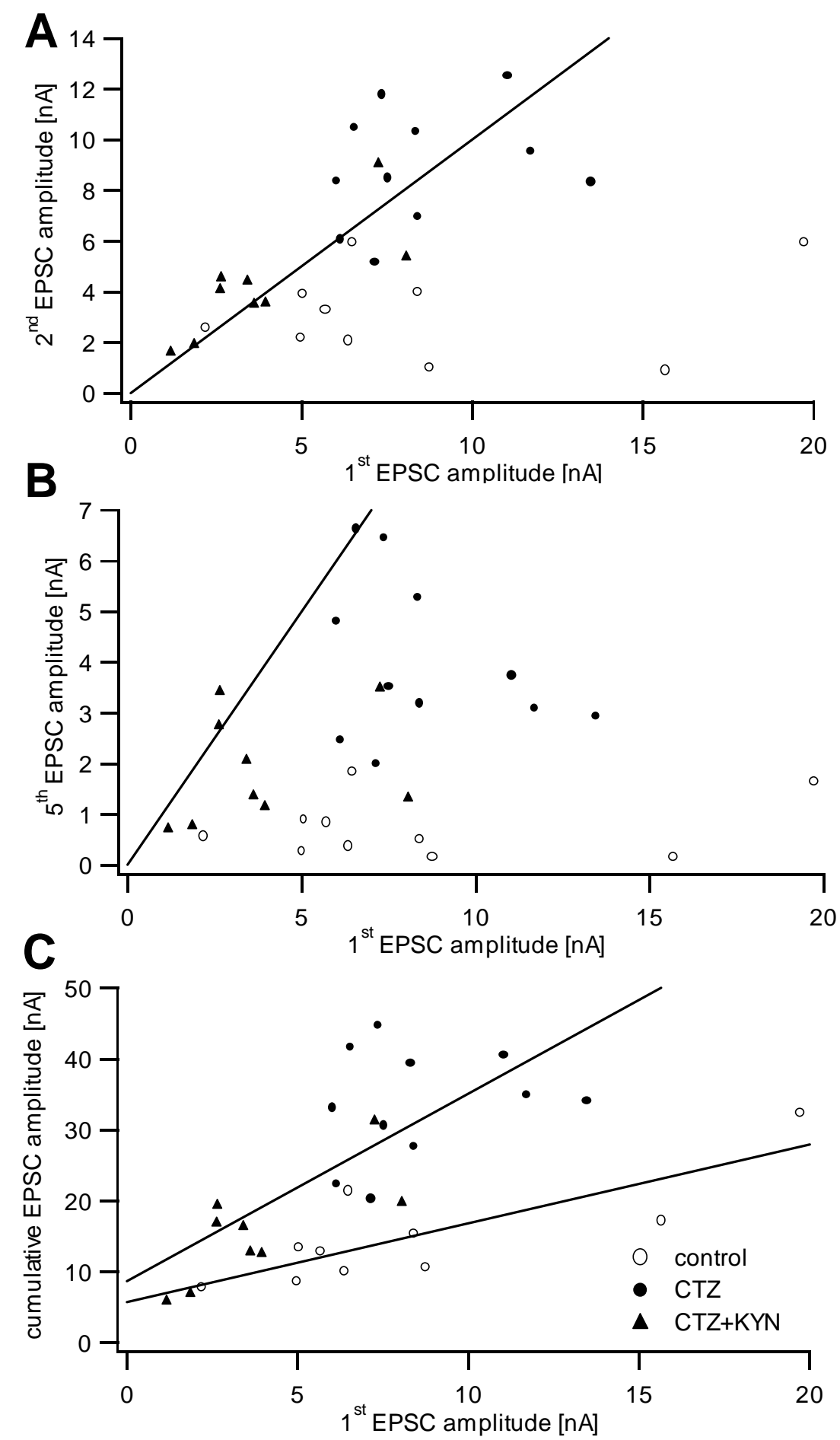

Fig. 20: Summary of plasticity behaviour under control conditions, in the presence of CTZ alone and with combined application of CTZ and Kyn. (A) A plot of $2^{\text {nd }}$ versus $1^{\text {st }}$ EPSC amplitude shows that under control conditions the majority of cells displayed depression already at the second response, while in the presence of CTZ alone and in combination with Kyn, cells show little or no depression and some even facilitate. (B) The plot of $5^{\text {th }}$ versus $1^{\text {st }}$ EPSC amplitude displays the strong depression under control conditions. Compared to control, cells exhibited less depression in the presence of CTZ alone and with Kyn, and in some cases no depression at all. (C) Plotting the cumulative response amplitude versus EPSC1 shows a clear correlation. Under application of CTZ alone and with Kyn the relationship was steeper (offset $=8.6 \mathrm{nA}$; slope 2.6) compared to control conditions (offset $=5.7 \mathrm{nA}$; slope $=1.11$ ). 
yielded a similar offset of $8.6 \mathrm{nA}$, but a more than two times larger slope of 2.6, as expected if desensitisation and saturation reduce the responses following the first in the train and consequently lower the cumulative amplitude under control conditions.

In summary application of CTZ alone and in combination with Kyn uncovered a transient facilitation, followed by depression. The depression was less compared to control conditions. However, under conditions preventing postsynaptic receptor desensitisation and saturation depression was persistent.

A

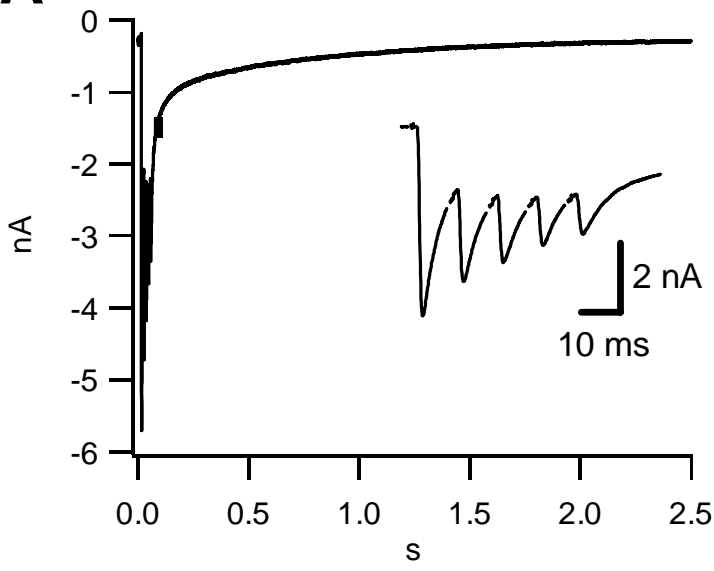

C

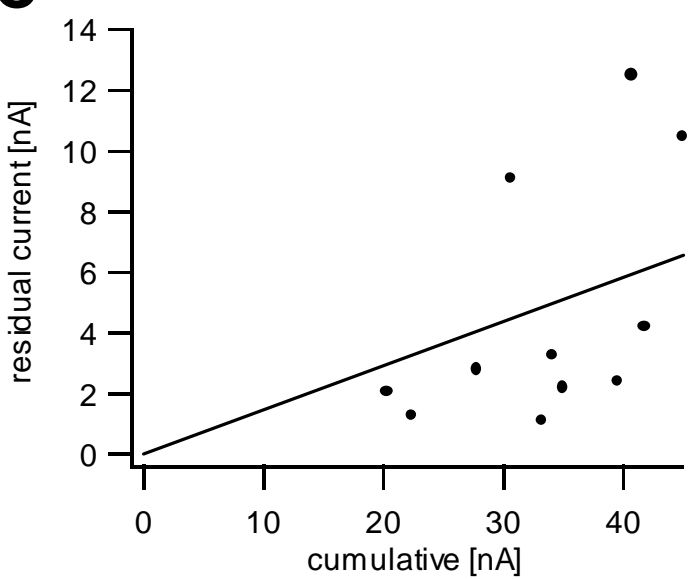

B

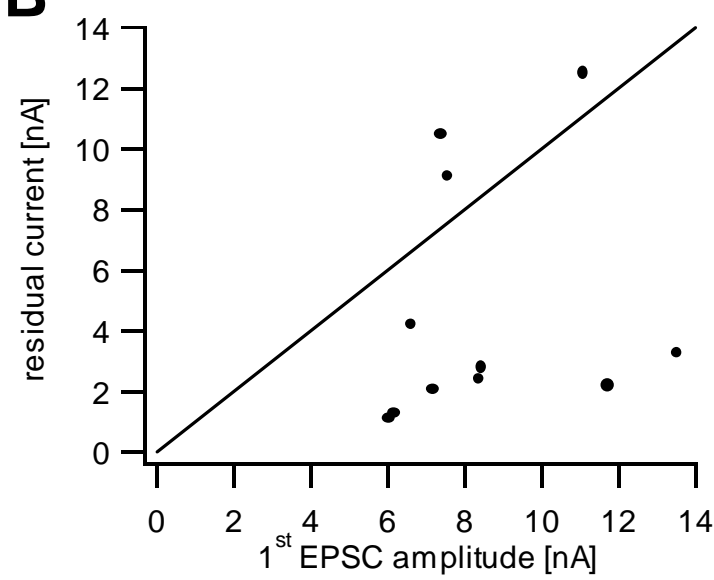

D

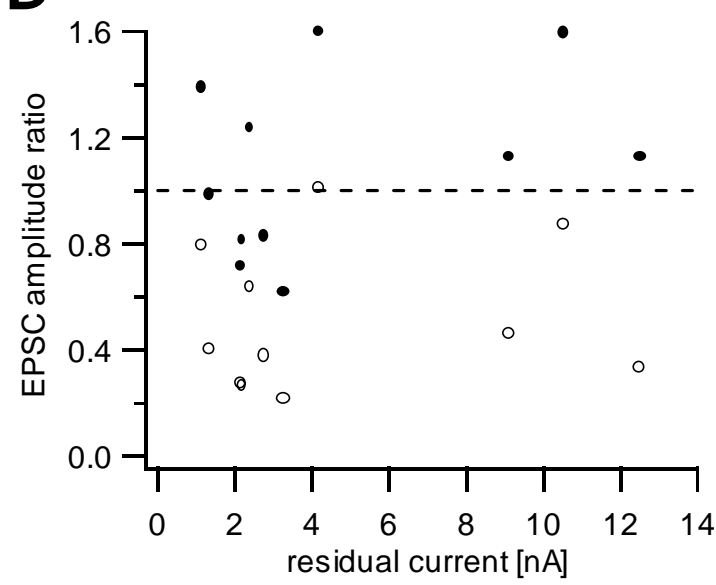

Fig. 21: Analysis of the residual current in the presence of CTZ. (A) The plot of an example trace shows the slow decay of the residual current after a five stimulus train at $100 \mathrm{~Hz}$. The inset displays a blow up of the EPSC train. To quantify the residual current the mean current over a $2 \mathrm{~ms}$ interval $30 \mathrm{~ms}$ after the last stimulus in the train was determined. (B) The plot of residual current versus $1^{\text {st }}$ EPSC amplitude shows little correlation between these parameters. (C) The residual current shows some dependence on the cumulative EPSC amplitude. (D) The plots of the ratios of $2^{\text {nd }}$ to $1^{\text {st }}$ EPSC amplitude and $5^{\text {th }}$ to $1^{\text {st }}$ EPSC amplitude versus residual current show that there is no dependence of short-term synaptic plasticity on the amount of the residual current. 


\subsection{Residual current and EPSC amplitude versus charge relationship}

Application of CTZ prolongs the EPSC decay. During the five stimuli train this leads to EPSC overlap and consequently to a considerable build-up of background current, also referred to as residual current (Otis et al. 1996b) (see Fig. 21A). To quantify the residual current the average current over a $2 \mathrm{~ms}$ interval $30 \mathrm{~ms}$ after the last stimulus was taken (see Fig. 21A). There seems to be little if any correlation between the $1^{\text {st }}$ EPSC amplitude in the five stimuli train and the residual current (see Fig. 21B). In contrast there is some correlation between the residual current and the cumulative EPSC amplitude of the five stimuli train (see Fig. 21C). If the residual current results from the glutamate concentration in the synaptic cleft at the end of the train, then one would expect, that it is dependent on the total amount of release, and not only on the first response. Synaptic plasticity and the amount of residual current appear uncorrelated (Fig. 21D).

In Fig. 22A example traces of isolated EPSCs of different amplitude are shown under control conditions and in the presence of CTZ for the same cell. Fig. 22B shows that EPSC peak and EPSC charge are proportional under control conditions. This is in agreement with the suggestion by Borst and Sakmann (1996) that the distribution of quanta release per time scales uniformly in making up a multi-quantal EPSC of a certain size. The proportionality factor relating EPSC amplitude and charge is $0.49 \mathrm{~ms}^{-1}$ for control conditions (see Fig. 22B). In the presence of CTZ the EPSC peak amplitude and charge are still linearly related, but a considerable charge offset is obtained at zero amplitude (see Fig. 22B). The offset was determined to be $-2.42 \mathrm{nA}$, and the slope was $116 \mathrm{~ms}^{-1}$. According to Borst and Sakmann (1996) the offset should not be due to asynchronous release uncovered by inhibition of desensitisation, because its size is constant and therefore does not correlate with the amount of synchronous release. As mentioned above it should rather be the result of the so called residual current arising from build-up of the released glutamate in the synaptic cleft (Trussel et al. 1993, Otis et al. 1996b). For the implications of this regarding the analysis, and the question whether the EPSC amplitude or the EPSC charge is the appropriate parameter to quantify release, refer to the discussion (p. 63). 
A
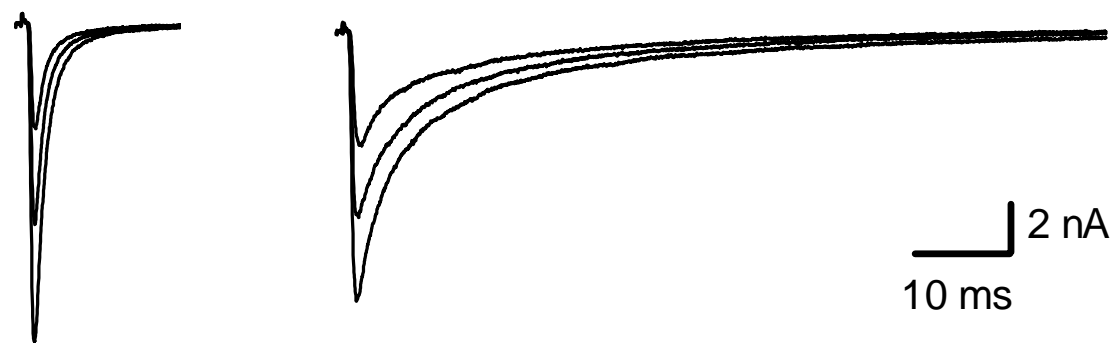

B

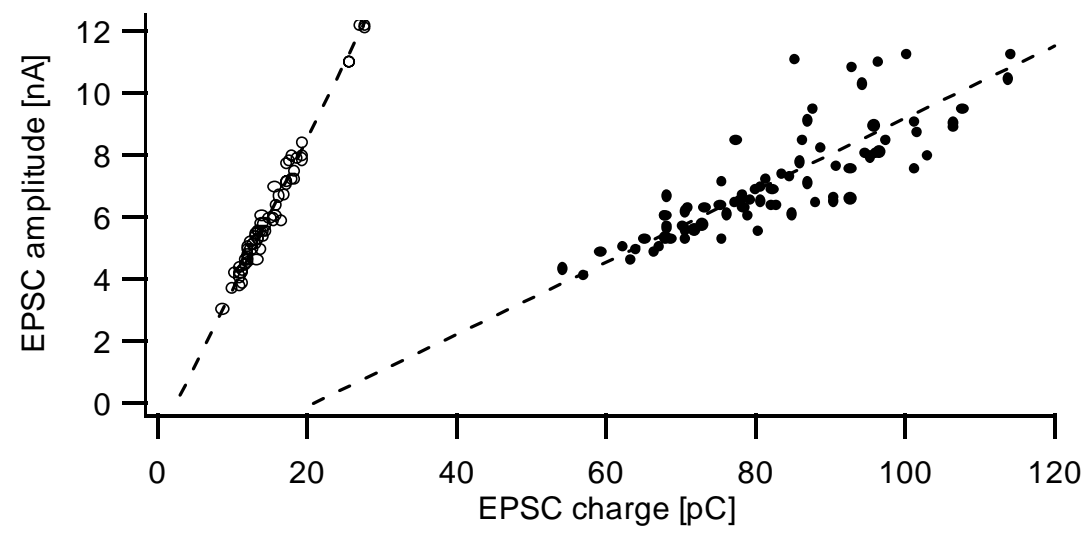

Fig. 22: EPSC amplitude versus charge relationship. (A) Example EPSCs of different amplitude in the same cell in the absence and the presence of CTZ obtained from stimulus trains at $10 \mathrm{~Hz}$, which cause depression without response overlap. (B) Plot of EPSC amplitude versus EPSC charge under control conditions (open circles) and in the presence of CTZ (filled circles). A linear fit to the control data yielded an offset of $-1.22 \mathrm{nA}$ and a slope of $0.49 \mathrm{~ms}^{-1}$. The CTZ data gave an offset $=-2.42 \mathrm{nA}$ and slope $=0.12 \mathrm{~ms}^{-1}$.

\subsection{Cumulative EPSC amplitudes}

Estimates of the size of the pool of release competent vesicles can be made by integrating the release evoked by a train of stimuli and correcting for refilling in between stimuli (Elmquist and Quastel 1965, Schneggenburger et al. 1999). Such a pool estimate can be considered a lower limit of the number of release sites present. The protocol for this type of pool estimation consisted of trains of 21 stimuli at 100 $\mathrm{Hz}$ repeated every $40 \mathrm{~s}$ in order to allow complete recovery in-between trains. Fig. 23A shows average EPSC traces in one cell under control conditions and in the presence of CTZ. Fig. 23B displays the cumulative EPSC amplitudes under both conditions in the same cell. It illustrates the determination of the pool estimate as the $y$-axis intercept of a linear fit to the uniformly rising phase of the cumulatives between stimulus 10 and 21 . The average pool estimate under control conditions in 
terms of current was $13.2 \pm 2.6 \mathrm{nA}(\mathrm{n}=6)$ and in the presence of CTZ it was $31.4 \pm$ $6.1 \mathrm{nA}(\mathrm{n}=6)$. Applying the average quantal size values $(31.1 \pm 4.2 \mathrm{pA}$ in control; $35.4 \pm 6.9 \mathrm{pA}$ in CTZ; see Table 4) from independently recorded mEPSC data the pool estimates could be expressed as numbers of vesicles. This yielded $424 \pm 84$ ( $\mathrm{n}=$ 6) vesicles in control and $887 \pm 119(n=6)$ vesicles in the presence of CTZ. In the

A

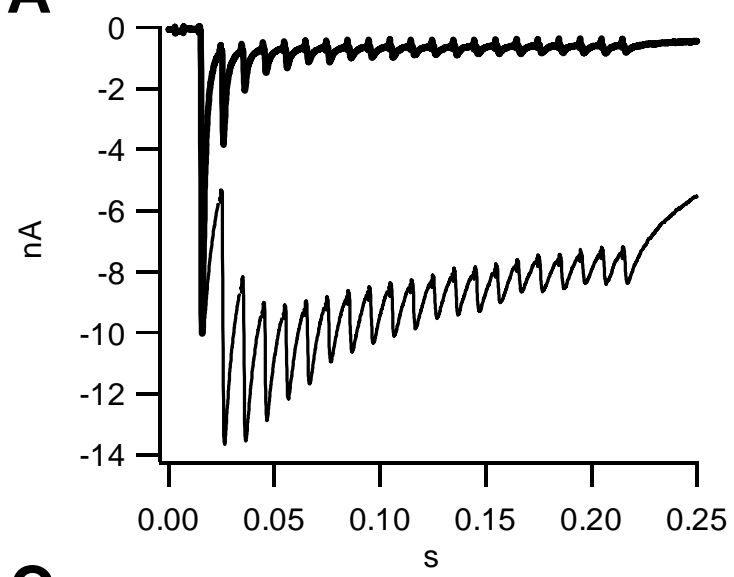

C

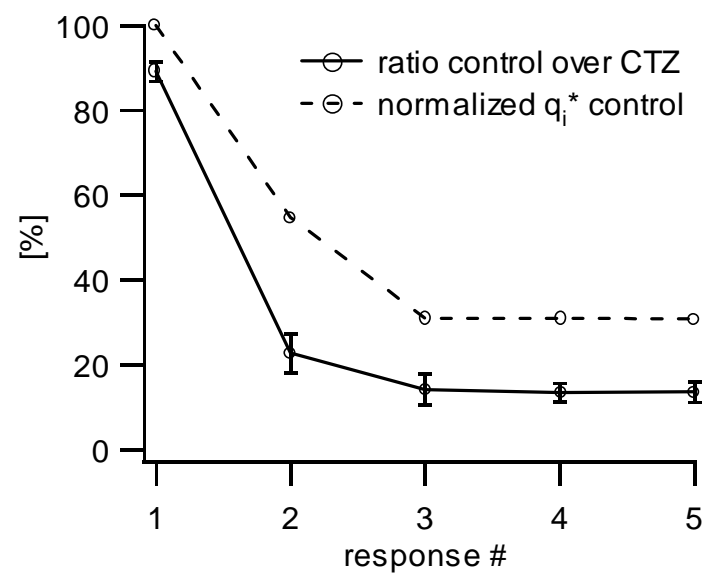

B
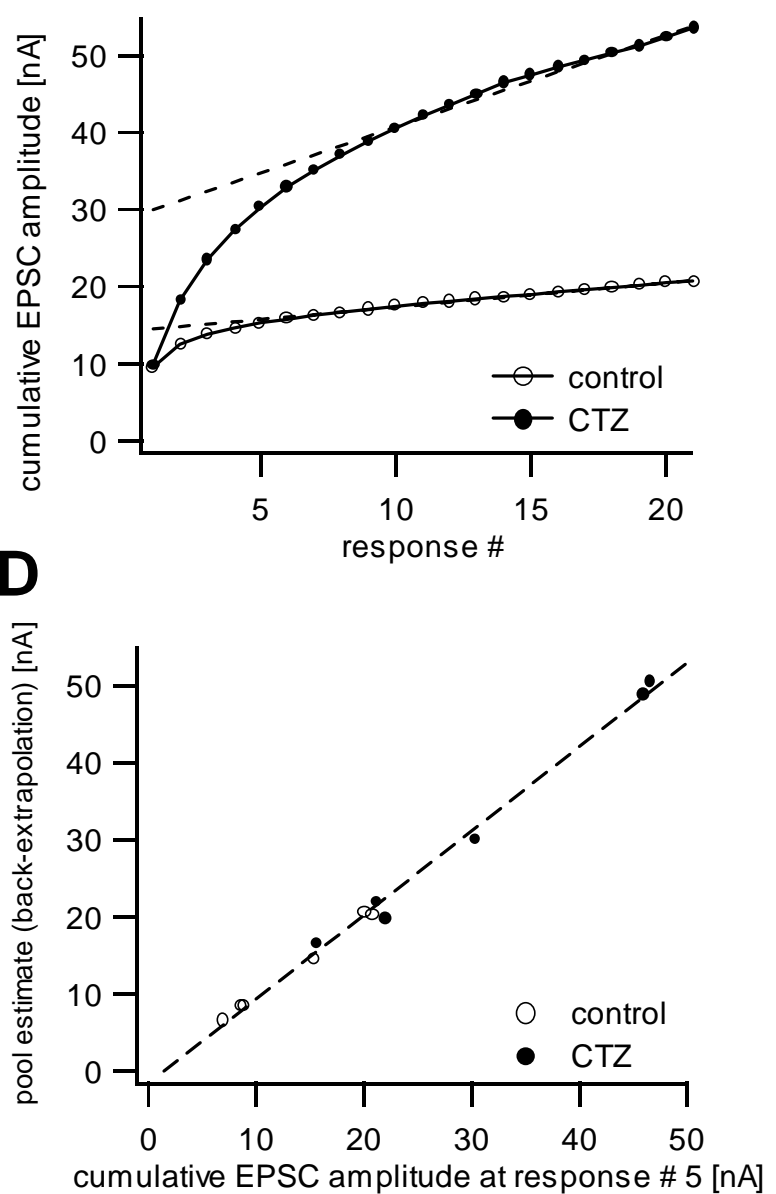

Fig. 23: Pool size estimate from the cumulative EPSC amplitudes in the absence and presence of CTZ from trains with 21 stimuli at $100 \mathrm{~Hz}$ repeated at $0.025 \mathrm{~Hz}$. (A) The application of CTZ (light line) reduced depression, prolonged EPSC decay, and led to a significant residual current, compared to control conditions (solid line; average of five example traces in each condition from one recording, stimulation artefacts were removed). (B) Average cumulative EPSC amplitude. Estimating the pool size as the $y$-axis intercept of a linear fit to the cumulative over the range of stimulus 10 to 21 yielded $13.2 \mathrm{nA}$ (control) and $31.4 \mathrm{nA}$ (CTZ). The average ratio between the pool estimate in the absence and presence of CTZ was $2.4 \pm 0.1$ (mean $\pm S E M$; $n=$ 6). (C) Average ratio of EPSC amplitudes in absence of CTZ over EPSC amplitudes in presence of CTZ, and comparison to the calculated quantal size reduction under control conditions (from Fig. 18). (D) Plot of the pool estimate from the backextrapolated fit to the cumulative EPSC amplitudes versus the cumulative EPSC amplitude at the $5^{\text {th }}$ response. A linear fit yielded an offset of $-1.55 \mathrm{nA}$ and a slope of 1.09 . 
presence of CTZ the pool estimate was on average $2.4 \pm 0.1(n=6)$ times larger than the estimate under control conditions. With respect to the results of the variance and covariance analysis the pool estimates under control conditions (as in Schneggenburger et al. (1999)) must be considered to underestimate the pool size. Furthermore the difference between the pool estimate at $2 \mathrm{mM}$ and $4 \mathrm{mM}\left[\mathrm{Ca}^{2+}\right]_{\mathrm{ext}}$ reported by Schneggenburger et al. (1999) could have arisen only because the initial response at $4 \mathrm{mM}\left[\mathrm{Ca}^{2+}\right]_{\mathrm{ext}}$ is larger than at $2 \mathrm{mM}$ and contributes considerably to the cumulative EPSC amplitudes before significant desensitisation sets in. This is substantiated by the fact, that the difference in the pool size estimate is very similar to the difference in the response size at $2 \mathrm{mM}$ and $4 \mathrm{mM}\left[\mathrm{Ca}^{2+}\right]_{\text {ext }}$.

In order to find out, how much information about the pool can be gained from the cumulative of the EPSCs in response to the standard five stimuli train protocol, the cumulative EPSC amplitude at the $5^{\text {th }}$ stimulus of the 21 stimuli trains was compared to the pool estimate obtained by back-extrapolation (see Fig. 23D). The plot shows that both quantities are almost identical. A straight line fit yields an offset of $1.55 \mathrm{nA}$ and a slope of 1.09. The cumulative EPSC amplitudes of the standard five stimuli experiments are given in Table 4. In case of control conditions and in the presence of CTZ alone they were corrected for the quantal size reduction found in each individual cell. The quantal size estimates obtained from the covariance analysis in case of control conditions and in the presence of CTZ, and from the variance-mean analysis in case of combined application of CTZ and Kyn were utilised in order to express the pool estimates as numbers of vesicles. The average pool estimates were $733 \pm 11(\mathrm{n}=$ 10) vesicles under control conditions, $1432 \pm 8$ (11) vesicles in the presence of CTZ, and $1124 \pm 14$ (9) vesicles under combined application of CTZ and Kyn. Note that the utilized quantal sizes were q' (see eq. M14), and not corrected for the quantal size variability obtained from mEPSC measurements. By this the pool size estimates are comparable to the uncorrected estimates of the numbers of release sites N', for which the true correction factor cannot be determined (see eq. M15).

\subsection{Stationary and non-stationary noise analysis combined}

In order to get an estimate of the reduction in quantal size independent of the covariance analysis, stationary noise analysis and non-stationary noise analysis were combined. First, EPSCs were recorded in response to $0.25 \mathrm{~Hz}$ stimulation at 0.8 or 

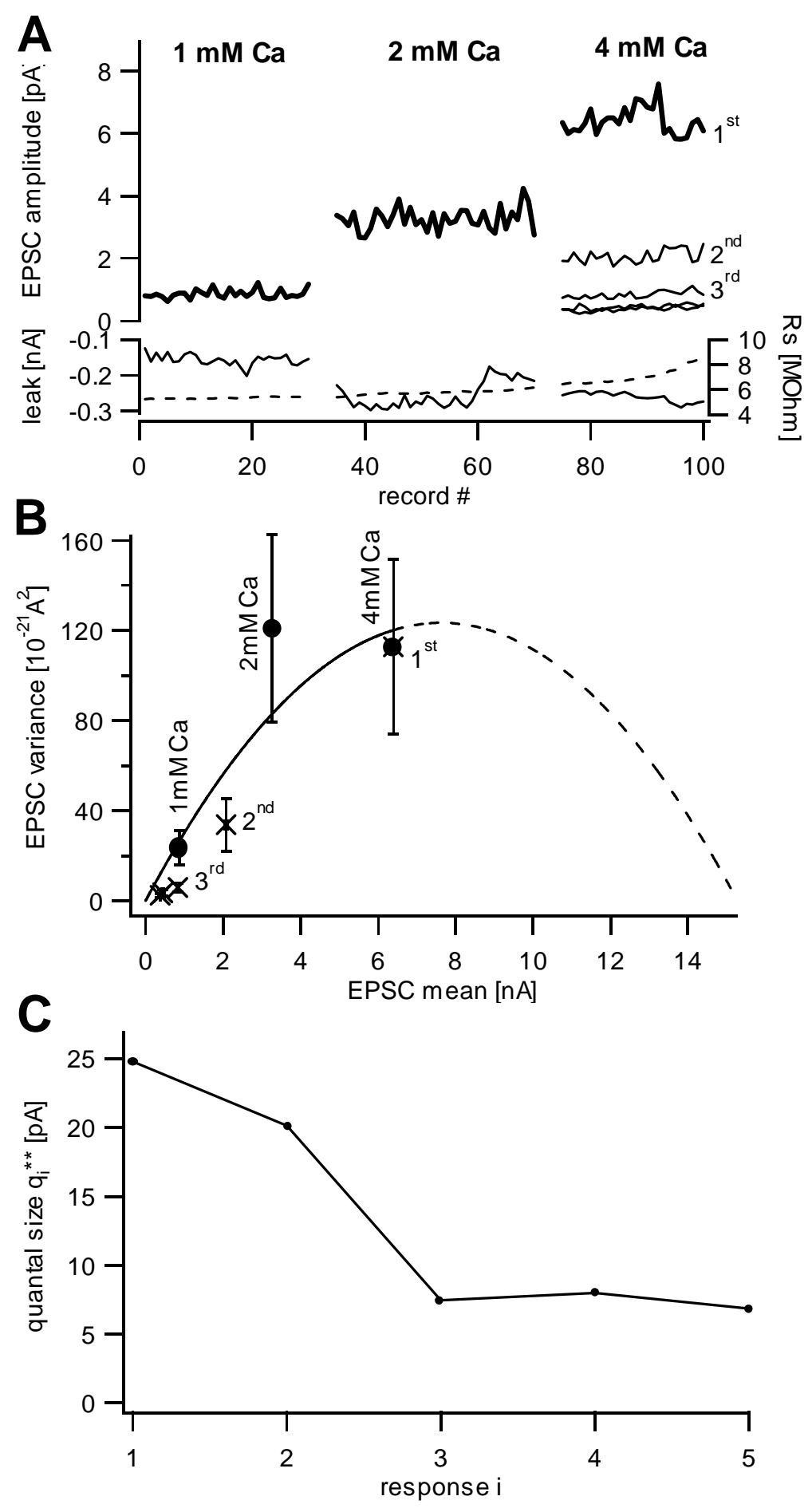

Fig. 24: Experiments combining data from several extracellular calcium concentrations. The standard protocol under control conditions $\left(4 \mathrm{mM}[\mathrm{Ca}]_{\mathrm{ext}}\right)$ was combined with low frequency stimulation at $0.25 \mathrm{~Hz}$ at $1 \mathrm{mM}$ and $2 \mathrm{mM}[\mathrm{Ca}]_{\text {ext }}$. (A) Fluctuation plot of the analysed data segments at 1,2 and $4 \mathrm{mM}[\mathrm{Ca}]_{\text {ext }}$. (B) EPSC amplitude variance versus mean plot for train data (crosses) and data from 2 and 1 $\mathrm{mM}[\mathrm{Ca}]_{\text {ext }}$ (circles): The low calcium data together with the $1^{\text {st }}$ EPSC data from the train can be fitted with a parabola $\left(\mathrm{q}^{*}=32.3 \mathrm{pA} ; \mathrm{N}=473\right)$. It is evident, that the remaining train data points lie below the parabola. (C) Plot of the quantal size $\mathrm{q}_{\mathrm{i}}^{* *}$ as calculated from the train data. The initial quantal size is in reasonable agreement with the value obtained from the parabolic fit in (B). 
$1 \mathrm{mM}\left[\mathrm{Ca}^{2+}\right]_{\text {ext }}$ and at $2 \mathrm{mM}\left[\mathrm{Ca}^{2+}\right]_{\text {ext }}$. Then the standard five stimuli train protocol was applied at $4 \mathrm{mM}\left[\mathrm{Ca}^{2+}\right]_{\text {ext }}$ (see Fig. 24A). The first response in the train can be regarded as an isolated response in the respect that it is not influenced by any preceding response. Therefore the variance and mean data from the low frequency and low $\left[\mathrm{Ca}^{2+}\right]_{\mathrm{ext}}$ data was combined with the data from the first response in the train to fit the parabola as expected from the theory. The data from response 2 to 5 in the train was always located below the parabola in the variance-mean plot (see Fig. 24B). In order to quantify the reduction in quantal size at the end of the train, the initial or resting quantal size was compared to the quantal size at the end of the train. The initial or resting quantal size was estimated in three ways as $\mathrm{q}^{*}$ from the variance over mean ratio of the data at both low $\left[\mathrm{Ca}^{2+}\right]_{\mathrm{ext}}$ and from a parabolic fit to the low $\left[\mathrm{Ca}^{2+}\right]_{\mathrm{ext}}$

A
\begin{tabular}{|c|c|c|c|c|c|}
\hline experiment & $\begin{array}{c}\text { var/ave } \\
(0.8 / 1 \mathrm{mM} \mathrm{Ca}) \\
\mathrm{q}^{*}[\mathrm{pA}]\end{array}$ & $\begin{array}{c}\text { var/ave } \\
(2 \mathrm{mM} \mathrm{Ca}) \\
\mathrm{q}^{*}[\mathrm{pA}]\end{array}$ & \multicolumn{2}{|c|}{$\begin{array}{c}\mathrm{q}^{*}[\mathrm{pA} / \text { ave parabolic fit } \\
\text { train fit } \\
(4 \mathrm{mM} \mathrm{Ca}) \\
\mathrm{q}^{*}[\mathrm{pA}]\end{array}$} \\
\hline c040700_03 & 24.0 & 20.2 & $28.1 \pm 7.0$ & $618 \pm 367$ & 6.3 \\
\hline c060700_01 & 37.4 & 60.2 & $49.0 \pm 11.4$ & $545 \pm 183$ & 10.8 \\
\hline c200600_01 & 36.1 & 30.8 & $41.1 \pm 4.8$ & - & 15.5 \\
\hline c220600_01 & 68.1 & 103.1 & $102 \pm 9.8$ & - & 23.4 \\
\hline mean \pm SEM & $38.6 \pm 7.8$ & 42.7 & $33.9 \pm 9.5$ & $438 \pm 363$ & 7.1 \\
\hline
\end{tabular}

B
\begin{tabular}{|c|c|c|c|}
\hline experiment & ratio1 & ratio2 & ratio3 \\
\hline c040700_03 & 0.26 & 0.31 & 0.22 \\
\hline c060700_01 & 0.29 & 0.18 & 0.22 \\
\hline c060700_02 & 0.43 & 0.50 & 0.38 \\
\hline c200600_01 & 0.34 & 0.23 & 0.23 \\
\hline c220600_01 & 0.26 & 0.17 & 0.21 \\
\hline mean \pm SEM & $0.32 \pm 0.03$ & $0.28 \pm 0.06$ & $0.25 \pm 0.03$ \\
\hline
\end{tabular}

Table 5: Summary of experiments where the standard protocol under control conditions was combined with low frequency stimulation at $0.25 \mathrm{~Hz}$ in $2 \mathrm{mM}$ and 0.8 $/ 1 \mathrm{mM}[\mathrm{Ca}]_{\text {ext }}$ (see also Fig. 24). Quantal size measure $\mathrm{q}^{*}$ calcualted as ratio of EPSC amplitude variance over mean for the recordings at $0.25 \mathrm{~Hz}$ in $2 \mathrm{mM}$ and 0.8 / $1 \mathrm{mM}[\mathrm{Ca}]_{\text {ext }}$ and determined from a fit to the data of EPSC3 to EPSC5 in the variance versus mean plot of the train data at $4 \mathrm{mM}[\mathrm{Ca}]_{\mathrm{ext}}$. Ratio 1 is the ratio of the reduced $\mathrm{q}^{*}$ for the late responses in the train at $4 \mathrm{mM}[\mathrm{Ca}]_{\text {ext }}$ over the $\mathrm{q}^{*}$ at $0.8 / 1$ $\mathrm{mM}[\mathrm{Ca}]_{\text {ext }}$. Ratio 2 is the ratio of the reduced $\mathrm{q}^{*}$ over the $\mathrm{q}^{*}$ at $2 \mathrm{mM}[\mathrm{Ca}]_{\text {ext. }}$ Ratio 3 is the ratio of the reduced $\mathrm{q}^{*}$ over the $\mathrm{q}^{*}$ from a parabolic fit to the variance-mean plot of the data obtained from different $\left[\mathrm{Ca}^{2+}\right]_{\mathrm{ext}}$. 
data combined with the data of the first response in the train. The quantal size at the end of the train was determined from the slope of a straight line through the origin fitted to variance-mean plot for response 3 to 5 of the train. The average estimates of the quantal size from each procedure are given in Table 5. The average of the quantal size at the end of the train was $\mathrm{q}_{\mathrm{end}}{ }^{*}=12.6 \pm 3.1 \mathrm{pA}(\mathrm{n}=5)$. This and the average reduction with respect to the three estimates of initial quantal size of about $30 \%$ was in good agreement with the values obtained by the covariance analysis in other cells (see Fig. 10C and 10D). A direct comparison with the calculated quantal size from the covariance within the same cells was here generally not possible. The covariance could not be determined reliably, because the train data sets in these experiments were comparably small. Only in one case a direct comparison to the quantal size $\mathrm{q}^{* *}$ calculated from the covariance was possible (see Fig. 24C) and this showed good agreement with the values determined in the alternative ways as described above. 


\section{Discussion}

\subsection{The method}

An analytical approach referred to as ensemble noise analysis (Clamann et al. 1989), multiple - probability - fluctuation analysis (Silver et al. 1998) or variance - mean analysis (Reid and Clements 1999) was applied to study short term synaptic plasticity in the calyx of Held synapse. It requires the model independent determination of the mean and the variance of synaptic responses under different conditions. The relationship between the two parameters is then compared to the relationship predicted by the considered statistical model of transmission, which is a parabola for the simple binomial model. This approach has significant advantages over the classical quantal analysis including its extensions (Redman 1990, Walmsley 1993). It is less noise sensitive, it provides more and more reliable information, because it integrates or combines the analysis of different conditions (e.g. release probability settings), and furthermore it incorporates extensions in a more comprehensible way, which account for quantal size variation and heterogeneity in the release probability and lead to distortions of the simple parabolic variance - mean relationship.

In the approach taken here intra- and intersite quantal variance (Frerking and Wilson 1996) was accounted for, but heterogeneity in the release probability was neglected (Silver et al. 1998). A new extension was introduced, which considers the apparent release probability to be composed as the product of the probability for a release site being occupied with a release competent vesicle and the probability for this vesicle to be released upon stimulation as suggested by Zucker (1989) and Quastel (1997). The expressions for the variance mean relationship remain the same with this assumption. This extension accounts for use-dependent changes in the apparent release probability for example by depletion and provides some means to gain more information about the mechanisms underlying short term synaptic plasticity. It provides an interpretation of the covariance between successive responses. On the basis of the covariance an equation was derived, which allows to calculate the quantal size for each individual response in a train. The knowledge of the quantal size for each response in the train provides very useful information in the interpretation of short-term synaptic plasticity. The extended method was tested, by applying it to simulated data. The results showed good agreement with the underlying parameters of the simulation, and any deviations 
could be accounted for in especially considered complications in some simulation scenarios, such as heterogeneity in the release probability.

\subsection{Application of the methods and summary of the results}

So far the variance-mean analysis was applied to steady sequences at various conditions regulating the mean response size (Silver et al. 1998, Reid and Clements 1999, Oleskewich et al. 2000). Oleskewich et al. (2000) also recorded paired-pulse responses at different conditions regulating the mean responses amplitude and applied the analysis for each responses separately. Here instead, in order to analyse synaptic short-term plasticity it was applied to non-stationary responses evoked by short trains of stimuli repeated many times in order to obtain the data for statistical analysis, which is similar to the approach of Clamann et al. (1989).

Preliminary experiments were performed at the frog nmj (Xenopus laevis) in embryonic nerve-muscle co-culture (for the preparation and electrophysiological methods refer to Ashery et al. (1999)). Some results were obtained, which showed agreement with binomial parameters reported for the frog nmj by Wernig (1974). However this preparation proofed to yield results of extensive variability, very likely because of the early developmental stage. Therefore the experiments were continued at the calyx of Held synapse, for which the applicability of the variance-mean analysis had already been shown by Meyer (1999).

This synapse exhibits strong use dependent depression (von Gersdorff et al. 1997) and the aim was to gain more insight into the underlying mechanisms. The presented analysis shows that under the condition of elevated release probability at short interstimulus intervals $(10 \mathrm{~ms})$ synaptic transmission at the calyx of Held synapse does not proceed according to binomial statistics. Depressed responses in a train display less variance in the EPSC amplitude than expected from binomial statistics. This deviation suggests a postsynaptic phenomenon, i.e. a reduction in quantal size, such that previous release causes a reduction in quantal size of subsequent release. Strong candidates are postsynaptic receptor saturation (Diamond and Jahr 1997) and postsynaptic receptor desensitisation (Trussel at al. 1993, Otis et al. 1996a). This apparent reduction in quantal size was quantified with respect to the response number applying the analysis of the covariance between successive stimuli. The final quantal size in a five stimuli train was found to be reduced to about $30 \%$ of the initial or resting value. Application of $100 \mu \mathrm{M} \mathrm{CTZ}$, a drug that prevents AMPA receptor 
desensitisation (Trussel et al. 1993), yielded transmission, which was in concord with binomial statistics as judged from variance versus mean plots. However, analysis of the quantal size based on the covariance method still showed a remaining quantal size reduction of about $25 \%$. This could be attributed to the second mechanisms for quantal size reduction, i.e. receptor saturation. Combined application of CTZ and 1 mM kynurenic acid, a fast AMPA receptor agonist, which prevents receptor saturation (Diamond and Jahr 1997, Liu at al. 1999), abolished the remaining quantal size reduction.

Thus the combined application of CTZ and Kyn renders the postsynaptic part of transmission constant and stationary, such that the remaining depression observed must be attributed to the presynapse. Activation of presynaptic metabotropic glutamate receptors was shown to contribute $<10 \%$ to depression at 5 to $10 \mathrm{~Hz}$ stimulation frequency (von Gersdorff et al. 1997). However in short trains at $100 \mathrm{~Hz}$ the presynaptic calcium current was shown to facilitate slightly (Fig. 2A in Borst and Sakmann 1998). This shows that calcium current inactivation (Forsythe et al. 1998) and activation of metabotropic autoreceptors, which were shown to modulate the presynaptic calcium current (Takahashi et al. 1996), do not contribute to depression in the presented conditions of 5 stimuli at $100 \mathrm{~Hz}$. Therefore it can be argued that vesicle depletion is the reason for the remaining depression observed in absence of postsynaptic receptor saturation and desensitisation.

\subsection{Synchronous and asynchronous release}

The process shaping the EPSC is contentious. Its decay could be dependent on the neurotransmitter sequestration by any process and the resulting inactivation of the postsynaptic receptors, on desensitisation of the postsynaptic receptors, and on the time dispersion of the release events (Trussel et al. 1993, Borst and Sakmann 1996). It is most likely, a superposition of the mentioned mechanisms, and the question here is not to elucidate the different contributions, but rather what errors arise by using the peak EPSC amplitude as a measure for the number of vesicles undergoing fusion in response to a stimulus, and not e.g. the EPSC charge (Bekkers and Stevens 1995).

Borst and Sakmann (1996) showed, that evoked quantal EPSCs at $0.25 \mathrm{mM}\left[\mathrm{Ca}^{2+}\right]_{\mathrm{ext}}$ were dispersed in time. The majority of release events occurred within a time window of 1 to $2 \mathrm{~ms}$ and the latest events happened about $4.5 \mathrm{~ms}$ after the earliest. Quantal EPSCs at $0.25 \mathrm{mM}\left[\mathrm{Ca}^{2+}\right]_{\text {ext }}$ had a faster time course than the multi-quantal EPSCs at 
$2 \mathrm{mM}\left[\mathrm{Ca}^{2+}\right]_{\text {ext }}$. The slower time course of the multi-quantal EPSC could be accounted for by the time dispersion found for quantal-EPSCs. Borst and Sakmann (1996) determined quantal content estimates by dividing the EPSC amplitude by the mEPSC amplitude (43 $\pm 5 \mathrm{pA})$, and by dividing the EPSC charge by the mEPSC charge ( $83 \pm 11 \mathrm{fC}$ ), and obtained $151 \pm 14$ and $210 \pm 22$ vesicles, respectively. Thus the peak amplitude, which is considered to account for synchronous release, underestimates the total quantal content, comprising synchronous and asynchronous release, which is accounted for by the charge measurement (Beckers and Stevens 1995, Borst and Sakmann 1996).

The implications for the analysis presented here are the following. Fig. 22B shows that EPSC peak and EPSC charge are proportional under control conditions. This is in agreement with the suggestion by Borst and Sakmann (1996) that the distribution of quanta released per time scales uniformly in making up a multi-quantal EPSC of a certain size. The proportionality factor relating EPSC amplitude and charge is $0.49 \mathrm{~ms}^{-1}$, as obtained here for control conditions. Expressing it as the ratio of synchronous plus asynchronous release over synchronous release alone yields 1.3, based on the EPSC amplitude and charge data by Borst and Sakmann (1996), and compares well to the ratio of the quantal content estimates they obtained. The prolonged EPSC decay in the presence of CTZ (see Fig. 21A and Fig. 22A), should not be due to asynchronous release, uncovered by the inhibition of desensitisation according to Borst and Sakmann (1996), but rather be the result of the so called residual current arising from build-up of the released glutamate in the synaptic cleft (Trussel et al. 1993, Otis et al. 1996) as already mentioned. Thus if the charge of the EPSC would be used to quantify neurotransmitter release (Bekkers and Steven 1995, Borst and Sakmann 1996), one would have to quantify and to subtract the residual current (see Neher and Sakaba 2000). Therefore it has technical advantages to use the EPSC peak amplitude above background current for analysing the amount of neurotransmitter release, bearing in mind, that it accounts directly only for synchronous release, but that asynchronous release and synchronous are proportional, and the overall release is about 1.3 times the synchronous release.

Regarding the fluctuation analysis of the EPSC amplitudes, the time dispersion of quantal events making up a multi-quantal EPSC introduces a kind of quantal size variation. This is, because the EPSC peak amplitude samples the individual events at 
the time of the peak, which is at some time along their individual time course. The amplitudes of the quantal size time course samples contained in the peak range from zero, in case an event is not captured by the peak at all, to the quantal event peak amplitude, in case of coincidence of individual event peak and multi-quantal EPSC peak. The effect of this on the noise analysis is, that it reduces the quantal size average and at the same time introduces quantal amplitude variability, which increases the apparent quantal size q', similar to intra- and intersite quantal variability

\begin{tabular}{|c|c|c|c|c|}
\hline & $\begin{array}{c}\mathrm{q}^{*} \\
{[\mathrm{pA}]}\end{array}$ & $\begin{array}{c}\mathrm{q}_{1}^{* *} \\
{[\mathrm{pA}]}\end{array}$ & $\begin{array}{c}\text { mEPSC q } \\
{[\mathrm{pA}]}\end{array}$ & $\begin{array}{c}\text { block. / pot. } \\
\text { factor }\end{array}$ \\
\hline contol & - & $46.7 \pm 6.7$ & $40.4 \pm 8.6$ & - \\
\hline CTZ & $31.6 \pm 2.8$ & $44.5 \pm 6.7$ & $46.5 \pm 8.5$ & $1.11 \pm 0.03$ \\
\hline CTZ + Kyn & $22.6 \pm 6.5$ & $22.4 \pm 6.0$ & $(21.0)$ & $0.50 \pm 0.02^{1)}$ \\
\hline
\end{tabular}

Table 6: Comparison of the quantal size measures $\mathrm{q}^{*}$ and $\mathrm{q} * *$ obtained by the different approaches with the q' from directly recorded quantal events (mEPSCs).

1) From Neher and Sakaba (2000) comparing $100 \mu \mathrm{M}$ CTZ alone and $100 \mu \mathrm{M} \mathrm{CTZ}$ plus $1 \mathrm{mM}$ Kyn.

(see eq. M14 amd M22). With respect to the release site number estimate N', the effect depends on whether the quantal amplitude sample variability is more equivalent to intra- or intersite quantal variability (see eq. M15 and M18). Borst and Sakmann (1996) suggest, that the observed time dispersion of individual quantal events making up the multi-quantal EPSC results from the difference in diffusional distance between $\mathrm{Ca}^{2+}$-channels and $\mathrm{Ca}^{2+}$-sensor of the vesicular release machinery. This argues for considering the quantal amplitude sample variability as variability of the intersite type. In that case the dispersion of the amplitudes would have an effect on the estimate of the number of release sites. On the other hand, it can be argued, that due to the stochastic operation of ion channels, the $\mathrm{Ca}^{2+}$-concentration profile varies between stimulations. This would cause variable time dispersion and amplitude sampling variability of the intrasite type, which has no influence on the estimate of the number of release sites N'.

\subsection{Consistency of the results}

Before discussing the observed phenomena with respect to the underlying mechanisms and interpretation in view of other studies, the consistency of the results is demonstrated. The absolute quantal size was determined here in up to three 
different ways: From the variance - mean plot, from the analysis of the covariance, and from mEPSC recordings. In addition the determined quantal sizes for different conditions regarding drug application can be compared on basis of the blocking or potentiation factor determined for evoked responses. There is good agreement between the quantal sizes determined in the different ways under the different conditions as well as regarding the potentiation or blocking factors comparing the data between conditions see Results Table 3. Only the $\mathrm{q}^{*}$ value for the CTZ case deviates considerably from the other data, but this is readily explained on the basis of the finding that under CTZ the covariance analysis reveals a quantal size reduction by about $29 \pm 5 \%(\mathrm{n}=11)$ for the depressed responses in the train (see Fig. 18). The value of $\mathrm{q}^{*}$ from a parabola or straight line fitted to the data in the variance - mean plot reflects this reduced quantal size.

This leads to considering the consistency of the quantal size reduction quantified for each response in the train with the analysis of the covariance (see Fig. 18). This can be assessed by comparing the response averages in the train under control conditions and in presence of CTZ or CTZ and Kyn, which partly or completely prevents the quantal size reduction. Fig. 19C shows the results obtained by dividing the average normalised responses in the train under control conditions and in the presence of CTZ by the average normalised response in the presence of CTZ and Kyn. For control conditions this agreed perfectly with the quantal size reduction as calculated on the basis of the covariance. In contrast for CTZ the agreement is poor, but this might result from the comparison being not within the same cells. The response averages might not be completely representative in case of CTZ and CTZ plus Kyn. Under these conditions the short-term plasticity exhibited by the individual cells ranged from immediate depression to strong transient facilitation.

Within cells, a comparison of control conditions and conditions with partially restored quantal size by CTZ was possible in the pool estimation data set (see Fig. 23). The ratio of the average amplitude from each response under control conditions over those in the presence of CTZ shows to what percentage the amplitudes under control conditions are reduced due to desensitisation (see Fig. 23C). This should compare to the quantal size for each response in the train calculated from the covariance data in control conditions expressed as percentage of initial quantal size (see Fig. 18D). However, the calculated quantal size includes a reduction by desensitisation as well as 
saturation and should therefore be lower than the percentage to which response amplitudes under control conditions are reduced compared to CTZ in the stimulus train. In contrast the contrary was observed (see Fig. 23C). The explanation for this might simply be, that the stimulation conditions were different in the pool estimation experiments compared to the standard train protocol. In pool estimation experiments trains of 21 stimuli at $100 \mathrm{~Hz}$ were given every $40 \mathrm{~s}$ in order to allow for complete recovery whereas in the covariance data set trains of 5 stimuli at $100 \mathrm{~Hz}$ were given every $10 \mathrm{~s}$. In contrast to the long $40 \mathrm{~s}$ inter-train interval, the short inter-train interval of $10 \mathrm{~s}$ led to some decline in the first train amplitude over the initial three repetitions. Therefore the amount of neurotransmitter released by the initial responses in the pool estimation experiments was higher than in the standard train experiments, and this caused most likely more desensitisation such that quantal size was stronger reduced. Nevertheless both curves are qualitatively in good agreement.

One more consistency test was available from an extra set of experiments, where low frequency stimulation at different $\left[\mathrm{Ca}^{2+}\right]_{\mathrm{ext}}(0.8,1,2$ and $4 \mathrm{mM})$ was combined with the five stimulus protocol at $4 \mathrm{mM}\left[\mathrm{Ca}^{2+}\right]_{\mathrm{ext}}$ (see Fig. 24A). The variance and mean from the stationary low frequency stimulation data at different $\left[\mathrm{Ca}^{2+}\right]_{\text {ext }}$ including the first response in the train at $4 \mathrm{mM}\left[\mathrm{Ca}^{2+}\right]_{\mathrm{ext}}$, which could be regarded as a unitary response in the respect that no response was immediately preceding it, gave the parabolic plot as expected from the theory. The data from the remaining responses in the train at $4 \mathrm{mM}\left[\mathrm{Ca}^{2+}\right]_{\text {ext }}$ displayed the deviation from the parabola as already described above. This allowed to quantify the amount of quantal size reduction at the end of the train relative to the resting quantal size. The reduced quantal size at the end of the train was determined from the slope of a line fit to the data from $3^{\text {rd }}$ to $5^{\text {th }}$ response passing through the origin. The resting quantal size was determined both from a parabolic fit to the data from $\left[\mathrm{Ca}^{2+}\right]_{\mathrm{ext}}$ variation and by the ratio of variance over mean for the lowest $\left[\mathrm{Ca}^{2+}\right]_{\mathrm{ext}}$. Because the train data sets in these experiments were comparably small, the covariance could not be determined reliably, so that only in one case a direct comparison to the quantal size $\mathrm{q}^{* *}$ calculated from the covariance was possible and this showed agreement (see Fig. 24C). However the average ratios of reduced quantal size over resting quantal size summarised in Table 5 exhibit good agreement with the quantal size reduction as calculated from the covariance in the data set of Fig. 18C. 


\subsection{Comparison of the reduction in quantal size to other studies}

A reduction in quantal size can have postsynaptic as well as presynaptic reasons. At the postsynapse receptor desensitization is known to contribute to synaptic depression (Trussel et al. 1993) and to reduce the quantal size immediately after an evoked release event (Otis et al. 1996a) in calyx type synapses. Furthermore saturation of postsynaptic receptors can occur during synaptic transmission (Tong and Jahr 1994). Recently a presynaptic effect was reported, which causes a reduction in quantal size in a use dependent manner by incomplete refilling of rapidly recycling vesicles with neurotransmitter (Behrends and Rumpel 2000). However, the reduction in quantal size observed here could be completely explained by the postsynaptic effects, because they were abolished completely by combined application of CTZ and Kyn, which prevent AMPA receptor desensitization (Trussel et al. 1993) and saturation (Diamond and Jahr 1997), respectively. The quantal size reduction under control conditions observed here caused a deviation from the variance-mean parabola very similar to the deviations observed for double pulses in the rat endbulb of Held synapse reported by Oleskevich et al. (2000). They showed directly that after a paired pulse the quantal size is reduced by $20.9 \pm 2.7 \%$ in a comparison of the amplitudes of mEPSC recorded before the paired pulse and within $30 \mathrm{~ms}$ after the second stimulus at $4 \mathrm{mM}\left[\mathrm{Ca}^{2+}\right]_{\text {ext }}$. The reduction observed here is more than twice the reduction reported by Oleskevich et al. (2000). This discrepancy might arise, because during the $30 \mathrm{~ms}$ recording interval some recovery from desensitisation or saturation might have occurred already. A further reason could be technical difficulties in identifying quantal events in the tail of the evoked response, which generally leads to missing the small events and overestimation of the mean. However, the amount of reduction after the first stimulus down to $54.8 \pm 6.9 \%$ observed here is remarkably similar to the quantal size reduction of $>46 \%$ measured directly by recording mEPSCs after an EPSC at $3 \mathrm{mM}$ $\left[\mathrm{Ca}^{2+}\right]_{\text {ext }}$ in a calyx type synapse in the avian nucleus magna cellularis (Otis et al. 1996a). An even more direct comparison is possible in the rat calyx of Held synapse with data obtained by a combination of deconvolution and a variant of non-stationary fluctuation analysis to EPSCs evoked by controlled depolarization of the presynapse in double-patch recordings (Neher and Sakaba 2000). In this study an EPSC with a quantal content of $207 \pm 59$ vesicles was elicited under control conditions, which is comparable to the quantal content of the first response in the train at $4 \mathrm{mM}\left[\mathrm{Ca}^{2+}\right]_{\mathrm{ext}}$ 
here. This response caused a quantal size reduction down to $42 \pm 5 \%$, which was restored to a value of $83 \%$ in the presence of $100 \mu \mathrm{M} \mathrm{CTZ} \mathrm{(following} \mathrm{a} \mathrm{response}$ with a quantal content of about 300 vesicles). Combined application of $100 \mu \mathrm{M} \mathrm{CTZ}$ and $1 \mathrm{mM}$ Kyn completely abolished the apparent quantal size reduction. Here in the presence of $100 \mu \mathrm{M} \mathrm{CTZ}$ the quantal size was reduced to $74.2 \pm 8.9 \%$ after the first response, and the combination with $1 \mathrm{mM}$ Kyn completely removed any quantal size reduction. Thus, the approach by Neher and Sakaba (2000), which is independent from the methods applied here yields very similar results in the same preparation as studied here.

\subsection{Discussion of the covariance}

The equation derived here for the covariance from the binomial model with parameters $\mathrm{N}, \mathrm{q}, \mathrm{p}_{0}, \mathrm{p}_{\mathrm{A}}$ and $\mathrm{p}_{\mathrm{Aj} j \mathrm{i}}$ is equivalent to the expressions published by VereJones (1966) and Quastel (1997). Based on depletion of vesicles available for release from a fixed number of release sites the equations predict negative correlation between the amplitude of successive responses in a train. This was always observed between consecutive EPSCs in the 5 stimuli train, except in a few cases, where there was no correlation observed between the late, depressed responses. In contrast if release is not constraint to a fixed number of release sites, but depends only on the number of vesicle available for release, the occurrence of positive correlation is predicted for certain conditions, too (Vere-Jones 1966, Matveev and Wang 2000). The absolute covariance was maximal in the first pair of responses, and progressively declined with increasing number of stimuli, as predicted by Vere-Jones (1966) and Quastel (1997). Similarly the absolute correlation coefficient was maximal in the first pair of responses, and progressively declined with increasing number of stimuli. However, in contrast to the covariance the absolute correlation coefficient in CTZ was considerably smaller compared to control conditions, and with combined application of CTZ and Kyn it was even further reduced. The implications of this are discussed below.

Reports concerning the evaluation of correlation in recordings of synaptic transmission are rare in the literature. There are two reports at the nmj, one for the correlation between the first and second response and the other for the correlation during the steady-state phase in a train. Elmquist and Quastel (1964) showed negative correlation between first and second response in tetanic stimuli at 40 and $80 \mathrm{~Hz}$ in 2 
$\mathrm{mM}\left[\mathrm{Ca}^{2+}\right]_{\mathrm{ext}}$, with a correlation coefficient of -0.596 . During the steady-state response phase covariance between successive stimuli was found to be not significantly different from zero (Zucker 1973). Both findings are in agreement with the observation at the calyx of Held synapse. For the CNS there are no explicit evaluations of the correlation coefficient available. However a number of studies showed negative correlation. At the synapses of pyramidal cells onto multipolar interneurons in the neocortex, during depressing triple pulses, it was observed, that responses were larger if the preceding response failed to occur, and that the failure rate of the second response increased by $9 \%$ if the first response occurred (Reyes et al. 1998). In the hippocampus monosynaptic contacts of CA3 neurons onto CA3 or CA1 neurons displayed an inverse relationship in the paired-pulse responses. If the first EPSC was smaller than its mean, the second was found to be $4.3 \pm 0.8 \%$ greater than its mean, conversely, if the first EPSC was larger than its mean, the second was $3.7 \pm 0.7 \%$ lower than its mean (Debanne et al. 1996, Thompson et al. 1998). All these reports attributed the negative correlation to depletion. In contrast the connection of pyramidal cells onto bitufted gabanergic interneurons, which is facilitating in response to the triple pulses as mentioned above, did not display correlation (Reyes et al. 1998). Furthermore Mauthner cell axons connecting onto cranial relay interneurons display no correlation in paired pulses (Faber et al. 1998, Waldeck et al. 2000). Kraushaar and Jonas (2000) report absence of correlation between paired pulse responses with $100 \mathrm{~ms}$ inter-stimulus interval and slight correlation between consecutive responses in the late portion of trains at $20 \mathrm{~Hz}$ in 2 $\mathrm{mM}\left[\mathrm{Ca}^{2+}\right]_{\mathrm{ext}}$ at the connection between dentate gyrus basket cells and granule cells in the rat hippocampus.

The theory, on which the analysis of the covariance and the calculation of the quantal size $\mathrm{q}^{* *}$ was based on, considered vesicle depletion as the only source of correlation between successive EPSCs. However, the application of this theory showed the presence of postsynaptic receptor desensitisation and postsynaptic receptor saturation. Both of these processes are likely to give rise to negative correlation, too, which is not considered in the theory. From the plot of the correlation coefficient versus stimulus number (see Fig. 18B), it is evident, that desensitisation and saturation contribute to correlation between successive responses. For the first pair of responses in the train the correlation coefficient was $-0.44 \pm 0.05(n=10)$ under control conditions, in the 
presence of CTZ alone it was $-0.17 \pm 0.05(\mathrm{n}=11)$, and in the presence of both CTZ and Kyn it was hardly significantly different from zero.

This suggests, that the presented theory proofs itself not to be valid. However, it was possible to obtain expressions analogous to the equations presented in the methods part. These show, that the original approach is still valid under certain conditions, when the assumption of the independence of release sites is suspended partially (see appendix E). The calculations in appendix E allow, that previous release events at any site have an influence on the quantal amplitude of subsequent release events at the same and any other site. By this the quantal size at subsequent responses is correlated with the amount of release at a previous response. The outcome of the calculation are additional factors in the expression for the covariance between consecutive responses (compare eq. M11 and eq. E12). The additional factor (in the following referred to as $\mathrm{D}_{\mathrm{ij}}$ for desensitisation) to the covariance in the case of negligible refilling (compare eq. M16 and eq. E13) contains the correlation term for the correlation described above relative to the mean of the previous amount released and the subsequent quantal size. It is suggestive that this relation is constant and independent of the stimulus number under certain conditions. In that case the complete additional factor $\mathrm{D}_{\mathrm{ij}}$ would be constant, and if it is known, the original approach can be applied as before with

$$
\mathrm{q}_{\mathrm{i}}=\frac{\operatorname{Var}_{\mathrm{i}}}{\overline{\mathrm{Y}}_{\mathrm{i}}}-\mathrm{D} \cdot \frac{\operatorname{Cov}_{\mathrm{i}, \mathrm{i}+1}}{\overline{\mathrm{Y}}_{\mathrm{i}, \mathrm{i}+1}}
$$

Whether $\mathrm{D}_{\mathrm{ij}}$ is constant or not can be tested by computing the ratio of the covariance between consecutive responses over the product of their means (eq. E14) along the stimulus train. If this ratio, which is equivalent to (eq. M17) is constant, then $D_{i j}=D$ is constant. If the number of release sites $\mathrm{N}$ is known, then $\mathrm{Dij}$ can even be determined by the above mentioned ratio. However, this is not neccessary, if mEPSC data is available. In case $D_{i j}$ is constant along the stimulus train, it can be determined such that calculated initial quantal size on basis of the covariance equals the average quantal size of the mEPSC data (Note that quantal size variability has been neglected here). This would be in some way a calibration of the quantal size estimation to the mEPSC mean as reference value. 
In the data presented here the ratio of the product of the means over the covariance of successive EPSCs (eq. M17), in Table 3 given as parameter $\mathrm{N}_{1}{ }^{* *}$, was found to be constant along the stimulus train, suggesting that $\mathrm{D}_{\mathrm{ij}}$ is constant. Only for the late

A
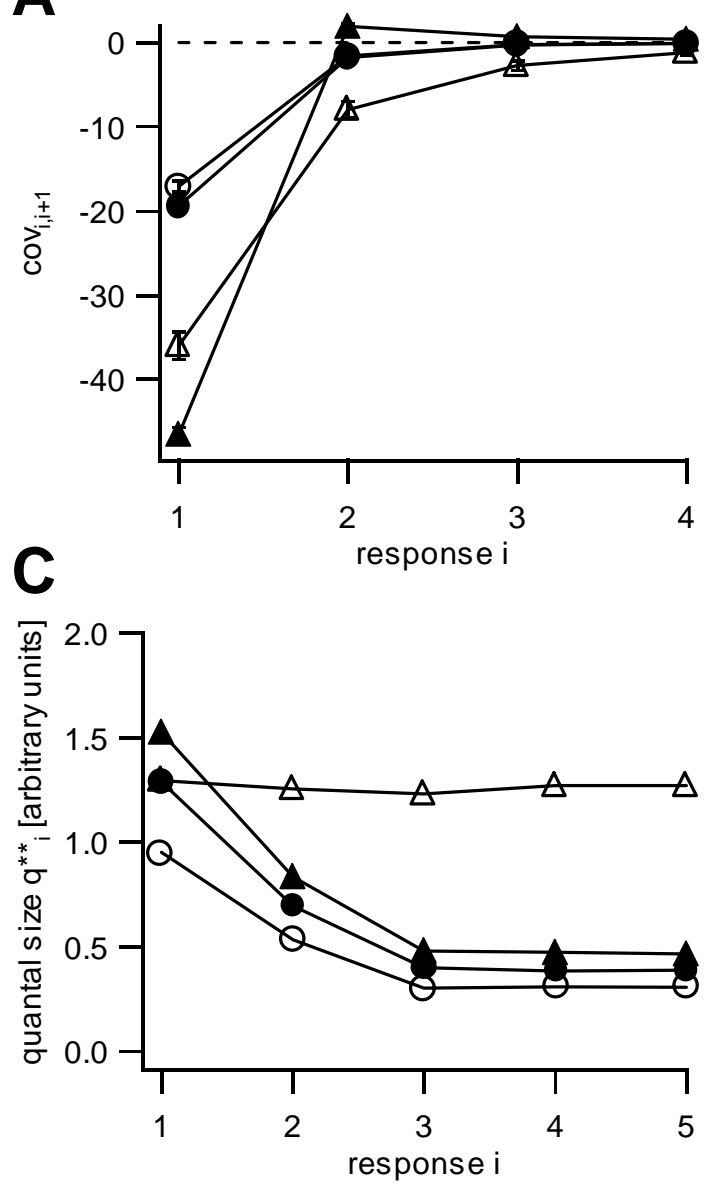

B

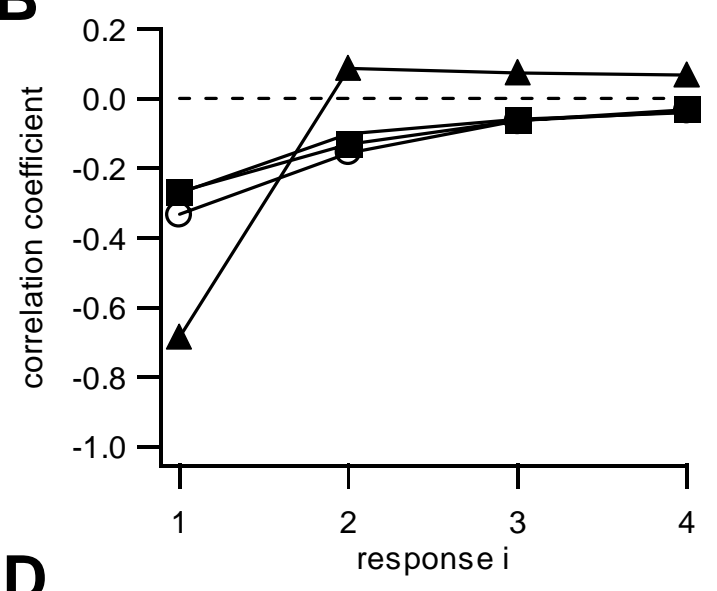

D

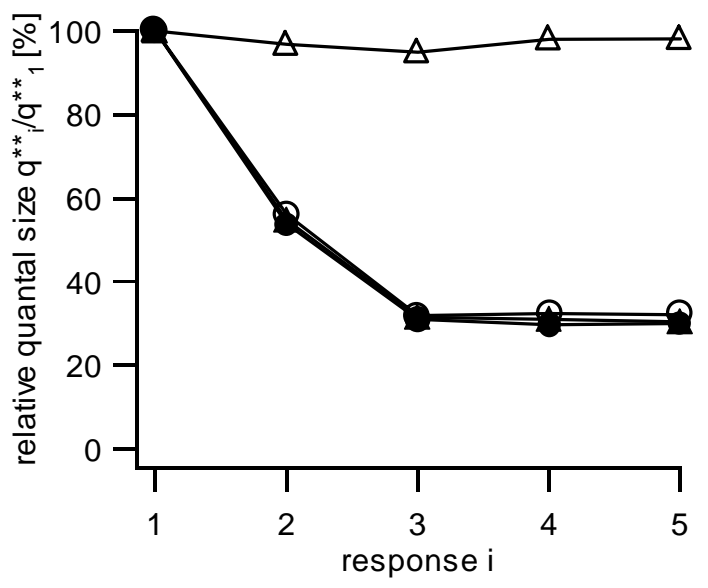

Fig. 25: Preliminary results of simulations including a quantal size reduction. The simulations were performed with $\mathrm{N}=500$ release sites, uniform and constant release probability of 0.5 and an initial site occupancy of $\mathrm{p}_{\mathrm{A}}=0.8$, trains of 5 stimuli $10 \mathrm{~ms}$ apart and repeated every $10 \mathrm{~s}$. Four situations were simulated: The absence of any quantal variability and the quantal size reduction was realised by scaling down the responses uniformly by a factor assigned to each stimulus number in the train (open circles); intrasite quantal variability and the same realisation of the quantal size reduction as above (closed circles); intrasite quantal variability, and variable reduction in quantal size according to the amount of release in response to the previous stimulus, such that the average quantal size reduction remains the same as in the cases above (filled triangles); and intrasite quantal variability without any reduction in quantal size as control (open triangles). (A) Plot of covariance between pairs of successive responses versus response number. (B) Plot of the correlation coefficient for pairs of successive responses versus response number. (C) Plot of the calculated quantal size $\mathrm{q}^{* *}$ on basis of the covariance versus response number. (D) Plot of the calculated quantal size $\mathrm{q}^{* *}$ on basis of the covariance normalised to its initial value versus response number. 
depressed responses in the train sometimes deviations were observed, but the absolute covariance between those was so low (see Fig. 18A), that their contribution to the above equation is negligible.

Here the quantal size as calculated on basis of the covariance was only by chance directly equal to the quantal size obtained from mEPSC data under all three conditions applied, which suggests $D=1$. Note that this is no contradiction, in the sense that the value of $\mathrm{D}$ should reflect the presence or absence of correlation due to desensitisation or saturation, because the correlation is contained relative to the average amount of release and the quantal size (see eq. E13), as mentioned above.

On basis of these new approach considering correlation due to desensitisation the discrepancy in the data obtained for $\mathrm{N}^{*}$ and $\mathrm{N}^{* *}$ (see Table 3) could be explained. From eq. M17 and eq. E15 follows $\mathrm{N}^{* *}=\mathrm{ND}$. Assuming, that the true number of release sites the same under all three conditions (control, CTZ alone, CTZ and Kyn) and closely approximated by the estimate $\mathrm{N}^{*}$ from the variance-mean analysis, one would obtain for control conditions $\mathrm{D}=1$, and for CTZ alone and in combination with Kyn $\mathrm{D} \approx 0.4$. However, this is in contradiction to $\mathrm{D}=1$ for all three conditions as suggested above. This contradiction might be solved, if quantal size variation, which is not taken into account in the calculations in appendix E, would be considered.

Preliminary simulations with intrasite quantal variation and correlation of the quantal size reduction along the train with the amount of previous release show, that the relative quantal size changes along the train are well described by the original approach, but that the absolute quantal size is overestimated. This observation is in agreement with the predictions of the extended approach considering correlation between the quantal size and the amount of previous neurotransmitter released, e.g. due to desensitisation, as discussed above.

\subsection{Interpretation of the binomial parameters}

The number of release sites described by the parameter $\mathrm{N}$ in the model is nowadays associated with the number of active zones (Korn et al. 1982, Korn et al. 1994). N is determined from fitting a parabola to variance - mean plots, which was possible here in experiments with CTZ application alone and in combination with Kyn, but not in control experiments. It should be noted, that for a proper determination of $\mathrm{N}$ from the variance-mean plot, data in the high release probability range is required $(\mathrm{p}>0.5$, Clements and Silver 2000), which was not available here. This has to be considered 
when assessing the presented numbers for the parameter $\mathrm{N}$. The average number of release sites was found to be in the range of 399 to 1556 averaging to $\mathrm{N}=896 \pm 241$ (mean $\pm \mathrm{SEM} ; \mathrm{n}=4$ ) in the presence of $\mathrm{CTZ}$ and in the range of 289 to 1129 averaging to $\mathrm{N}=728 \pm 191$ (mean $\pm \mathrm{SEM} ; \mathrm{n}=4$ ) under combined application of CTZ and Kyn. There was no difference expected between both conditions with respect to $\mathrm{N}$ and both values were in agreement. There is no appropriate morphological information about the rat calyx of Held published so far. Preliminary data indicate that the number of morphological active zones is 500 at the calyx of Held. A rather crude estimate can be made from the data published by Casey and Feldman (1988). Per profile in $3 \mu \mathrm{m}$ thick slices they report an average of $15.1 \pm 0.64$ "terminals". Assuming a calyx diameter of $30 \mu \mathrm{m}$ this gives 450 active zones. Schneggenburger et al. (1999) showed that at saturating $\mathrm{Ca}^{2+}$-influx the number of vesicles, which can be released by a single stimulus is about 700 . This number of maximum quantal content is very similar to the values of $\mathrm{N}$ found here.

However data on morphology and binomial $\mathrm{N}$ from the variance - mean analysis is available from the rat endbulb of Held synapse, which shares both structural and functional properties with the calyx of Held synapse. Oleskevich et al. (2000) found values for $\mathrm{N}$ in the range of 26 to 547 averaging to $\mathrm{N}=124 \pm 49(\mathrm{n}=10)$, which they stated, was in agreement with their unpublished morphological data. However Rygo et al. (1996) found that in two different populations of cat endbulbs of Held the number of synaptic specialisations is $1,720 \pm 395$ and $407 \pm 139$, respectively, distinguished by the spontaneous spiking activity of their afferent fibres as assessed by intracellular recordings. Thus agreement with the data presented here can only be assumed to some extend.

As already discussed above good agreement was found between the quantal size $\mathrm{q}^{*}$ and $\mathrm{q}^{* *}$ as determined from the variance-mean plot and from the covariance, respectively, with q' determined from mEPSC distributions. For the mEPSC distributions under control conditions the average $\mathrm{CV}_{\mathrm{q}}$ was $0.50 \pm 0.10(\mathrm{n}=4)$ and in the presence of CTZ it was $\mathrm{CV}_{\mathrm{q}}=0.55 \pm 0.08(\mathrm{n}=4)$. These values are in perfect agreement with the $\mathrm{CV}_{\mathrm{q}}$ of around 0.5, which Borst and Sakmann (1996) obtained from the amplitude distribution of quantal EPSCs at low $\left[\mathrm{Ca}^{2+}\right]_{\text {ext }}$. The relative contributions of intra- and intersite quantal variation could not be assessed (Frerking and Wilson 1996), although this would have been of interest in order to obtain a better 
estimate for $\mathrm{N}$ (see eq. M15). However, the worst case estimate assuming the quantal variation apparent in the recorded mEPSC distributions was completely due to intrasite quantal variability provides an upper limit for the true number of release sites of 1.3 times the estimates for N'. Electrotonic effects as reason for intrasite quantal variation could be excluded at the calyx of Held, and this might be one argument for Olsekevich et al. (2000) to attribute the complete quantal variability found in mEPSC distributions to intrasite quantal variance at the rat endbulb of Held. In contrast Silver et al. (1998) estimated the intrasite quantal variance from the variance at the high release probability end of the variance-mean plot and reported it to be low compared to intersite quantal variance. However such data was not available here and because of structural difference this finding might not apply here. Furthermore this is in contrast to data by Liu and Tsien (1995), who report considerable intrasite variability.

In the variance - mean plots, there was no evidence for heterogeneity in the release probability. However non-uniform release probability was reported for the rat calyx of Held synapse (Wu and Borst 1999, Sakaba and Neher 2000). The reason, that no heterogeneity was observed is probably because only the low release probability half of the parabola in the variance - mean plot was sampled, but distortions of the parabola due to heterogeneity in the release probability appear only in the high release probability part (Silver et al. 1998), unless the heterogeneity in the release probability increases with decreasing mean.

\subsection{General interpretation and outlook}

The results of the statistical analysis of synaptic transmission at the calyx of Held synapse presented here show, that postsynaptic receptor desensitisation and saturation contribute significantly to synaptic depression under conditions of elevated release probability at short inter-stimulus intervals. This might also hold true at physiological release probabilities. The physiological function of desensitisation was suggested to be a compensatory mechanism for the lack of rapid removal of glutamate from the synaptic cleft and could prevent the interaction of transmitter quanta within the synaptic cleft (Trussel et al. 1993).

There are two paradox apparent: One is that the response at the $5^{\text {th }}$ stimulus is only reduced to $50 \%$ relative to the first. The standard five stimulus at $100 \mathrm{~Hz}$ protocol is applied at $4 \mathrm{mM}\left[\mathrm{Ca}^{2+}\right]_{\mathrm{ext}}$, which should give a situation with a release probability of 0.5 at the first stimulus according to the dose response curve in Schneggenburger et 
al. (1999) and negligible recovery in-between stimuli (von Gersdorff et al. 1997). According to this the $5^{\text {th }}$ response should be reduced to 0.5 to the power of five, which equals 0.03. The other paradox is, that although the number of release sites is in the range of 500 to 1000 as determined from the variance-mean analysis, and responses to unitary physiological stimuli never yield higher quantal content, the number of vesicles, which can be released by strong stimuli is much higher. Complete depletion of the release competent vesicles within less than $200 \mathrm{~ms}$ by depolarisation of the presynapse yields 800 to 4400 vesicles in different cells with an average of $2246 \pm$ 303 (Sakaba and Neher 2000) and an average about 2000 by uncaging of $\mathrm{Ca}^{2+}$ in the terminal (Schneggenburger and Neher 2000).

A solution for the first paradox could be, that replenishment of fusion competent vesicles is faster (Wu and Borst 1999, Sakaba and Neher 2000) than the assumed $4 \mathrm{~s}$ (von Gersdorff et al. 1997). Enhancement of the replenishment by accumulation of intracellular $\mathrm{Ca}^{2+}$ due to high-frequency stimulation (Wang and Kacsmarek 1998) however, has been shown not to be present at the rat calyx of Held synapse (Weis et al. 1999). The reasons for the second paradox could be that single APs cannot cause release of the complete fusion competent pool of vesicles, for example because of heterogeneous release probability due to different $\mathrm{Ca}^{2+}$-sensitivity $(\mathrm{Wu}$ and Borst 1999, Sakaba and Neher 2000) or that asynchronous release is not taken care of in the variance - mean analysis (Borst and Sakmann 1996), such that the number of release sites is underestimated.

However, another explanation is obtained by an alternative interpretation of the apparent release probability to be the product of the release probability per individual release competent vesicle $\mathrm{p}_{0}$ and the probability $\mathrm{p}_{\mathrm{A}}$ that release site is occupied by a release competent vesicle as introduced above (Zucker 1989, Quastel 1997). Instead of a release site being occupied with a single vesicle with the probability $\mathrm{p}_{\mathrm{A}}$ this alternative considers a number of $n$ vesicles docked to each release site (Oleskevich et al. 2000, Waldeck et al. 2000). This is based on the identification of the number of release sites determined by quantal analysis with the number of morphologically defined active zones (Korn et al. 1982, Oleskevich 2000), the observation that active zones have more than one vesicle docked to them (in the hippocampus on average $10.3 \pm 5.6$; Schikorski and Stevens 1997), and finally on the one-vesicle hypothesis (Korn et al. 1994) and lateral inhibition (Dobrunz et al. 1997), which means that upon 
a stimulus a release site can release only one vesicle, because of some steric inhibition of further fusion events.

By this the paradox are explained as follow: In case of an apparent release probability of 0.5 , on average half the sites release a vesicle, but they are not depleted and the remaining vesicles are available for subsequent release upon stimulation. A strong stimulus could release all fusion competent vesicles irrespective of lateral inhibition and yield a vesicle number as a multiple of the number of release sites. This model can further explain readily the correlation found between the determined distribution of active zone sizes (Schikorski and Stevens 1997) and the heterogeneity of the release probability in the hippocampus (Rosenmund et al. 1993, Murthy et al. 1997) on basis of a higher number of vesicles available at larger sites. However heterogeniety proposed on basis of different $\mathrm{Ca}^{2+}$ sensitivity ( $\mathrm{Wu}$ and Borst 1999, Sakaba and Neher 2000) would require the introduction of different release probabilities per individual vesicles.

Quastel (1997) proposed that analysis of the covariance between evoked responses might yield information about whether any change in the apparent release probability reflects changes in $\mathrm{p}_{0}$ or $\mathrm{p}_{\mathrm{A}}$. And this might hold true also for identifying the appropriate model, i.e. single-vesicle sites with occupancy $\mathrm{p}_{\mathrm{A}}$ (Zucker 1989, Quastel 1997) or multi-vesicle sites with $\mathrm{n}$ vesicles (Oleskevich et al. 2000, Waldeck et al. 2000). However it seems to be very complicated, because it would require to find reliably significant differences in the covariance between successive stimuli at various inter-stimulus intervals, i.e. in presence of various degrees of recovery and furthermore information about the latter (see eq. M11). It should be noted that in the theoretical discussion of the multi-vesicle site model Oleskevich et al. (2000) confuse the probability of a release event at a site and the probability for release of each individual vesicle at the site by using the parameter $\mathrm{P}_{\mathrm{r}}$ interchangeably. 


\section{Appendix}

\section{A. Nonstationary-noise analysis in the presence of intra- and intersite quantal variability}

The derivation of the equations considering intra- and inter-site quantal variation are based on the same assumptions as the equations presented in the methods part, except for allowing intra- and intersite quantal variability:

The postsynaptic response to the $\mathrm{i}^{\text {th }}$ stimulus in a train of stimuli at a single release site is given by

$$
\mathrm{y}_{\mathrm{i}}=\mathrm{q}_{\mathrm{i}} \mathrm{x}_{\mathrm{i}} \quad \text { where } \quad \mathrm{x}_{\mathrm{i}}=\left\{\begin{array}{cc}
1 & \text { if vesicle fuses at stimulus } \mathrm{i} \\
0 & \text { otherwise }
\end{array}\right.
$$

with the compound probability for a release event $\mathrm{p}\left(\mathrm{x}_{\mathrm{i}}=1\right)=\mathrm{p}_{\mathrm{Oi}} \mathrm{p}_{\mathrm{Ai}}$

Thus in case a stimulus train is repeated under constant conditions the average amplitude of the postynaptic response to the $i^{\text {th }}$ stimulus at a single release site is

$$
\bar{y}_{i}=<q_{i}>p_{O i} p_{A i}
$$

where $\left\langle\mathrm{q}_{\mathrm{i}}\right\rangle$ is the mean amplitude of quantal currents at the $\mathrm{n}^{\text {th }}$ site due to intrasite quantal variance present in the CNS (Frerking and Wilson 1996, Forti et al 1997). The variance or autocovariance of the postsynaptic response to the $i^{\text {th }}$ stimulus at a single release site is

$$
\begin{aligned}
& \operatorname{var}_{\mathrm{i}}=\operatorname{cov}_{\mathrm{i}, \mathrm{i}}=<\mathrm{y}_{\mathrm{i}}^{2}>-<\mathrm{y}_{\mathrm{i}}>^{2}=<\mathrm{q}_{\mathrm{i}}^{2} \mathrm{x}_{\mathrm{i}}^{2}>-<\mathrm{q}_{\mathrm{i}} \mathrm{x}_{\mathrm{i}}>^{2} \\
& =<\mathrm{q}_{\mathrm{i}}^{2}>\mathrm{p}\left(\mathrm{x}_{\mathrm{i}}=1\right)-<\mathrm{q}_{\mathrm{i}}>^{2} \mathrm{p}\left(\mathrm{x}_{\mathrm{i}}=1\right)^{2} \\
& =\mathrm{p}_{\mathrm{O} i} \mathrm{p}_{\mathrm{Ai}}\left(<\mathrm{q}_{\mathrm{i}}^{2}>-\mathrm{p}_{\mathrm{O}} \mathrm{p}_{\mathrm{Ai}}<\mathrm{q}_{\mathrm{i}}>^{2}\right)
\end{aligned}
$$

where $\left\langle\mathrm{q}_{\mathrm{i}}^{2}\right\rangle$ is the mean squared amplitude of quantal currents at a single site, which can be expressed in terms of mean $\left\langle\mathrm{q}_{\mathrm{i}}\right\rangle$ and variance $\sigma^{2}{ }_{\text {qntra }}$ of the intrasite quantal variation by

Such that

$$
\sigma_{\text {qIntra }}^{2}=<q^{2}>-<q>^{2}
$$

$$
<\mathrm{q}^{2}>=<\mathrm{q}>^{2}+\sigma_{\text {qIntra }}^{2}=<\mathrm{q}>^{2}\left(1+\mathrm{CV}_{\text {qIntra }}^{2}\right)
$$

Under the assumption that the quantal size variance is independent of the stimulus number, combination of eq. A4 and eq. A5b yields for the variance of the postsynaptic response to the $\mathrm{i}^{\text {th }}$ stimulus at a single release site 


$$
\operatorname{var}_{\mathrm{i}}=\mathrm{p}_{\mathrm{Oi}} \mathrm{p}_{\mathrm{Ai}}\left(1+C \mathrm{~V}_{\mathrm{q} \text { Intra }}^{2}-\mathrm{p}_{\mathrm{Oi}} \mathrm{p}_{\mathrm{Ai}}\right)<\mathrm{q}_{\mathrm{i}}>^{2}
$$

The covariance between the amplitude of consecutive responses $i$ and $j$ in the train at a single site is

$$
\begin{aligned}
& \operatorname{cov}_{\mathrm{i}, \mathrm{j}}=<\mathrm{y}_{\mathrm{i}} \mathrm{y}_{\mathrm{j}}>-\bar{y}_{\mathrm{i}} \overline{\mathrm{y}}_{\mathrm{j}} \\
& =<\mathrm{q}_{\mathrm{i}} \mathrm{x}_{\mathrm{i}} \mathrm{q}_{\mathrm{j}} \mathrm{x}_{\mathrm{j}}>-<\mathrm{q}_{\mathrm{i}}><\mathrm{q}_{\mathrm{j}}>\mathrm{p}_{\mathrm{Oi}} \mathrm{p}_{\mathrm{Ai}} \mathrm{p}_{\mathrm{Oj}} \mathrm{p}_{\mathrm{Aj}} \\
& =<\mathrm{q}_{\mathrm{i}} \mathrm{q}_{\mathrm{j}}><\mathrm{x}_{\mathrm{i}} \mathrm{x}_{\mathrm{j}}>-<\mathrm{q}_{\mathrm{i}}><\mathrm{q}_{\mathrm{j}}>\mathrm{p}_{\mathrm{Oi}} \mathrm{p}_{\mathrm{Ai}} \mathrm{p}_{\mathrm{Oj}} \mathrm{p}_{\mathrm{Aj}} \\
& \qquad \begin{aligned}
\text { with } \quad<\mathrm{x}_{\mathrm{i}} \mathrm{x}_{\mathrm{j}}>=\mathrm{p}\left(\mathrm{x}_{\mathrm{j}}=1, \mathrm{x}_{\mathrm{i}}=1\right) \\
=\mathrm{p}\left(\mathrm{x}_{\mathrm{j}}=1 \mid \mathrm{x}_{\mathrm{i}}=1\right) \mathrm{p}\left(\mathrm{x}_{\mathrm{i}}=1\right) \\
=\mathrm{p}_{\mathrm{O} \mathrm{j}} \mathrm{p}\left(\mathrm{z}_{\mathrm{j}}=1 \mid \mathrm{x}_{\mathrm{i}}=1\right) \mathrm{p}_{\mathrm{Oi}} \mathrm{p}_{\mathrm{Ai}}
\end{aligned}
\end{aligned}
$$

where

$$
\mathrm{z}_{\mathrm{i}}= \begin{cases}1 \text { if } \text { site is occupied before stimulus } \mathrm{i} \\ 0 & \text { otherwise }\end{cases}
$$

$$
\begin{array}{ll}
\text { with } & \mathrm{p}\left(\mathrm{z}_{\mathrm{i}}=1\right)=\mathrm{p}_{\mathrm{Ai}} \\
& \mathrm{p}\left(\mathrm{z}_{\mathrm{j}}=1 \mid \mathrm{x}_{\mathrm{i}}=1\right)=\mathrm{p}_{\mathrm{Ajji}}
\end{array}
$$

where $\mathrm{p}_{\mathrm{Aj} \mathrm{i}}$ is the conditional probability that a site is occupied at stimulus $\mathrm{j}$ given it was released at stimulus i. Therefore

$$
\begin{aligned}
\operatorname{cov}_{\mathrm{i}, \mathrm{j}} & =<\mathrm{q}_{\mathrm{i}} \mathrm{q}_{\mathrm{j}}>\mathrm{p}_{\mathrm{Oi}} \mathrm{p}_{\mathrm{Ai}} \mathrm{p}_{\mathrm{O} \mathrm{j}} \mathrm{p}_{\mathrm{Aji}}-<\mathrm{q}_{\mathrm{i}}><\mathrm{q}_{\mathrm{j}}>\mathrm{p}_{\mathrm{Oi}} \mathrm{p}_{\mathrm{Ai}} \mathrm{p}_{\mathrm{O} \mathrm{j}} \mathrm{p}_{\mathrm{Aj}} \\
& =\mathrm{p}_{\mathrm{O} \mathrm{i}} \mathrm{p}_{\mathrm{Ai}} \mathrm{p}_{\mathrm{Oj}}\left(<\mathrm{q}_{\mathrm{i}} \mathrm{q}_{\mathrm{j}}>\mathrm{p}_{\mathrm{Aji}}-<\mathrm{q}_{\mathrm{i}}><\mathrm{q}_{\mathrm{j}}>\mathrm{p}_{\mathrm{Aj}}\right)
\end{aligned}
$$

It is very unlikely that there is a correlation between the size among quanta released from the same site; i.e. $\left\langle\mathrm{q}_{\mathrm{i}} \mathrm{q}_{\mathrm{j}}\right\rangle=\left\langle\mathrm{q}_{\mathrm{i}}\right\rangle\left\langle\mathrm{q}_{\mathrm{j}}\right\rangle$. The covariance between the amplitude of consecutive responses $\mathrm{i}$ and $\mathrm{j}$ in the train at a single site is therefore

$$
\operatorname{cov}_{\mathrm{i}, \mathrm{j}}=\mathrm{p}_{\mathrm{Oi}} \mathrm{p}_{\mathrm{Ai}} \mathrm{p}_{\mathrm{Oj}}\left(\mathrm{p}_{\mathrm{Aj \textrm {j }}}-\mathrm{p}_{\mathrm{Aj}}\right)<\mathrm{q}_{\mathrm{i}}><\mathrm{q}_{\mathrm{j}}>
$$

For the general description of the statistical properties of stimulus-evoked release the properties of a single release site as derived above are now expanded to a population of $\mathrm{N}$ release sites. Under the assumptions that release sites are statistically 
independent and quanta released add linearly the statistics of the population is given as the sum of the statistics of the individual sites. In addition the absence of heterogeneity in the release probability is assumed, i.e. all sites have the same release probability $\mathrm{p}_{0}$ and the same average occupancy probability $\mathrm{p}_{\mathrm{A}}$. However we allow sites to have different mean quantal size $\langle q\rangle$ to take into account intersite quantal variance, but assume a uniform quantal size variation $\sigma_{\mathrm{q} I n t r a}$ within sites (Frerking and Wilson 1996).

The average overall response amplitude at the $\mathrm{i}^{\text {th }}$ stimulus is then the sum of eq. A3 over all sites

$$
\bar{Y}_{\mathrm{i}}=\sum_{\mathrm{n}=1}^{\mathrm{N}} \overline{\mathrm{y}}_{\mathrm{n}, \mathrm{i}}=\sum_{\mathrm{n}=1}^{\mathrm{N}}<\mathrm{q}_{\mathrm{n}, \mathrm{i}}>_{\text {Intra }} \mathrm{p}_{\mathrm{Oi}} \mathrm{p}_{\mathrm{Ai}}=\mathrm{p}_{\mathrm{Oi}} \mathrm{p}_{\mathrm{Ai}} \sum_{\mathrm{n}=1}^{\mathrm{N}}<\mathrm{q}_{\mathrm{n}, 1}>_{\text {Intra }}
$$

With the intersite mean quantal size defined as

$$
<\mathrm{q}_{\mathrm{i}}>_{\text {Inter }} \equiv \frac{1}{\mathrm{~N}} \sum_{\mathrm{n}=1}^{\mathrm{N}}<\mathrm{q}_{\mathrm{n}, \mathrm{i}}>_{\text {Intra }}
$$

eq. A11 simplifies to

$$
\overline{\mathrm{Y}}_{\mathrm{i}}=\mathrm{N}<\mathrm{q}_{\mathrm{i}}>_{\text {Inter }} \mathrm{p}_{\mathrm{Oi}} \mathrm{p}_{\mathrm{Ai}}
$$

The variance of the overall response amplitude is given by the sum of eq. A6 over all sites

$$
\operatorname{Var}_{\mathrm{i}}=\sum_{\mathrm{n}=1}^{\mathrm{N}} \mathrm{p}_{\mathrm{Oi}} \mathrm{p}_{\mathrm{Ai}}<\mathrm{q}_{\mathrm{n}, \mathrm{i}}>^{2}\left(1+\mathrm{CV}_{\mathrm{qIntra}}^{2}\right)-\sum_{\mathrm{n}=1}^{\mathrm{N}} \mathrm{p}_{\mathrm{Oi}}^{2} \mathrm{p}_{\mathrm{Ai}}^{2}<\mathrm{q}_{\mathrm{n}, \mathrm{i}}>^{2}
$$

According to the assumptions of the absence of heterogeneity in $\mathrm{p}_{0}$, uniform average occupancy $\mathrm{p}_{\mathrm{A}}$ and no difference in intrasite quantal variation between release sites this can be simplified to

$$
\operatorname{Var}_{\mathrm{i}}=\mathrm{p}_{\mathrm{Oi}} \mathrm{p}_{\mathrm{Ai}}\left[1+\mathrm{CV}_{\mathrm{q} \text { Intra }}^{2}-\mathrm{p}_{\mathrm{Oi}} \mathrm{p}_{\mathrm{Ai}}\right] \sum_{\mathrm{n}=1}^{\mathrm{N}}<\mathrm{q}_{\mathrm{n}, \mathrm{i}}>^{2}
$$

The remaining summation over all sites above considers the squared mean quantal amplitude from each release site. Thus it is of the form analogous to eq. A5a but for intersite quantal variance instead of intrasite quantal variance

$$
\sigma_{\text {Inter }}^{2}=\frac{1}{\mathrm{~N}} \sum_{\mathrm{n}=1}^{\mathrm{N}}<\mathrm{q}_{\mathrm{n}}>_{\text {Intra }}^{2}-<\mathrm{q}>_{\text {Inter }}^{2}
$$

such that

$$
\sum_{\mathrm{n}=1}^{\mathrm{N}}<\mathrm{q}_{\mathrm{n}}>_{\text {Intra }}^{2}=\mathrm{N}\left(<\mathrm{q}>_{\text {Inter }}^{2}+\sigma_{\mathrm{q} \text { Inter }}^{2}\right)=\mathrm{N}<\mathrm{q}>_{\text {Inter }}^{2}\left(1+\mathrm{CV}_{\mathrm{q} \text { Inter }}^{2}\right)
$$

Substitution of eq. A17 into eq. A15 finally yields the variance of the overall response amplitude 


$$
\operatorname{Var}_{\mathrm{i}}=\mathrm{Np}_{\mathrm{Oi}} \mathrm{p}_{\mathrm{Ai}}<\mathrm{q}_{\mathrm{i}}>_{\text {Inter }}^{2}\left(1+\mathrm{CV}_{\mathrm{q} \text { Inter }}^{2}\right)\left[1+\mathrm{CV}_{\mathrm{q} \text { Intra }}^{2}-\mathrm{p}_{\mathrm{Oi}} \mathrm{p}_{\mathrm{Ai}}\right]
$$

The general covariance between the amplitudes at stimulus $i$ and $j$ is the sum of eq. A10 over all sites

$$
\operatorname{Cov}_{\mathrm{i}, \mathrm{j}}=\sum_{\mathrm{n}=1}^{\mathrm{N}} \mathrm{p}_{\mathrm{Oii}} \mathrm{p}_{\mathrm{Ai}} \mathrm{p}_{\mathrm{oj}}\left(\mathrm{p}_{\mathrm{Aji}}-\mathrm{p}_{\mathrm{Aj}}\right)<\mathrm{q}_{\mathrm{n}, \mathrm{i}}><\mathrm{q}_{\mathrm{n}, \mathrm{i}}>
$$

Again, because of the assumed absence of heterogeneity in $\mathrm{p}_{0}$, uniform average occupancy $\mathrm{p}_{\mathrm{A}}$ and uniform average refilling probability this simplifies to

$$
\operatorname{Cov}_{\mathrm{i}, \mathrm{j}}=\mathrm{p}_{\mathrm{O} i \mathrm{i}} \mathrm{p}_{\mathrm{Ai}} \mathrm{p}_{\mathrm{Oj}}\left(\mathrm{p}_{\mathrm{Aj \textrm {i }}}-\mathrm{p}_{\mathrm{Aj}}\right) \sum_{\mathrm{n}=1}^{\mathrm{N}}<\mathrm{q}_{\mathrm{n}, \mathrm{i}}><\mathrm{q}_{\mathrm{n}, \mathrm{j}}>
$$

In case of intersite quantal variability, intrasite quantal size averages at different stimulus number are of course correlated relative to the intersite quantal size mean. This correlation has to be considered when substituting the mean of the product into the product of the means

$$
\operatorname{Cov}_{\mathrm{i}, \mathrm{j}}=\mathrm{p}_{\mathrm{Oi}} \mathrm{p}_{\mathrm{Ai}} \mathrm{p}_{\mathrm{O} j}\left(\mathrm{p}_{\mathrm{Aj \textrm {ji }}}-\mathrm{p}_{\mathrm{Aj}}\right)\left(<\mathrm{q}_{\mathrm{i}}>_{\text {Iner }}<\mathrm{q}_{\mathrm{j}}>_{\text {Inter }}+\operatorname{cov}\left(<\mathrm{q}_{\mathrm{n}, \mathrm{i}}>,<\mathrm{q}_{\mathrm{n}, \mathrm{j}}>\right)\right.
$$

A worst case estimate for the covariance term is obtained assuming that no change occurs in quantal size average and distribution between stimuli, then

$$
\operatorname{cov}\left(<\mathrm{q}_{\mathrm{n}, \mathrm{i}}>,<\mathrm{q}_{\mathrm{n}, \mathrm{j}}>\right) \leq \sigma_{\mathrm{q} \text { tnter }}^{2}
$$

and the quantal size covariance term can be accounted for by a correction factor $\left(1+\mathrm{CV}_{\mathrm{qI} \text { Inter }}^{2}\right)$

$$
\operatorname{Cov}_{\mathrm{i}, \mathrm{j}}=\mathrm{p}_{\mathrm{oi}} \mathrm{p}_{\mathrm{Ai}_{\mathrm{i}}} \mathrm{p}_{\mathrm{oj}}\left(\mathrm{p}_{\mathrm{Aji \textrm {i }}}-\mathrm{p}_{\mathrm{oj}}\right)<\mathrm{q}_{\mathrm{i}}>_{\text {Inter }}<\mathrm{q}_{\mathrm{j}}>_{\text {Inter }}\left(1+\mathrm{CV}_{\text {qInter }}^{2}\right)
$$

The expressions including quantal size variability are now combined to the expressions for estimating the parameters of interest in analogy to the model paragraph in the methods chapter:

a) Combining mean (see eq. A13) and variance (see eq. A18) yields the classical parabolic variance-mean relationship:

$$
\operatorname{Var}_{\mathrm{i}}=<\mathrm{q}>_{\text {Inter }}\left(1+\mathrm{CV}_{\mathrm{q} \text { Intra }}^{2}\right)\left(1+\mathrm{CV}_{\text {qIner }}^{2}\right) \overline{\mathrm{Y}}_{\mathrm{i}}-\frac{1}{\mathrm{~N}}\left(1+\mathrm{CV}_{\mathrm{q} \text { mere }}^{2}\right) \overline{\mathrm{Y}}_{\mathrm{i}}^{2}
$$

Dividing eq. A24 by the mean yields a linear relationship between variance over mean ratio versus mean:

$$
\frac{\operatorname{Var}_{\mathrm{i}}}{\overline{\mathrm{Y}}_{\mathrm{i}}}=<\mathrm{q}>_{\text {Inter }}\left(1+\mathrm{CV}_{\mathrm{q} \text { Intra }}^{2}\right)\left(1+\mathrm{CV}_{\mathrm{q} \text { Inter }}^{2}\right)-\frac{1}{\mathrm{~N}}\left(1+\mathrm{CV}_{\mathrm{qInter}}^{2}\right) \overline{\mathrm{Y}}_{\mathrm{i}}
$$


b) Calculation of $\mathrm{N}$ from the mean and covariance of consecutive EPSCs in case of effectively no refilling, i.e. $b_{i j j}=0$. It follows from eq. A23

$$
\operatorname{Cov}_{\mathrm{i}, \mathrm{j}}=-\mathrm{Np}_{\mathrm{Oi}} \mathrm{p}_{\mathrm{Ai}} \mathrm{p}_{\mathrm{Oj}} \mathrm{p}_{\mathrm{Aj}}<\mathrm{q}_{\mathrm{i}}>_{\text {Inter }}<\mathrm{q}_{\mathrm{j}}>_{\text {Inter }}\left(1-\mathrm{CV}_{\mathrm{q} \text { Inter }}^{2}\right)
$$

Thus dividing the negative product of the mean amplitudes of consecutive EPSCs (see eq. A13) by the covariance between them (see eq. A26) yields the number of release sites

$$
\mathrm{N}=-\frac{\overline{\mathrm{Y}}_{\mathrm{i}} \overline{\mathrm{Y}}_{\mathrm{i}+1}}{\operatorname{Cov}_{\mathrm{i}, \mathrm{i}+1}}\left(1+\mathrm{CV}_{\mathrm{q} \text { Inter }}^{2}\right)^{-1}
$$

Calculation of $\mathrm{q}$ for each response in the train from mean, variance and covariance of consecutive EPSCs. In the case of effectively no refillinq an expression for the actual quantal size for each response in the train can be derived from the covariance (see eq. A26), mean and variance (see eq. A13). Division of eq. A26 for consecutive responses $i$ and $i+1$ by eq. A13 for response $i$ or $i+1$ yields

$$
\begin{array}{r}
\frac{\operatorname{Cov}_{\mathrm{i}, \mathrm{i}+1}}{\overline{\mathrm{Y}}_{\mathrm{i}}}=-<\mathrm{q}_{\mathrm{i}+1}>\mathrm{p}_{\mathrm{Oi+1}} \mathrm{p}_{\mathrm{Ai}+1}\left(1+\mathrm{CV}_{\mathrm{qIntra}}^{2}\right) \\
\text { and } \frac{\operatorname{Cov}_{\mathrm{i}, \mathrm{i}+1}}{\overline{\mathrm{Y}}_{\mathrm{i}+1}}=-<\mathrm{q}_{\mathrm{i}}>\mathrm{p}_{\mathrm{Oi}} \mathrm{p}_{\mathrm{Ai}}\left(1+\mathrm{CV}_{\mathrm{q} \text { Intra }}^{2}\right)
\end{array}
$$

Substitution of eq. A13 into eq. A25 and writing it for response i and i+1 gives

$$
\frac{\operatorname{Var}_{i}}{\bar{Y}_{i}}=<q_{i}>_{\text {Inter }}\left(1+C V_{\text {qIntra }}^{2}\right)\left(1+C_{\text {qInter }}^{2}\right)-<q_{i}>\left(1+\mathrm{CV}_{\text {qInter }}^{2}\right) p_{O i} p_{A i}
$$

and

$$
\frac{\operatorname{Var}_{i+1}}{\bar{Y}_{i+1}}=<q_{i+1}>_{\text {Inter }}\left(1+C_{\text {qIntra }}^{2}\right)\left(1+C_{\text {qInter }}^{2}\right)-<q_{i+1}>\left(1+C_{\text {qInter }}^{2}\right) p_{\text {Oi+1 }} p_{\text {Ai+1 }}(A 29 b)
$$

In order to distinguish the approach including the covariance form the approach based only on mean and variance the quantal size measure is now defined as $\mathrm{q}_{\mathrm{i}}{ }^{* *} \equiv\left\langle\mathrm{q}_{\mathrm{j}}{ }^{2}\right\rangle /\left\langle\mathrm{q}_{\mathrm{j}}\right\rangle$ (compare to eq. M14, M16). Solving eq. A29a and A29b for $\left\langle\mathrm{q}_{\mathrm{j}}{ }^{2}\right\rangle \mid\left\langle\mathrm{q}_{\mathrm{j}}\right\rangle=\mathrm{q}_{\mathrm{i}}{ }^{* *}$ with $\mathrm{j}=\mathrm{i}$ or $\mathrm{j}=\mathrm{i}+1$ respectively and substituting eq. A28b and A28a, respectively, yields

$$
<\mathrm{q}_{\mathrm{i}}>_{\text {Inter }}\left(1+\mathrm{CV}_{\mathrm{q} \text { Intra }}^{2}\right)\left(1+\mathrm{CV}_{\mathrm{qInter}}^{2}\right)=\frac{\mathrm{Var}_{\mathrm{i}}}{\overline{\mathrm{Y}}_{\mathrm{i}}}-\frac{\mathrm{CoV}_{\mathrm{i}, \mathrm{i}+1}}{\overline{\mathrm{Y}}_{\mathrm{i}, \mathrm{i}+1}}
$$

and 


$$
<\mathrm{q}_{\mathrm{i}+1}>_{\text {Inter }}\left(1+\mathrm{CV}_{\text {qunta }}^{2}\right)\left(1+\mathrm{CV}_{\text {qInter }}^{2}\right)=\frac{\mathrm{Var}_{\mathrm{i}+1}}{\overline{\mathrm{Y}}_{\mathrm{i}+1}}-\frac{\mathrm{CoV}_{\mathrm{i}, \mathrm{i}+1}}{\overline{\mathrm{Y}}_{\mathrm{i}}}
$$

Now setting $\mathrm{i}+1$ in eq. A30b back to $\mathrm{i}$ yields two independent equations for $\mathrm{q}_{\mathrm{i}}{ }^{* *}$

$$
<\mathrm{q}_{\mathrm{i}}>_{\text {Inter }}\left(1+\mathrm{C}_{\mathrm{qInta}}^{2}\right)\left(1+\mathrm{CV}_{\mathrm{q} \text { Inter }}^{2}\right)=\frac{\operatorname{Var}_{\mathrm{i}}}{\overline{\mathrm{Y}}_{\mathrm{i}}}-\frac{\operatorname{Cov}_{\mathrm{i}-1, \mathrm{i}}}{\overline{\mathrm{Y}}_{\mathrm{i}-1}}
$$

Applying these equations to estimate the quantal size for each respose in the train eq. A30a was used for response 1, the mean of eq. A30a and A30c for responses 2 to 4 and eq. A30c for response 5 . 


\section{B. Parameters applied in the simulations}

\begin{tabular}{|c|c|c|c|}
\hline $\begin{array}{c}\text { parameter } \\
\text { (symbol) }\end{array}$ & value & unit & reference \\
\hline $\mathrm{F}$ & 9 & - & (Meyer 1999) \\
\hline $\mathrm{R}$ & 0.15 & - & free parameter \\
\hline$\tau_{\mathrm{p} 1}$ & 0.3 & $\mathrm{~s}$ & (Wu and Borst 1999) \\
\hline$\tau_{\mathrm{p} 2}$ & 5.2 & $\mathrm{~s}$ & (Trommershäuser 2000) \\
\hline $\mathrm{J}_{\mathrm{Ca}, \max }$ & 2.615 & - & (Schneggenburger et al. 1999) \\
\hline $\mathrm{EC}_{50}$ & 2.617 & $\mathrm{mM}$ & (Schneggenburger et al. 1999) \\
\hline $\mathrm{K}_{1 / 2}$ & 3.38 & $\mu \mathrm{M}$ & free parameter \\
\hline$\eta$ & 2.734 & - & free parameter \\
\hline$\gamma$ & 0.249 & - & free parameter \\
\hline $\mathrm{K}_{\mathrm{D}}$ & 0.0825 & $\mu \mathrm{M}$ & free parameter \\
\hline$\left[\mathrm{Ca}^{2+}\right]_{\mathrm{r}}$ & 0.1 & $\mu \mathrm{M}$ & (Helmchen et al. 1997) \\
\hline $\mathrm{x}_{0}$ & 0.4 & $\mu \mathrm{M}$ & free parameter, (Helmchen et al. 1997) \\
\hline$\tau$ & 0.15 & $\mathrm{~s}$ & free parameter, (Helmchen et al. 1997) \\
\hline
\end{tabular}

Table 7: Parameters applied in the simulation of the three-state pool model and the calculation of release probabilities during facilitation. For the detailed documentation refer to Trommershäuser (2000). 


\section{Calculation of the uncertainty of the segmented estimation of the variance}

In a data set of size $\mathrm{N}$, record $\mathrm{i}$ may be represented as the sum of its expectation independent of $\mathrm{i}$ and a fluctuating term with zero mean

$$
\mathrm{x}_{\mathrm{i}}=\mathrm{x}_{0}+\delta \mathrm{x}_{\mathrm{i}}
$$

with expectation

$$
\mathrm{E}\left(\mathrm{x}_{\mathrm{i}}\right)=\mathrm{x}_{0}
$$

and variance

$$
\mathrm{V}\left(\mathrm{x}_{\mathrm{i}}\right)=\mathrm{V}\left(\delta \mathrm{x}_{\mathrm{i}}\right)=\sigma^{2}
$$

Assuming the fluctuating term to be a Gaussian stochastic variable with zero mean in case that all $\delta \mathrm{x}_{\mathrm{i}}$ and $\delta \mathrm{x}_{\mathrm{j}}$ are statistically independent the following equations hold (see Heinemann and Conti 1992)

$$
\begin{aligned}
<\delta x_{\mathrm{i}} \delta \mathrm{x}_{\mathrm{j}}>= & <\delta \mathrm{x}_{\mathrm{i}}><\delta \mathrm{x}_{\mathrm{j}}>=0 ; \quad \mathrm{i} \neq \mathrm{j} \\
<\delta \mathrm{x}_{\mathrm{i}}^{2}> & =\sigma^{2} \\
<\delta \mathrm{x}_{\mathrm{i}}^{2} \delta \mathrm{x}_{\mathrm{j}}^{2}>= & <\delta \mathrm{x}_{\mathrm{i}}^{2}><\delta \mathrm{x}_{\mathrm{j}}^{2}>=\sigma^{4} ; \quad \mathrm{i} \neq \mathrm{j} \\
<\delta \mathrm{x}_{\mathrm{i}}^{4}> & =3 \sigma^{4}
\end{aligned}
$$

Mean and variance are determined for segment $i$ of size $\mathrm{R}$ by

$$
\bar{X}_{i}=\frac{1}{R} \sum_{j=n(i, R)}^{n(i, R)+R-1} X_{j}
$$

and

$$
V_{i}=\frac{1}{R-1} \sum_{j=n(i, R)}^{n(i, R)+R-1}\left(x_{j}-\bar{x}_{i}\right)^{2}
$$

respectively, where $n(i, R)$ is the first entry of segment $i$.

The overall variance estimate is then given as the average segment variance

$$
\mathrm{v}_{\text {tot }}=\overline{\mathrm{v}}_{\mathrm{i}}
$$

and the uncertainty in this estimate can be calculated by its variance as

$$
\operatorname{Var}\left(\mathrm{v}_{\text {tot }}\right)=<\mathrm{v}_{\text {tot }}^{2}>-<\mathrm{v}_{\text {tot }}>^{2}
$$

Now three different possibilities of choosing the segments with respect to their size and mutual overlap are examined regarding the uncertainty of the variance estimate 
they yield. These possibilities are non-overlapping independent segments, segments, which overlap by half the number of entries, and segments, which are shifted along the entries of the data set in steps of one entry. As measure for the uncertainty of the estimate eq. C13 is analysed for each case.

i) Non-overlapping independent segments:

Segments of size $\mathrm{R}$, data set of $\mathrm{N}$ records ( $\mathrm{N}$ should be a multiple of $\mathrm{R}$ )

$$
\mathrm{v}_{\text {tot }}=\frac{1}{\mathrm{~N} / \mathrm{R}} \sum_{\mathrm{i}=1}^{\mathrm{N} / \mathrm{R}} \mathrm{v}_{\mathrm{i}}
$$

thus

$$
\mathrm{v}_{\text {tot }}^{2}=\frac{1}{(\mathrm{~N} / \mathrm{R})^{2}}\left[\sum_{\mathrm{i}=1}^{\mathrm{N} / \mathrm{R}} \mathrm{v}_{\mathrm{i}}\right]^{2}
$$

and the expectations of these are

$$
\begin{aligned}
& \left.<\mathrm{v}_{\text {tot }}>=\frac{1}{\mathrm{~N} / \mathrm{R}} \sum_{\mathrm{i}=1}^{\mathrm{N} / \mathrm{R}}\left\langle\mathrm{v}_{\mathrm{i}}\right\rangle=<\mathrm{v}_{\mathrm{i}}\right\rangle \\
& <\mathrm{v}_{\text {tot }}^{2}>=\frac{1}{(\mathrm{~N} / \mathrm{R})^{2}}[\sum_{\mathrm{i}=1}^{\mathrm{N} / \mathrm{R}}<\mathrm{v}_{\mathrm{i}}^{2}>+2 \sum_{\mathrm{i}=1}^{\mathrm{N} / \mathrm{R}} \sum_{\mathrm{j}=\mathrm{i}+1}^{\mathrm{N} / \mathrm{R}} \underbrace{\left.<\mathrm{v}_{\mathrm{i}} \mathrm{V}_{\mathrm{j}}\right\rangle}_{\text {non-overlapping }}]
\end{aligned}
$$

with $\left\langle\mathrm{v}_{\mathrm{i}} \mathrm{v}_{\mathrm{j}}\right\rangle=\left\langle\mathrm{v}_{\mathrm{i}}\right\rangle\left\langle\mathrm{v}_{\mathrm{j}}\right\rangle=\left\langle\mathrm{v}_{\mathrm{i}}\right\rangle^{2}$, because non-overlapping segments are independent

$$
\left.<\mathrm{v}_{\text {tot }}^{2}>=\frac{1}{\mathrm{~N} / \mathrm{R}}\left[\left\langle\mathrm{v}_{\mathrm{i}}^{2}\right\rangle+\left(\frac{\mathrm{N}}{\mathrm{R}}-1\right)<\mathrm{v}_{\mathrm{i}}\right\rangle^{2}\right]
$$

Then substituting eq. $\mathrm{C} 15$ and eq. $\mathrm{C} 17$ into eq. $\mathrm{C} 13$ yields

$$
\operatorname{Var}\left(\mathrm{v}_{\text {tot }}\right)=\frac{1}{\mathrm{~N} / \mathrm{R}}\left(<\mathrm{v}_{\mathrm{i}}^{2}>-<\mathrm{v}_{\mathrm{i}}>^{2}\right)
$$

Now $\left\langle\mathrm{v}_{\mathrm{i}}\right\rangle$ and $\left\langle\mathrm{v}_{\mathrm{i}}{ }^{2}\right\rangle$ have to be determined: Substitution of eq. $\mathrm{C} 1$ and eq. $\mathrm{C} 8$ into eq. C9 and rearrangement yield:

$$
V_{i}=\frac{1}{R} \sum_{j=n(i, R)}^{n(i, R)+R-1} \delta x_{i}^{2}-\frac{2}{R(R-1)} \sum_{j=n(i, R)}^{n(i, R)+R-1} \sum_{k=j+1}^{n(i, R)+R-1} \delta x_{j} \delta x_{k}
$$

Calculation of $\left\langle\mathrm{v}_{\mathrm{i}}\right\rangle$, see equations eq. $\mathrm{C} 4$ and eq. $\mathrm{C} 5$ : 


$$
<\mathrm{v}_{\mathrm{i}}>=\frac{1}{\mathrm{R}} \sum_{\mathrm{j}=\mathrm{n}(\mathrm{i}, \mathrm{R})}^{\mathrm{n}(\mathrm{i}, \mathrm{R})+\mathrm{R}-1} \underbrace{<\delta \mathrm{x}_{\mathrm{i}}^{2}>}_{=\sigma^{2}}-\frac{1}{\mathrm{R}(\mathrm{R}-1)} \sum_{\mathrm{j}=\mathrm{n}(\mathrm{i}, \mathrm{R})}^{\mathrm{n}(\mathrm{i}, \mathrm{R})+\mathrm{R}-1} \sum_{\mathrm{k}=1, \mathrm{k} \neq \mathrm{j}}^{\mathrm{n}(\mathrm{i}, \mathrm{R})+\mathrm{R}-1} \underbrace{<\delta \mathrm{x}_{\mathrm{j}} \delta \mathrm{x}_{\mathrm{k}}>}_{=0}
$$

thus

$$
<\mathrm{v}_{\mathrm{i}}>=\sigma^{2}
$$

as expected. From eq. C19 follows

$$
\begin{gathered}
v_{i}^{2}=\frac{1}{R} \sum_{j=n(i, R)}^{n(i, R)+R-1} \sum_{k=n(i, R)}^{n(i, R)+R-1} \delta x_{j}^{2} \delta x_{k}^{2}-\frac{4}{R^{2}(R-1)} \sum_{j=n(i, R)}^{n(i, R)+R-1} \sum_{k=n(i, R)}^{n(i, R)+R-1} \sum_{l=k+1}^{n(i, R)+R-1} \delta x_{j}^{2} \delta x_{k} \delta x_{1} \\
+\frac{4}{R^{2}(R-1)^{2}}\left(\sum_{j=n(i, R)}^{n(i, R)+R-1} \sum_{k=j+1}^{n(i, R)+R-1} \delta x_{j} \delta x_{k}\right)^{2}
\end{gathered}
$$

Calculating the expectation $\left\langle\mathrm{v}_{\mathrm{i}}{ }^{2}\right\rangle$ the first and the last sum only contribute non-zero terms, see eq. $\mathrm{C} 4$ until eq. $\mathrm{C} 7$ :

$$
\begin{aligned}
<v_{i}^{2}>= & \frac{1}{R^{2}} \sum_{j=i}^{i+R-1}<\delta x_{j}^{4}>+\frac{2}{R^{2}} \sum_{j=i}^{i+R-1} \sum_{k=j+1}^{i+R-1}<\delta x_{j}^{2} \delta x_{k}^{2}> \\
& +\frac{4}{R^{2}(R-1)^{2}} \sum_{j=i}^{i+R-1} \sum_{k=j+1}^{i+R-1}<\delta x_{j}^{2} \delta x_{k}^{2}> \\
& =\frac{3}{R} \sigma^{4}+\left(\frac{2}{R^{2}}+\frac{4}{R^{2}(R-1)^{2}}\right) \frac{R(R-1)}{2} \sigma^{4}
\end{aligned}
$$

finally

$$
<\mathrm{v}_{\mathrm{i}}^{2}>=\frac{\mathrm{R}+1}{\mathrm{R}-1} \sigma^{4}
$$

Substituting eq. C20 and eq. C22 into eq. C18 yields the uncertainty in the variance estimate for non-overlapping segments of size R:

$$
\operatorname{Var}\left(\mathrm{v}_{\text {tot }}\right)=\frac{2 \mathrm{R}}{\mathrm{N}(\mathrm{R}-1)} \sigma^{4}
$$

The results for $\left\langle v_{i}\right\rangle$ and $\left\langle v_{i}{ }^{2}\right\rangle$ (eq. C20 and eq. C22) in this section hold generally for any data segment of size $\mathrm{R}$. 
ii) Half-overlapping segments:

Segments of size $\mathrm{R}$, data set of $\mathrm{N}$ records ( $\mathrm{N}$ should be a multiple of $\mathrm{R}, \mathrm{R}$ a multiple of 2)

$$
\mathrm{V}_{\mathrm{tot}}=\frac{1}{(2 \mathrm{~N} / \mathrm{R}-1)} \sum_{\mathrm{i}=1}^{2 \mathrm{~N} / \mathrm{R}-1} \mathrm{v}_{\mathrm{i}}
$$

and thus

$$
\mathrm{V}_{\text {tot }}^{2}=\frac{1}{(2 \mathrm{~N} / \mathrm{R}-1)^{2}}[\sum_{\mathrm{i}=1}^{2 \mathrm{~N} / \mathrm{R}-1} \mathrm{~V}_{\mathrm{i}}^{2}+2 \sum_{\mathrm{i}=1}^{2 \mathrm{~N} / \mathrm{R}-2} \underbrace{\mathrm{V}_{\mathrm{i}} \mathrm{V}_{\mathrm{i}+1}}_{\text {overlapping }}+2 \sum_{\mathrm{i}=1}^{2 \mathrm{~N} / \mathrm{R}-1} \sum_{\mathrm{j}=\mathrm{i}+2}^{2 \mathrm{~N} / \mathrm{R}-1} \underbrace{\mathrm{V}_{\mathrm{i}} \mathrm{V}_{\mathrm{j}}}_{\text {non-overlapping }}]
$$

The expectations of these are

$$
<\mathrm{v}_{\text {tot }}>=<\mathrm{v}_{\mathrm{i}}>
$$

as above in eq. C20, and

$$
<\mathrm{v}_{\text {tot }}^{2}>=\frac{1}{2 \mathrm{~N} / \mathrm{R}-1}<\mathrm{v}_{\mathrm{i}}^{2}>+\frac{4(\mathrm{~N} / \mathrm{R}-1)}{(2 \mathrm{~N} / \mathrm{R}-1)^{2}} \underbrace{<\mathrm{v}_{\mathrm{i}} \mathrm{v}_{\mathrm{i}+1}>}_{\text {half-overlapping }}+\frac{2(2 \mathrm{~N} / \mathrm{R}-3)}{2 \mathrm{~N} / \mathrm{R}-1} \underbrace{<\mathrm{v}_{\mathrm{i}} \mathrm{v}_{\mathrm{j}}>}_{\substack{\text { non-overlapping } \\=<\mathrm{V}_{\mathrm{i}}>^{2}}}
$$

Substituting eq. C26 and eq. C27 into eq. C13 yields:

$$
\operatorname{Var}\left(\mathrm{v}_{\text {tot }}\right)=\frac{1}{(2 \mathrm{~N} / \mathrm{R}-1)}<\mathrm{v}_{\mathrm{i}}^{2}>+\frac{4(\mathrm{~N} / \mathrm{R}-1)}{(2 \mathrm{~N} / \mathrm{R}-1)^{2}} \underbrace{<\mathrm{v}_{\mathrm{i}} \mathrm{v}_{\mathrm{i}+1}>}_{\text {half-overlapping }}+\frac{6 \mathrm{~N} / \mathrm{R}-5}{2 \mathrm{~N} / \mathrm{R}-1}<\mathrm{v}_{\mathrm{i}}>^{2}
$$

Now calculate $\left\langle\mathrm{v}_{\mathrm{i}} \mathrm{v}_{\mathrm{i}+1}\right\rangle$ for half overlapping segments:

$$
V_{i}=\frac{1}{R} \sum_{j=\frac{R}{2}(i-1)+1}^{\frac{R}{2}(i+1)} \delta x_{j}^{2}-\frac{2}{R(R-1)} \sum_{j=\frac{R}{2}(i-1)+1}^{\frac{R}{2}(i+1)} \sum_{k=j+1}^{\frac{R}{2}(i+1)} \delta x_{j} \delta x_{k}
$$

$\mathrm{R} / 2$ of the entries in the sums $\mathrm{v}_{\mathrm{i}}$ and $\mathrm{v}_{\mathrm{i}+1}$ occur in both. Calculating the expectation of the product of $v_{i}$ and $v_{i+1}$ only the products of their first sums and their second sums contribute non-zero terms, see eq. C4 until eq. C7:

$$
\begin{gathered}
<\mathrm{v}_{\mathrm{i}} \mathrm{v}_{\mathrm{i}+1}>=\frac{1}{\mathrm{R}^{2}}\left[\mathrm{R} / 2<\delta \mathrm{x}_{\mathrm{i}}^{4}>+\mathrm{R} / 2(\mathrm{R}-1)<\delta \mathrm{x}_{\mathrm{i}}^{2} \delta \mathrm{x}_{\mathrm{j}}^{2}>+\mathrm{R}^{2} / 2<\delta \mathrm{x}_{\mathrm{i}}^{2} \delta \mathrm{x}_{\mathrm{j}}^{2}>\right] \\
+\frac{4}{\mathrm{R}^{2}(\mathrm{R}-1)^{2}}\left[\mathrm{R} / 4(\mathrm{R} / 2-1)<\delta \mathrm{x}_{\mathrm{i}}^{2} \delta \mathrm{x}_{\mathrm{j}}^{2}>\right] ; \quad \mathrm{i} \neq \mathrm{j} \quad
\end{gathered}
$$

To give with eq. C6 and eq. C7: 


$$
<\mathrm{v}_{\mathrm{i}} \mathrm{v}_{\mathrm{i}+1}>=\left[1+\frac{1}{\mathrm{R}}+\frac{\mathrm{R} / 2-1}{\mathrm{R}(\mathrm{R}-1)^{2}}\right] \sigma^{4}
$$

Thus finally by substitution of eq. C20, eq. C22 and eq. C3 into eq. C28

$$
\operatorname{Var}\left(\mathrm{v}_{\mathrm{tot}}\right)=\left[\frac{(\mathrm{R}+1)}{(2 \mathrm{~N} / \mathrm{R}-1)(\mathrm{R}-1)}+4 \frac{(\mathrm{N} / \mathrm{R}-1)}{(2 \mathrm{~N} / \mathrm{R}-1)^{2}}\left(1+\frac{1}{\mathrm{R}}+\frac{(\mathrm{R} / 2-1)}{\mathrm{R}(\mathrm{R}-1)^{2}}\right)-\frac{(6 \mathrm{~N} / \mathrm{R}-5)}{(2 \mathrm{~N} / \mathrm{R}-1)}\right] \sigma^{4}
$$

iii) Totally overlapping segments:

Segments of size $\mathrm{R}$, data set of $\mathrm{N}$ records

$$
\begin{gathered}
\mathrm{v}_{\mathrm{tot}}=\frac{1}{(\mathrm{~N}-\mathrm{R}+1)} \sum_{\mathrm{i}=1}^{\mathrm{N}-\mathrm{R}+1} \mathrm{v}_{\mathrm{i}} \\
\mathrm{v}_{\mathrm{tot}}^{2}=\frac{1}{(\mathrm{~N}-\mathrm{R}+1)^{2}}\left[\sum_{\mathrm{i}=1}^{\mathrm{N}-\mathrm{R}+1} \mathrm{v}_{\mathrm{i}}^{2}+2 \sum_{\mathrm{i}=1}^{\mathrm{N}-\mathrm{R}+1} \sum_{\mathrm{j}=\mathrm{i}+1}^{\mathrm{N}-\mathrm{R}+1} \mathrm{v}_{\mathrm{i}} \mathrm{v}_{\mathrm{j}}\right]
\end{gathered}
$$

The expectations of these are

$$
<\mathrm{v}_{\text {tot }}>=<\mathrm{v}_{\mathrm{i}}>
$$

and for $\left\langle\mathrm{v}^{2}{ }_{\text {tot }}\right\rangle$ two cases must be distinguished:

a) All segments overlap with any other if $\mathrm{R} \geq(\mathrm{N}+1) / 2$ (first segment ends at $i_{\text {end }}=R$, last segment starts at $i_{\text {start }}=N-R+1$ )

$$
\begin{gathered}
\mathrm{v}_{\text {tot }}^{2}=\frac{1}{(\mathrm{~N}-\mathrm{R}+1)^{2}}[\sum_{\mathrm{i}=1}^{\mathrm{N}-\mathrm{R}+1} \mathrm{v}_{\mathrm{i}}^{2}+2 \sum_{\mathrm{i}=1}^{\mathrm{N}-\mathrm{R}+1} \sum_{\mathrm{n}=1}^{\mathrm{N}-\mathrm{R}} \underbrace{\mathrm{V}_{\mathrm{i}} \mathrm{v}_{\mathrm{i}+\mathrm{n}}}_{\text {overlapping }}] \\
<\mathrm{v}_{\text {tot }}^{2}>=\frac{1}{(\mathrm{~N}-\mathrm{R}+1)}<\mathrm{v}_{\mathrm{i}}^{2}>+2 \sum_{\mathrm{n}=1}^{\mathrm{N}-\mathrm{R}} \frac{(\mathrm{N}-\mathrm{R}+1-\mathrm{n})}{(\mathrm{N}-\mathrm{R}+1)^{2}}<\mathrm{v}_{\mathrm{i}} \mathrm{v}_{\mathrm{i}+\mathrm{n}}>
\end{gathered}
$$

b) Not all segments overlap with any other $\mathrm{R}<(\mathrm{N}+1) / 2$

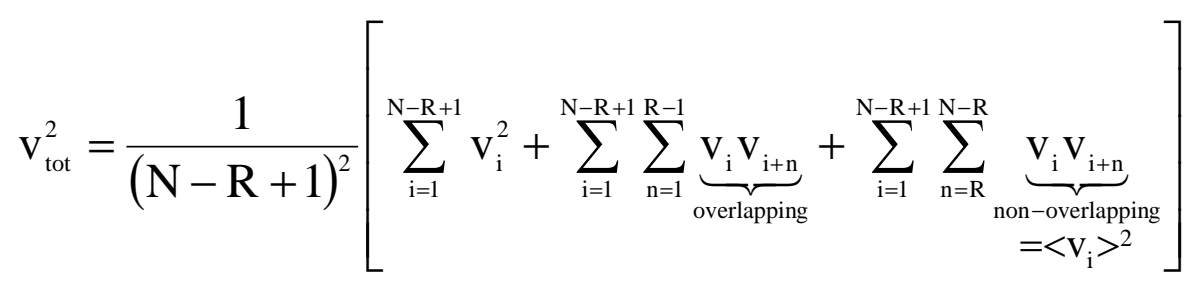




$$
\begin{aligned}
& <\mathrm{v}_{\text {tot }}^{2}>=\frac{1}{(\mathrm{~N}-\mathrm{R}+1)^{2}}<\mathrm{v}_{\mathrm{i}}^{2}>+2 \sum_{\mathrm{n}=1}^{\mathrm{R}-1} \frac{(\mathrm{N}-\mathrm{R}+1-\mathrm{n})}{(\mathrm{N}-\mathrm{R}+1)^{2}}<\mathrm{v}_{\mathrm{i}} \mathrm{v}_{\mathrm{i}+\mathrm{n}}> \\
& +\frac{(\mathrm{N}-2 \mathrm{R}+1)(\mathrm{N}-2 \mathrm{R}+2)}{(\mathrm{N}-\mathrm{R}+1)^{2}}<\mathrm{v}_{\mathrm{i}}>^{2}
\end{aligned}
$$

Now calculate $\left\langle\mathrm{v}_{\mathrm{i}} \mathrm{v}_{\mathrm{i}+\mathrm{n}}\right\rangle$ :

$$
V_{i}=\frac{1}{R} \sum_{j=i}^{i+R-1} \delta x_{j}^{2}-\frac{2}{R(R-1)} \sum_{j=i}^{i+R-1} \sum_{k=j+1}^{i+R-1} \delta x_{j} \delta x_{k}
$$

Define the number of entries contained in both $\mathrm{v}_{\mathrm{i}}$ and $\mathrm{v}_{\mathrm{i}+\mathrm{n}}$, which is

$$
\mathrm{X}_{\mathrm{n}} \equiv \mathrm{R}-\mathrm{n}
$$

As in the above sections again only the products of the first sum of $v_{i}$ with the first sum of $v_{i+n}$ and the second sum of $v_{i}$ with the second sum of $v_{i+n}$ yield non-zero terms for the expectation:

$$
\begin{gathered}
<\mathrm{V}_{\mathrm{i}} \mathrm{V}_{\mathrm{i}+\mathrm{n}}>=\frac{1}{\mathrm{R}^{2}}\left[\mathrm{X}_{\mathrm{n}}<\delta \mathrm{x}_{\mathrm{i}}^{4}>+\mathrm{X}_{\mathrm{n}}(\mathrm{R}-1)<\delta \mathrm{x}_{\mathrm{i}}^{2} \delta \mathrm{x}_{\mathrm{j}}^{2}>+\left(\mathrm{R}-\mathrm{X}_{\mathrm{n}}\right) \mathrm{R}<\delta \mathrm{x}_{\mathrm{i}}^{2} \delta \mathrm{x}_{\mathrm{j}}^{2}>\right] \\
+\frac{4}{\mathrm{R}^{2}(\mathrm{R}-1)^{2}}\left[\mathrm{X}_{\mathrm{n}}\left(\mathrm{X}_{\mathrm{n}}-1\right) / 2<\delta \mathrm{x}_{\mathrm{i}}^{2} \delta \mathrm{x}_{\mathrm{j}}^{2}>\right] ; \quad \mathrm{i} \neq \mathrm{j}
\end{gathered}
$$

After rearrangement and substitution of eq. C41

$$
<\mathrm{v}_{\mathrm{i}} \mathrm{v}_{\mathrm{i}+\mathrm{n}}>=\left[1+\frac{2}{\mathrm{R}}-\frac{2 \mathrm{n}}{\mathrm{R}^{2}}+2 \frac{(\mathrm{R}-\mathrm{n})(\mathrm{R}-\mathrm{n}-1)}{\mathrm{R}^{2}(\mathrm{R}-1)^{2}}\right] \sigma^{4}
$$

Now all expressions are derived (eq. C6, eq. C7, eq. C20, eq. C22, eq. C37, eq. C39, eq. C42), required for substitution into eq. 13, which was

$$
\operatorname{Var}\left(\mathrm{v}_{\text {tot }}\right)=<\mathrm{v}_{\text {tot }}^{2}>-<\mathrm{v}_{\text {tot }}>^{2}
$$

to get variance of the variance estimate in the two cases of totally overlapping segments. The expressions for $\operatorname{Var}\left(\mathrm{v}_{\text {tot }}\right)$ are quite long, and therefore not explicitly given here.

To compare all three possibilities one finds, that in each case the variance of the variance estimate can be written as a product of the fourth power of the intrinsic variance $\sigma$ and a factor, which depends on the size of the data set $\mathrm{N}$, the segment size $\mathrm{R}$ and the method of choice $\mathrm{x}$ (see eq. C23 and eq. C32, for case iii) the explicit expressions are not given): 


$$
\operatorname{Var}\left(\mathrm{v}_{\text {tot }}\right)=\operatorname{factor}_{\mathrm{x}}(\mathrm{N}, \mathrm{R}) \sigma^{4}
$$

From eq. C43 follows for the signal to noise ratio of the total variance estimate

$$
\operatorname{SNR}\left(\mathrm{v}_{\text {tot }}\right)=\frac{\left.<\mathrm{v}_{\text {tot }}\right\rangle}{\sqrt{\operatorname{Var}\left(\mathrm{v}_{\text {tot }}\right)}}=\frac{1}{\sqrt{\text { factor }_{\mathrm{x}}(\mathrm{N}, \mathrm{R})}}
$$

The SNR is plotted in the Fig. 8 for all three possibilities for a range of segment sizes $(2-10)$ at a fixed data set size $(\mathrm{N}=100)$. 


\section{Calculation of the uncertainty of the segmented estimation of the covariance}

Assume a data set containing $\mathrm{N}$ trains of recordings. The calculation is carried out analogous to the calculation for the uncertainty in the variance, with the difference that here only the cases of independent records and the segmental estimation with overlapping windows of size 2 are considered. For two recordings in train i, $X_{i}, Y_{i}$, holds

$$
\begin{array}{llll}
X_{i}=X_{0}+\delta x_{i} \text { with } & \langle X\rangle=X_{0}, & \left\langle\delta x_{i}\right\rangle=0, & \operatorname{Var}\left(X_{i}\right)=\operatorname{Var}\left(\delta x_{i}\right)=\sigma_{x}^{2} \\
Y_{i}=Y_{0}+\delta y_{i} \quad \text { with } & \langle Y\rangle=Y_{0}, & \left\langle\delta y_{i}\right\rangle=0, & \operatorname{Var}\left(Y_{i}\right)=\operatorname{Var}\left(\delta y_{i}\right)=\sigma_{y}^{2}
\end{array}
$$

$\left(\delta x_{i}, \delta y_{i}\right.$ assumed to be Gaussian distributed fluctuations, with $\delta x_{i}, \delta y_{j}$ not independent for $i=j$, such that eq. $\mathrm{C} 4$ until eq. $\mathrm{C} 7$ in appendix $\mathrm{C}$ hold here, too.)

a) In case of $\mathrm{N}$ independent records the formula for the covariance estimation is

$$
\begin{aligned}
& \qquad \begin{array}{l}
\operatorname{cov}_{x, y}=\frac{1}{(N-1)} \sum_{i=1}^{N}\left(X_{i}-\bar{X}\right)\left(Y_{i}-\bar{Y}\right), \\
\text { with } \quad \bar{X}=\frac{1}{N} \sum_{i=1}^{N} X_{i} \text { and } \bar{Y}=\frac{1}{N} \sum_{i=1}^{N} Y_{i}
\end{array}
\end{aligned}
$$

The expected value of this is

$$
\left\langle\operatorname{cov}_{x, y}\right\rangle=\langle\delta x \delta y\rangle
$$

The expected value of the squared covariance estimate is

$$
\left\langle\operatorname{cov}_{x, y}^{2}\right\rangle=\frac{1}{N}\left\langle\delta x^{2} \delta y^{2}\right\rangle+\frac{N-1}{N}\langle\delta x \delta y\rangle^{2}
$$

The variance of the covariance estimation is

$$
\operatorname{Var}\left(\operatorname{cov}_{x, y}\right)=\left\langle\operatorname{cov}_{x, y}^{2}\right\rangle-\left\langle\operatorname{cov}_{x, y}\right\rangle^{2}
$$

Substituting eq. D5 and D6 the variance of the covariance estimation is

$$
\operatorname{Var}\left(\operatorname{cov}_{x, y}\right)=\frac{1}{N}\left(\left\langle\delta x^{2} \delta y^{2}\right\rangle-\langle\delta x \delta y\rangle^{2}\right)
$$

Upper bounds of the terms are on basis of $\sigma_{x}, \sigma_{y}$ and the Schwarz inequality 


$$
\begin{gathered}
\langle\delta x \delta y\rangle^{2} \leq \sigma_{x}^{2} \sigma_{y}^{2} \\
\left\langle\delta x^{2} \delta y^{2}\right\rangle \leq 3 \sigma_{x}^{2} \sigma_{y}^{2}
\end{gathered}
$$

Substituting eq. D9 and D10 into eq. D8 yields an upper bound for the variance of the estimate of the covariance from $\mathrm{N}$ independent records

$$
\operatorname{Var}\left(\operatorname{cov}_{x, y}\right) \leq \frac{3}{N} \sigma_{x}^{2} \sigma_{y}^{2}
$$

b) In the case of $\mathrm{N}$ records analysed segment-wise with overlapping segments of size $\mathrm{R}=2$, the formula for the covariance estimation is

$$
\begin{gathered}
\operatorname{cov}_{\mathrm{x}, \mathrm{y}}=\frac{1}{(\mathrm{~N}-1)} \sum_{\mathrm{i}=1}^{\mathrm{N}-1} \operatorname{cov}_{\mathrm{i}}, \\
\text { with } \operatorname{cov}_{\mathrm{i}}=\sum_{\mathrm{j}=\mathrm{i}}^{\mathrm{i}+1}\left(\mathrm{X}_{\mathrm{j}}-\overline{\mathrm{X}}_{\mathrm{i}}\right)\left(\mathrm{Y}_{\mathrm{j}}-\overline{\mathrm{Y}}_{\mathrm{i}}\right), \\
\text { and } \quad \bar{X}_{i}=\frac{1}{2} \sum_{j=i}^{i+1} X_{j} \text { and } \bar{Y}_{i}=\frac{1}{2} \sum_{j=i}^{i+1} Y_{j}
\end{gathered}
$$

Inserting eq. D14 into eq. D13 gives

$$
\operatorname{cov}_{\mathrm{i}}=\frac{1}{2}\left(\delta \mathrm{x}_{\mathrm{n}}-\delta \mathrm{x}_{\mathrm{n}+1}\right)\left(\delta \mathrm{y}_{\mathrm{n}}-\delta \mathrm{y}_{\mathrm{n}+1}\right)
$$

The uncertainty in the estimate of the covariance expressed as the variance of the estimate is

$$
\operatorname{Var}\left(\operatorname{cov}_{x, y}\right)=<\operatorname{cov}_{x, y}^{2}>-<\operatorname{cov}_{x, y}>^{2}
$$

The expectation of eq. D12 is again

$$
\left\langle\operatorname{cov}_{x, y}\right\rangle=\langle\delta x \delta y\rangle
$$

The square of eq. D12 is

$$
\operatorname{cov}_{x, y}^{2}=\frac{1}{(N-1)^{2}}\left[\sum_{i=1}^{N-1} \operatorname{cov}_{i}^{2}+\sum_{i=1}^{N-1} \operatorname{cov}_{i} \operatorname{cov}_{i+1}+2 \sum_{i=1}^{N-1} \sum_{j=i+2}^{N-1} \operatorname{cov}_{i} \operatorname{cov}_{j}\right]
$$


The expectation of eq. D18

$$
<\operatorname{cov}_{\mathrm{x}, \mathrm{y}}^{2}>=\frac{(\mathrm{N}-1)<\operatorname{cov}_{\mathrm{i}}^{2}>+2(\mathrm{~N}-2)<\operatorname{cov}_{\mathrm{i}} \operatorname{cov}_{\mathrm{i}+1}>+(\mathrm{N}-2)(\mathrm{N}-3)<\operatorname{cov}_{\mathrm{i}}>^{2}}{(\mathrm{~N}-1)^{2}}(\mathrm{D} 19)
$$

The expectation of the square of eq. D15 is

$$
<\operatorname{cov}_{\mathrm{i}}^{2}>=\frac{1}{2}\left(<\delta \mathrm{x}_{\mathrm{i}}^{2} \delta \mathrm{y}_{\mathrm{i}}^{2}>+2<\delta \mathrm{x}_{\mathrm{i}} \delta \mathrm{y}_{\mathrm{i}}>^{2}+<\delta \mathrm{x}_{\mathrm{i}}^{2}><\delta \mathrm{y}_{\mathrm{i}}^{2}>\right)
$$

The expectation of the product of the covariance estimates of two successive segments is

$$
<\operatorname{cov}_{\mathrm{i}} \operatorname{cov}_{\mathrm{i}+1}>=\frac{1}{4}\left(<\delta \mathrm{x}_{\mathrm{i}}^{2} \delta \mathrm{y}_{\mathrm{i}}^{2}>+3<\delta \mathrm{x}_{\mathrm{i}} \delta \mathrm{y}_{\mathrm{i}}>^{2}\right)
$$

Insertion of eq. D17 and D19 and further eq. D20 and D21 into D16 gives the variance of the covariance estimation as

$$
\begin{aligned}
\operatorname{Var}\left(\operatorname{cov}_{\mathrm{x}, \mathrm{y}}\right)= & \frac{1}{2(\mathrm{~N}-1)}<\delta \mathrm{x}_{\mathrm{i}}^{2}><\delta \mathrm{y}_{\mathrm{i}}^{2}>+\frac{2 \mathrm{~N}-3}{2(\mathrm{~N}-1)^{2}}<\delta \mathrm{x}_{\mathrm{i}}^{2} \delta \mathrm{y}_{\mathrm{i}}^{2}> \\
& +\frac{2-\mathrm{N}}{2(\mathrm{~N}-1)^{2}}<\delta \mathrm{x}_{\mathrm{i}} \delta \mathrm{y}_{\mathrm{i}}>^{2}
\end{aligned}
$$

The estimation of upper and lower bounds for this expression, based on $\sigma_{\mathrm{i}}, \sigma_{\mathrm{i}+1}$ as above for $\mathrm{N}>2$ (note that the $3^{\text {rd }}$ term is negative) yields for large values of $\mathrm{N}$ :

$$
0 \leq \operatorname{Var}\left(\operatorname{cov}_{\mathrm{x}, \mathrm{y}}\right) \leq \frac{7 \mathrm{~N}-10}{2(\mathrm{~N}-1)^{2}} \sigma_{\mathrm{x}}^{2} \sigma_{\mathrm{y}}^{2} \approx \frac{3.5}{\mathrm{~N}} \sigma_{\mathrm{x}}^{2} \sigma_{\mathrm{y}}^{2}
$$




\section{E. Noise-analysis in the presence of desensitisation and saturation of postsynaptic receptors}

A recorded EPSC is considered as the sum of the output over of all releases ( $\mathrm{N}$ release sites uniform quantal size $\mathrm{q}$, uniform $\mathrm{p}_{\mathrm{A}}$ and $\mathrm{p}_{0}$ ). The sites are assumed to be independent regarding the release process. Spill over during evoked release is neglected, however spill over from previous release is taken into account. The overall response to a stimulus is

$$
Y_{i}=\sum_{j=1}^{N} q_{i} x_{i j}
$$

The expectation of this is analogous to the calculation presented in the methods part (see eq. M1 until eq. M3):

$$
\bar{Y}_{i}=\sum_{j=1}^{N}<q_{i, j} x_{i j}>=\sum_{j=1}^{N} q_{i j} p_{0 i j} p_{A i j}=N q_{i} p_{0 i} p_{A i}
$$

The covariance between two records at stimulus $\mathrm{i}$ and $\mathrm{j}$ :

$$
\operatorname{Cov}_{\mathrm{ij}}=<\mathrm{Y}_{\mathrm{i}} \mathrm{Y}_{\mathrm{j}}>-\overline{\mathrm{Y}}_{\mathrm{i}} \overline{\mathrm{Y}}_{\mathrm{j}}
$$

Substitution of eq. E1 and E2 yields

$$
\operatorname{Cov}_{\mathrm{ij}}=<\left(\sum_{\mathrm{k}=1}^{\mathrm{N}} \mathrm{q}_{\mathrm{i}} \mathrm{x}_{\mathrm{ik}}\right)\left(\sum_{\mathrm{m}=1}^{\mathrm{N}} \mathrm{q}_{\mathrm{j}} \mathrm{x}_{\mathrm{jm}}\right)>-\mathrm{N}^{2} \mathrm{q}_{\mathrm{i}} \mathrm{p}_{\mathrm{Ai}} \mathrm{p}_{0 \mathrm{i}} \mathrm{q}_{\mathrm{j}} \mathrm{p}_{\mathrm{Aj}} \mathrm{p}_{0 \mathrm{j}}
$$

The expansion of the product of the two sums yields two terms, note $\mathrm{k} \neq \mathrm{m}$

$$
\operatorname{Cov}_{\mathrm{ij}}=\mathrm{N}<\mathrm{q}_{\mathrm{i}} \mathrm{x}_{\mathrm{ik}} \mathrm{q}_{\mathrm{j}} \mathrm{x}_{\mathrm{jk}}>+\mathrm{N}(\mathrm{N}-1)<\mathrm{q}_{\mathrm{i}} \mathrm{x}_{\mathrm{ik}} \mathrm{q}_{\mathrm{j}} \mathrm{x}_{\mathrm{jm}}>-\mathrm{N}^{2} \mathrm{q}_{\mathrm{i}} \mathrm{q}_{\mathrm{j}} \mathrm{p}_{\mathrm{Ai}} \mathrm{p}_{0 \mathrm{i}} \mathrm{p}_{\mathrm{Aj}} \mathrm{p}_{0 \mathrm{j}}
$$

First the expectation of the product of the outcome in response to stimulus $i$ and $j$ at the same release site is considered further. Because the quantal size is assumed to be uniform, $\mathrm{q}_{\mathrm{i}}$ is independent from the rest (see also eq.M3)

$$
<\mathrm{q}_{\mathrm{i}} \mathrm{x}_{\mathrm{ik}} \mathrm{q}_{\mathrm{j}} \mathrm{x}_{\mathrm{jk}}>=\mathrm{q}_{\mathrm{i}}<\mathrm{x}_{\mathrm{ik}} \mathrm{q}_{\mathrm{j}} \mathrm{x}_{\mathrm{jk}}>
$$

Furthermore $\mathrm{x}_{\mathrm{ik}}$ and $\mathrm{x}_{\mathrm{jk}}$ are related as presented in the calculation in the model chapter of the methods part (see eq. M5 - M7). Here, however, it is assumed that previous release interacts with following release events in that it reduces the quantal size of the following event, e.g. such as in the case of desensitisation

$$
<\mathrm{q}_{\mathrm{i}} \mathrm{x}_{\mathrm{ik}} \mathrm{q}_{\mathrm{j}} \mathrm{x}_{\mathrm{jk}}>=\mathrm{q}_{\mathrm{i}} \mathrm{q}_{\mathrm{j}}\left(\mathrm{x}_{\mathrm{i}}\right)<\mathrm{x}_{\mathrm{ik}} \mathrm{x}_{\mathrm{jk}}>=\mathrm{q}_{\mathrm{i}} \mathrm{q}_{\mathrm{j}}\left(\mathrm{x}_{\mathrm{i}}=1\right) \mathrm{p}_{\mathrm{Ai}} \mathrm{p}_{0 \mathrm{i}} \mathrm{p}_{\mathrm{Aj \textrm {j }}} \mathrm{p}_{0 \mathrm{j}}
$$

where $\mathrm{q}_{j}\left(\mathrm{x}_{\mathrm{i}}=0\right)$ is the normal or resting quantal size at stimulus $\mathrm{j}$ and $\mathrm{q}_{\mathrm{j}}\left(\mathrm{x}_{\mathrm{i}}=1\right)$ is the quantal size at stimulus $j$ after a release event at stimulus $i$, which can be affected by desensitisation or saturation. Now the expectation of the product of the outcome in response to stimulus $i$ and $j$ at different release sites $\mathrm{k}$ and $\mathrm{m}$ in eq. E5 is considered further. Again $\mathrm{q}_{\mathrm{i}}$ is independent from the rest. Release sites are considered independent regarding the release process. The only 
correlation here is between previous release events and the quantal size of the following, e.g. due to neurotransmitter build-up and resulting desensitisation and saturation

$$
<\mathrm{q}_{\mathrm{i}} \mathrm{x}_{\mathrm{ik}} \mathrm{q}_{\mathrm{j}} \mathrm{x}_{\mathrm{jm}}>=\mathrm{q}_{\mathrm{i}}<\mathrm{x}_{\mathrm{ik}} \mathrm{q}_{\mathrm{j}}><\mathrm{x}_{\mathrm{jm}}>
$$

The second expectation term can be expressed in terms of the covariance between the two quantities

$$
<\mathrm{q}_{\mathrm{i}} \mathrm{x}_{\mathrm{ik}} \mathrm{q}_{\mathrm{j}} \mathrm{x}_{\mathrm{jm}}>=\mathrm{q}_{\mathrm{i}}\left(\operatorname{cov}\left(\mathrm{x}_{\mathrm{ik}}, \mathrm{q}_{\mathrm{j}}\right)+<\mathrm{x}_{\mathrm{ik}}>\mathrm{q}_{\mathrm{j}}\right)<\mathrm{x}_{\mathrm{jm}}>
$$

Again for both expectation terms holds $\left\langle\mathrm{x}_{\mathrm{i}}\right\rangle=\mathrm{p}_{\mathrm{Ai}} \mathrm{p}_{0 \mathrm{i}}$ in analogy to the calculation in the methods part

$$
<\mathrm{q}_{\mathrm{i}} \mathrm{x}_{\mathrm{ik}} \mathrm{q}_{\mathrm{j}} \mathrm{x}_{\mathrm{jm}}>=\mathrm{q}_{\mathrm{i}}\left(\operatorname{cov}\left(\mathrm{x}_{\mathrm{ik}}, \mathrm{q}_{\mathrm{j}}\right)+\mathrm{q}_{\mathrm{j}} \mathrm{p}_{\mathrm{Ai}} \mathrm{p}_{0 \mathrm{i}}\right) \mathrm{p}_{\mathrm{Aj}} \mathrm{p}_{0 \mathrm{j}}
$$

Substitution of eq. E7 and E10 into eq. E5 yields

$$
\begin{aligned}
\operatorname{Cov}_{\mathrm{ij}}=\mathrm{Nq}_{\mathrm{i}} \mathrm{q}_{\mathrm{j}}\left(\mathrm{x}_{\mathrm{i}}=1\right) \mathrm{p}_{\mathrm{Ai}} \mathrm{p}_{0 \mathrm{i}} \mathrm{p}_{\mathrm{Aji}} \mathrm{p}_{\mathrm{oj}}+\mathrm{N}(\mathrm{N}-1) \mathrm{q}_{\mathrm{i}} \operatorname{cov}\left(\mathrm{x}_{\mathrm{i}}, \mathrm{q}_{\mathrm{j}}\right) \mathrm{p}_{\mathrm{Aj}} \mathrm{p}_{0 \mathrm{j}} \\
+\mathrm{N}(\mathrm{N}-1) \mathrm{q}_{\mathrm{i}} \mathrm{q}_{\mathrm{j}} \mathrm{p}_{\mathrm{Ai}} \mathrm{p}_{0 \mathrm{i}} \mathrm{p}_{\mathrm{Aj}} \mathrm{p}_{0 \mathrm{j}}-\mathrm{N}^{2} \mathrm{q}_{\mathrm{i}} \mathrm{q}_{\mathrm{j}} \mathrm{p}_{\mathrm{Ai}} \mathrm{p}_{0 \mathrm{i}} \mathrm{p}_{\mathrm{Aj}} \mathrm{p}_{0 \mathrm{j}}
\end{aligned}
$$

With some rearrangement one obtains from this an analogous equation to eq. M11

$$
\operatorname{Cov}_{\mathrm{ij}}=\operatorname{Nq}_{\mathrm{i}} \mathrm{q}_{\mathrm{j}} \mathrm{p}_{\mathrm{Ai}} \mathrm{p}_{0 \mathrm{i}} \mathrm{p}_{0 \mathrm{j}}\left(\frac{\mathrm{q}_{\mathrm{j}}\left(\mathrm{x}_{\mathrm{i}}=1\right)}{\mathrm{q}_{\mathrm{j}}} \mathrm{p}_{\mathrm{Aji}}-\left(1-\frac{(\mathrm{N}-1) \operatorname{cov}\left(\mathrm{x}_{\mathrm{i}}, \mathrm{q}_{\mathrm{j}}\right)}{\mathrm{p}_{\mathrm{Ai}} \mathrm{p}_{0 \mathrm{i}}}\right) \mathrm{p}_{\mathrm{Aj}}\right)
$$

In the case of negligible refilling, i.e. when $\mathrm{p}_{\mathrm{Ai}}=0$, this reduces to an expression in analogy to eq. M16

$$
\operatorname{Cov}_{\mathrm{ij}}=-\mathrm{Nq}_{\mathrm{i}} \mathrm{q}_{\mathrm{j}} \mathrm{p}_{0 \mathrm{i}} \mathrm{p}_{\mathrm{Ai}} \mathrm{p}_{0 \mathrm{j}} \mathrm{p}_{\mathrm{Aj}}\left(1-\frac{(\mathrm{N}-1) \operatorname{cov}\left(\mathrm{x}_{\mathrm{i}}, \mathrm{q}_{\mathrm{j}}\right)}{\mathrm{p}_{\mathrm{Ai}} \mathrm{p}_{0 \mathrm{i}}}\right)
$$

Now defining the new factor as

$$
\mathrm{D}_{\mathrm{ij}}=\left(1-\frac{(\mathrm{N}-1) \operatorname{cov}\left(\mathrm{x}_{\mathrm{i}}, \mathrm{q}_{\mathrm{j}}\right)}{\mathrm{p}_{\mathrm{Ai}} \mathrm{p}_{0 \mathrm{i}}}\right)^{-1}
$$

Information about the new factor $\mathrm{D}_{\mathrm{ij}}$ as compared to eq. M16, can be obtained by the derivation analogous to the one, which led to eq. M17. The division of eq. E13 by E2 and rearrangement yields 


$$
\mathrm{ND}_{\mathrm{ij}}=-\frac{\overline{\mathrm{Y}}_{\mathrm{i}} \overline{\mathrm{Y}}}{\mathrm{CoV}_{\mathrm{ij}}}
$$

This is just the inverse of eq. M17 including the new factor. With eq. E14 it can be tested whether this factor is constant or changes with stimulus number, because $\mathrm{N}$ itself is constant per definition in the model.

Including the new factor into the expressions (eq. M21a, eq. M21c) for calculating the quantal size for each multi-quantal EPSC in the stimulus train from the covariance yields exemplary for eq. M21a

$$
q_{i}=\frac{\operatorname{Var}_{i}}{\bar{Y}_{i}}-D_{i i+1} \frac{\operatorname{Cov}_{i, i+1}}{\bar{Y}_{i+1}}
$$

In case that the factor does not change with stimulus number, which can be tested by evaluating eq. E14, the second term is just scaled with a constant factor here defined as D (for desensitisation factor), such that

$$
\mathrm{q}_{\mathrm{i}}=\frac{\mathrm{Var}_{\mathrm{i}}}{\overline{\mathrm{Y}}_{\mathrm{i}}}-\mathrm{D} \frac{\operatorname{Cov}_{\mathrm{i}, \mathrm{i}+1}}{\mathrm{Y}_{\mathrm{i}+1}}
$$

Thus if D can be determined via eq. E14 or by comparing the calculated initial quantal size $\mathrm{q}_{1}$, which is equivalent to the resting quantal size, to the average mEPSC amplitude if available, the approach for calculating the quantal size for each response in a train from the covariance is still appropriate in the presence of correlation due to e.g. desensitisation and saturation. 


\section{References}

1. Alberts B, Bray D, Lewis J, Raff M, Roberts K, Watson JD (1994) Molecular biology of the cell. $3^{\text {rd }}$ ed. Garland Publishing, New York

2. Ashery U, Koch H, Scheuss V, Brose N, Rettig J (1999) A presynaptic role for the ADP ribosylation factor (Arf)-specific GDP/GTP exchange factor msec7-1. Proc Natl Acad Sci USA, 96: 1094-99

3. Banks MI, Smith PH (1992) Intracellular recording from neurobiotin-labelled celss in brain slices of the rat medial nucleus of the rat trapezoid body. J Neurosci, 12: $2819-37$

4. Barbour B, Häusser M (1997) Intersynaptic diffusion of neurotransmitter. Trends Neurosci, 20(9): 377-84

5. Bekkers JM, Stevens CF (1995) Quantal analysis of EPSCs recorded from small numbers of synapses in hippocampal cultures. J Neurophysiol, 73(3): 1145-56

6. Bekkers JM, Stevens CF (1990) Presynaptic mechanisms for long-term potentiation in the hippocampus. Nature, 346: 724-8

7. Behrends JC, Rumpel E (2000) Inhibitors of vesicle filling selectively affect high frequency transmission at striatal gabanergic synapses in vitro. Europ Biophys J, 29(4-5): 349 (abstract)

8. Borst JGG, Helmchen F, Sakmann B (1995) Pre- and postsynaptic whole-cell recordings in the medial nucleus of the trapezoid body of the rat. $\mathrm{J}$ Physiol, 489(3): $825-40$

9. Borst JGG, Sakmann B (1999) Depletion of calcium in the synaptic cleft of a calyx-type synapse in the rat brainstem. J Physiol, 521(1):123-33

10. Borst JGG, Sakmann B (1998) Facilitation of presynaptic calcium currents in the rat brainstem. J Physiol, 513(1): 149-55

11. Borst JGG, Sakmann B (1996) Calcium influx and transmitter release in a fast CNS synapse. Nature, 383: 431-4

12. Chuma N, Ohmori H (1998) Postnatal development of phase-locked high-fidelity synaptic transmission in the medial nucleus of the trapezoid body of the rat. $\mathrm{J}$ Neurosci, 18(1): 512-20

13. Clamann HP, Mathis J, Lüscher (1989) Variance analysis of excitatory postsynaptic potentials in cat spinal motoneurons during posttetanic potentiation. $\mathbf{J}$ Neurophysiol, 61(2): 403-16 
14. Clements JD, Silver RA (2000) Unveilling synaptic plasticity: A new graphical and analytical approach. TINS, 23(3): 105-13

15. Cuttle MF, Tsujimoto T, Forsythe ID, Takahashi T (1998) Facilitation of the presynaptic calcium current at an auditory synapse in rat. J Physiol, 512(3): 723-9

16. Debanne D, Guerineau NC, Gähwiler BH, Thompson SM (1996) Paired-pulse facilitation and depression at unitary synapses in rat hippocampus: Quantal fluctuation affects subsequent release. J Physiol, 491(1): 163-76

17. Del Castillo J, Katz B (1954) Quantal components of the end-plate potential. J Physiol Lond, 124: 560-73

18. Diamond JS, Jahr CE (1997) Transporters buffer synaptically released glutamate on a submillisecond time scale. J Neurosci, 15: 4672-87

19. Dittmann JS, Kreitzer AC, Regehr WG (2000) Interplay between facilitation, depression, and residual calcium at three presynaptic terminals. J Neurosci. 20(4): 1374-85

20. Dobrunz LE, Huang EP, Stevens CF (1997) Very short-term plasticity in hippocampal synapses. Proc Natl Acad Sci USA, 94: 14843-47

21. Edwards FA, Konnerth A, Sakmann B (1990) Quantal analysis of inhibitory synaptic transmission in the dentate gyrus of rat hippocampal slices: A patch clamp study. J Physiol, 430: 213-49

22. Edwards FA, Konnerth A, Sakmann B, Takahashi T (1989) A thin slice preparation for patch clamp recordings from neurones of the mammalian central nervous system. Pflügers Arch, 414: 600-12

23. Elmquist D, Quastel DMJ (1964) A quantitative study of the end-plate potentials in isolated human muscle. J Physiol, 178: 505-29

24. Faber DS, Waldeck RF, Pereda A (1998) Synaptic depression at an identified central synapse: Evidence for different constraints on evoked and spontaneous quanta. In: Central synapses: Quantal mechanisms and plasticity (Faber DS, Redman SJ, Thompson SM, Altman JS, eds): Strasbourg, France: HFSP: 158-68

25. Faber DS, Korn H (1991) Applicability of the coefficient of variation method for analysing synaptic plasticity. Biophys J, 60: 1288-94

26. Forsythe ID, Tsujimoto T, Barnes-Davies M, Cuttle MF, Takahashi T (1998) Inactivation of presynaptic calcium current contributes to synaptic depression at a fast central synapse. Neuron, 20(4): 797-807

27. Forsythe ID, Barnes-Davies M, Brew HM (1995) The calyx of Held: A models for transmission at mammalian glutamatergic synapses. In: Excitatory amino acids and synaptic transmission, $2^{\text {nd }}$ ed. Wheal H, Thomson A (Eds). Academic Press Inc, San Diego 
28. Forsythe ID, Barnes-Davies M (1993) The binaural auditory pathway: Excitatory amino acid receptors mediate dual time course excitatory postsynaptic currents in the rat medial nucleus of the trapezoid body. Proc R Soc Lond [Biol], 251: 151-7

29. Forti L, Bossi M, Bergamaschi A, Villa A, Malgaroli A (1997) Loose-patch recordings of single quanta at individual hippocampal synapses. Nature, 388: 8748

30. Foster M, Sherrington CS (1897) A textbook of Physiology, Part III: The central nervous system. $7^{\text {th }}$ ed, Macmillan, London

31. Frerking M, Wilson M (1996) Effects of variance in miniamplitude on stimulusevoked release: A comparison of two models. Biophys J, 70: 2078-91

32. Garner CC, Kindler S, Gundelfinger ED (2000) Molecular determinants of presynaptic active zones. Curr Opinion Neurobiol, 10: 321-7

33. Geiger JRP, Melcher T, Koh DS, Sakmann B, Seeburg PH, Jonas P, Monyer H (1995) Relative abundance of subunit mRNAs determines gating and $\mathrm{Ca}^{2+}$ permeability of AMPA receptors in principal neurons and interneurons in rat CNS. Neuron, 15: 193-204

34. Gummer AW, Zenner HP (1996) Central processing of auditory information. In: Comprehensive human physiology, 1. Greger R, Windhorst U (Eds). SpringerVerlag Berlin Heidelberg

35. Heineman SF, Conti F (1992) Non stationary noise analysis and application to patch clamp recordings. Methods Enzymol, 207: 131-148

36. Held H (1893) Die centrale Gehörleitung. Arch Anat Physiol Anat Abtheil, 17: 201-248

37. Helmchen F, Borst JGG, Sakmann B (1997) Calcium dynamics associated with a single action potential in a CNS presynaptic terminal. Biophys J. 72: 1458-71

38. Hsu SF, Augustine GJ, Jackson MB (1996) Adaption of $\mathrm{Ca}^{2+}$-triggered exocytosis in presynaptic terminals. Neuron, 17: 501-512

39. Hurlbut WP, Iezzi N, Fesce R, Ceccarelli B (1990) Correlation between quantal secretion and vesicle loss at the frog neuromuscular junction. J Physiol, 425: 50126

40. Kasai $\mathrm{H}$ (1993) Cytosolic $\mathrm{Ca}^{2+}$ gradients, $\mathrm{Ca}^{2+}$ binding proteins and synaptic plasticity. Neurosci Res, 16: 1-7

41. Korn H, Sur C, Charpier S, Legendre P, Faber DS (1994) The one-vesicle hypothesis and multivesicular release. In: Molecular and cellular mechanisms of neurotransmitter release. Eds: Stjärne L, Greengard P, Grillner S, Hökfeld T, Ottoson D. Raven Press, New York 
42. Korn H, Faber DS (1991) Quantal analysis and synaptic efficacy in the CNS. Trends Neurosci, 14(10): 439-45

43. Korn H, Mallet A, Triller A, Faber DS (1982) Transmission at a central inhibitory synapse: II. Quantal description of release, with a physical correlate for the binomial n. J Neurophysiol, 48: 679-707

44. Kraushaar U, Jonas P (2000) Efficacy and Stability of Quantal GABA release at a hippocampal interneuron-principal neuron synapse. J Neurosci, 20(15): 55945607

45. Liu G, Tsien RW (1995) Properties of synaptic transmission at single hippocampal synaptic boutons. Nature, 375: 404-8

46. Malinow R, Tsien RW (1990) Presynaptic enhancement shown by whole-cell recordings of long-term potentiation in hippocampal slices. Nature, 346: 177-180

47. Marty A, Neher E (1995) Tight-seal whole-cell recording. In: Single-channel recording. Eds: Neher E, Sakmann B. Plenum Press, $2^{\text {nd }}$ ed

48. Matveev V, Wang XJ (2000) Implications of all-or-none synaptic transmission and short-term synaptic depression beyond vesicle depletion: A computational study. J Neurosci, 20(4): 1575-88

49. McLachlan EM (1978) The statistics of transmitter release at chemical synapses. Int Rev Physiol, Neurophysiol III, 17: 49-117

50. Meyer A (1999) Untersuchungen zur Wahrscheinlichkeit der Transmitterfreisetzung an eine zentralen Synapse. Graduate Diploma Thesis, University of Göttingen, Germany

51. Murthy VN, Sejnowski TJ, Stevens CF (1997) Heterogeneous release properties of visualized individual hippocampal synapses. Neuron 18: 599-612

52. Neher E, Sakaba T (2000) Combining deconvolution and noise analysis for the estimation of transmitter release rates at the calyx of Held. J Neurosci, submitted

53. Neher E (1998) Vesicle pools and $\mathrm{Ca}^{2+}$ domains: New tools for understanding their roles in neurotransmitter release. Neuron, 20: 389-99

54. Oleskevich S, Clements J, Walmsley B (2000) Release probability modulates short-term plasticity at a rat giant terminal. J Physiol, 524(2): 513-23

55. Orear J (1982) Least squares when both variables have uncertainties. Am J Physics. 50(10): 912-6

56. Otis T, Zhang Su, Trussel LO (1996a) Direct measurement of AMPA receptor desensitization induced by glutamatergic synaptic transmission. J Neurosci, 16(23): 7496-504 
57. Otis TS, Wu, YC, Trussel LO (1996b) Delayed clearance of transmitter an the role of glutamate transporters at synapses with multiple release sites. J Neurosci, 16(5): 1634-44

58. Partin KM, Patneau DK, Mayer ML (1994) Cyclothiazide differentially modulates desensitization of a-amino-3-hydroxy-5-methyl-4-isoxazoleproprionic acid receptor splice variants. Mol Pharmacol. 46: 129-38

59. Quastel DMJ (1997) The binomial model in fluctuation analysis of quantal neurotransmitter release. Biophys J, 72: 728-53

60. Raman IM, Trussel LO (1992) The kinetics of the response to glutamate and kainate in neurons of the avian cochlear nucleus. Neuron, 9: 173-86

61. Redman S (1990) Quantal analysis of synaptic potentials in neurons of the central nervous system. Physiol Rev, 20(1): 165-98

62. Reid CA, Clements JD (1999) Postsynaptic expression of long-term potentiation in the rat dentate gyrus demonstrated by variance-mean analysis. J Physiol, 518(1): $121-30$

63. Reyes A, Lujan R, Rozov A, Burnashev N, Somogyi P, Sakmann B (1998) Target cell-specific-specific facilitation and depression in neocortical circuits. Nature Neurosci, 1(4): 279-85

64. Rosenmund C, Clements JD, Westbrook GL (1993) Nonuniform probability of glutamate release at a hippocampal synapse. Science, 262: 754-57

65. Sakaba T, Neher E (2000) Quantitative relationship between transmitter release and calcium current at the calyx of Held synapse. J Neurosci, submitted

66. Schikorski T, Stevens CF (1997) Quantitative ultrastructural analysis of excitatory hippocampal synapses. J Neurosci, 17(15): 5858-67

67. Schneggenburger R, Neher E (2000) Intracellular calcium dependence of transmitter release rates at a fast central synapse. Nature, 406: 889-93

68. Schneggenburger R, Meyer A, Neher E (1999) Released fraction and total size of a pool of immediately available transmitter quanta at a calyx synapse. Neuron, 23: 399-409

69. Sigworth FJ (1980) The variance of sodium current fluctuations at the node of Ranvier. J Physiol, 307: 97-129

70. Silver RA, Momiyama A, Cull-Candy S (1998) Locus of frequency-dependent depression identified with multiple-probability fluctuation analysis at rat climbing fibre-Purkinje cell synapses. J Physiol, 510(3): 881-902 
71. Stevens CF, Tsujimoto T (1995) Estimates of the pool size of releasable quanta at a single central synapse and for the time required to refill the pool. Proc Natl Acad Sci USA, 92: 846-49

72. Südhof TC (1995) The synaptic vesicle cycle: A cascade of protein-protein interactions. Nature, 375: 645-53

73. Takahashi T, Forsythe ID, Tsujimoto T, Barnes-Davies M, Onodera K (1996) Presynaptic calcium current modulation by metabotropic glutamate receptors. Science, 274(5287): 594-7

74. Thompson SM, Debanne D, Capogna M (1998) Presynaptic determinants of synaptic efficacy in hippocampal pyramidal neurons. In: Central synapses: Quantal mechanisms and plasticity (Faber DS, Redman SJ, Thompson SM, Altman JS, eds): Strasbourg, France: HFSP: 247-54

75. Tong G, Jahr CE (1994) Multivesicular release from excitatory synapses of cultured hippocampal neurons. Neuron, 12: 51-59

76. Traynelis SF (1998) Software-based correction of single compartment series resistance errors. J Neurosci Methods, 86: 25-34

77. Trommershäuser J (2000) A semi-microscopic model of synaptic transmission and plasticity. PhD-thesis. Georg-August-University, Göttingen, Germany

78. Trussel LO, Zhang S, Raman IM (1993) Desensitization of AMPA receptors upon multiquantal neurotransmitter release. Neuron, 10: 1185-1196

79. Vere-Jones D (1966) Simple stochastic models for the release of quanta of transmitter from a nerve terminal. Austr J Statistics, 8(2): 53-63

80. Voets T, Neher E, Moser T (1999) Mechanisms underlying phasic and sustained secretion in chromaffin cells from mouse adrenal slices. Neuron, 23: 607-15

81. von Gersdorff H, Schneggenburger R, Weis S, Neher E (1997) Presynaptic depression at a calyx synapse: The small contribution of metabotropic glutamate receptors. J Neurosci, 17(21): 8137-46

82. von Kitzing E, Jonas P, Sakmann B (1994) Quantal analysis of excitatory postsynaptic currents at the hippocampal mossy fiber-CA3 pyramidal cell synapse. In: Molecular and cellular mechanisms of neurotransmitter release. Eds: Stjärne L, Greengard P, Grillner S, Hökfeld T, Ottoson D. Raven Press, New York

83. Waldeck RF, Pereda A, Faber DS (2000) Properties and plasticity of paired-pulse depression at a central synapse. J Neurosci, 20(14): 5312-5320

84. Walmsley B, Alvarez FJ, Fyffe REW (1998) Diversity of structure and function at mammalian central synapses. TINS, 21(2): 81-8 
85. Walmsley B (1993) Quantal analysis of synaptic transmission. In Electrophysiology. Wallis DI (ed.)

86. Wang LY, Kaczmarek LK (1998) High-frequency firing helps replenish the readily releasable pool of synaptic vesicles. Nature, 394: 384-7

87. Weis S, Scheggenburger R, Neher E (1999) Properties of a model of $\mathrm{Ca}^{2+}$ dependent vesicle pool dynamics and short term synaptic depression. Biophys $\mathrm{J}$, 77: 2418-29

88. Wernig A (1974) Estimates of the statistical release parameters from crayfish and frog neuromuscular junctions. J Physiol, 244: 207-21

89. Wu LG, Borst JG (1999) The reduced release probability of releasable vesicles during recovery from short-term synaptic depression. Neuron, 23: 821-32

90. Wu LG, Saggau P (1997) Presynaptic inhibition of elicited neurotransmitter release. Trends Neurosci, 20(5): 204-212

91. Xu T, Binz T, Niemann H, Neher E (1998) Multiple kinetic components of exocytosis distinguished by neurotoxin sensitivity. Nature Neurosci, 1(3): 192-200

92. Yamada KA, Tang CM (1993) Benzothiadiazides inhibit rapid glutamate receptor desensitization and enhance glutamatergic synaptic currents. J Neurosci,

93. Yamada KA, Rothman SM (1992) Diazoxide blocks glutamate receptor desensitization and prolongs excitatory currents in rat hippocampal neurones. $\mathrm{J}$ Physiol, 458: 409-23

94. Zucker RS (1999) Calcium- and activity-dependent synaptic plasticity. Curr Opin Neurobiol, 9(3): 305-13

95. Zucker RS (1989) Short-term synaptic plasticity. Ann Rev Neurosci. 12: 13-31

96. Zucker RS (1973) Changes in the statistics of transmitter release during facilitation. J Physiol, 229: 787-810 


\section{Acknowledgements}

First of all, I thank my supervisor Prof. Erwin Neher for giving me the opportunity to work on synaptic transmission in his laboratory and for his constant input and guidance during the course of the project.

I thank my advisor Dr. habil. Jens Rettig for his generous support, for teaching me electrophysiology on the nmj preparation, and for giving me the biochemistry and molecular biology background knowledge necessary for our work. Furthermore I like to thank him for his understanding and the uncomplicated solution, when the $\mathrm{nmj}$ preparation was discarded, that I could continue the $\mathrm{PhD}$ project on the calyx of Held synapse, but still could work with him on new projects in the hippocampal cell culture.

I'm indebted to my advisor Dr. Ralf Schneggenburger, that he took me on to his group for continuing the $\mathrm{PhD}$ project on the calyx of Held synapse. Furthermore I thank him for introducing me to electrophysiological recordings in brain slices, and his kind support.

Finally I like to thank my family and friends for their unfailing kindness, understanding, and encouragement. 


\section{Publications}

1. Ashery U, Koch H, Scheuss V, Brose N, Rettig J (1999) A presynaptic role for the ADP ribosylation factor (ARF)-specific GDP / GTP exchange factor msec7-1. Proc Natl Acad Sci USA, 96: 1094-9

2. Lao G, Scheuss V, Gerwin CM, Su Q, Mochida S, Rettig J, Sheng ZH (2000) Syntaphilin: A synataxin-1 clamp that controls SNARE assembly. Neuron, 25: 191-201

3. Scheuss V, Schneggenburger R, Neher E (2000) Covariance analysis of EPSCs during synaptic depression reveals a reduction of quantal size. Eurobiophysics 2000 supplement, abstract 6B-7, $3^{\text {rd }}$ European Biophysics Congress, München 


\section{Curriculum Vitae}

\section{Personal Data}

Date of Birth 17.2.72

Location of Birth Hamburg, Germany

Nationality German

Studies and Training

1991

Abitur in Physics, Mathematics, Latin and History

Schulzentrum Bördestraße, Bremen, Germany

$1994 \quad$ Vordiplom in Physics

University of Bremen, Germany

1996 Bachelor of Science

in "Applied Physics with Microelectronics and Computing",

Kingston University, Kingston, England

„Oxford Scientific Products Prize“ for overall performance

„Tangye Fluid Mechanics Prize“ for achievements in fluid mechanics

1997

Master of Science

in "Engineering and physical science in medicine"

Imperial College, London, England

Scholar of the Dr.Carl-Duisberg-Stiftung

since 1998 PhD student

Max-Planck-Institut für Biophysikalische Chemie, Göttingen,

Germany

Scholar of the Graduate College

"Signal-mediated transport of proteins and vesicles"

since 1999 Scholar and Speaker of the Graduate College

"Protein-Protein Interactions during Intracellular Transport of Macromolecules" 